
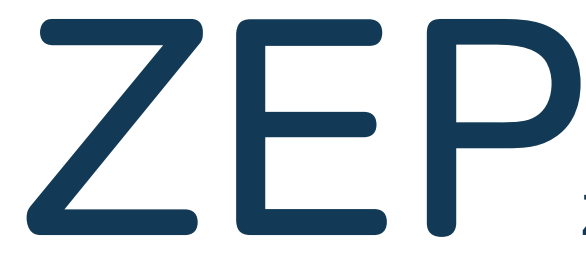

Zeitschrift für internationale Bildungsforschung und Entwicklungspädagogik

Mit: Mitteilungen der DGfE-Kommission

Vergleichende und Internationale

Erziehungswissenschaft

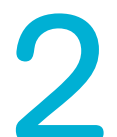

Sustainable • Die Bedeutung von BNE für das Erreichen der Sustainable Development

Development Goals (SDGs)

Goals • Die Bildungsagenda der Vereinten Nationen aus dem Blick der internationalen Bildungsforschung

- Die Umsetzung der SDGs im deutschen Schulsystem

- Teaching the Sustainable Development Goals - Geschichten des Wandels

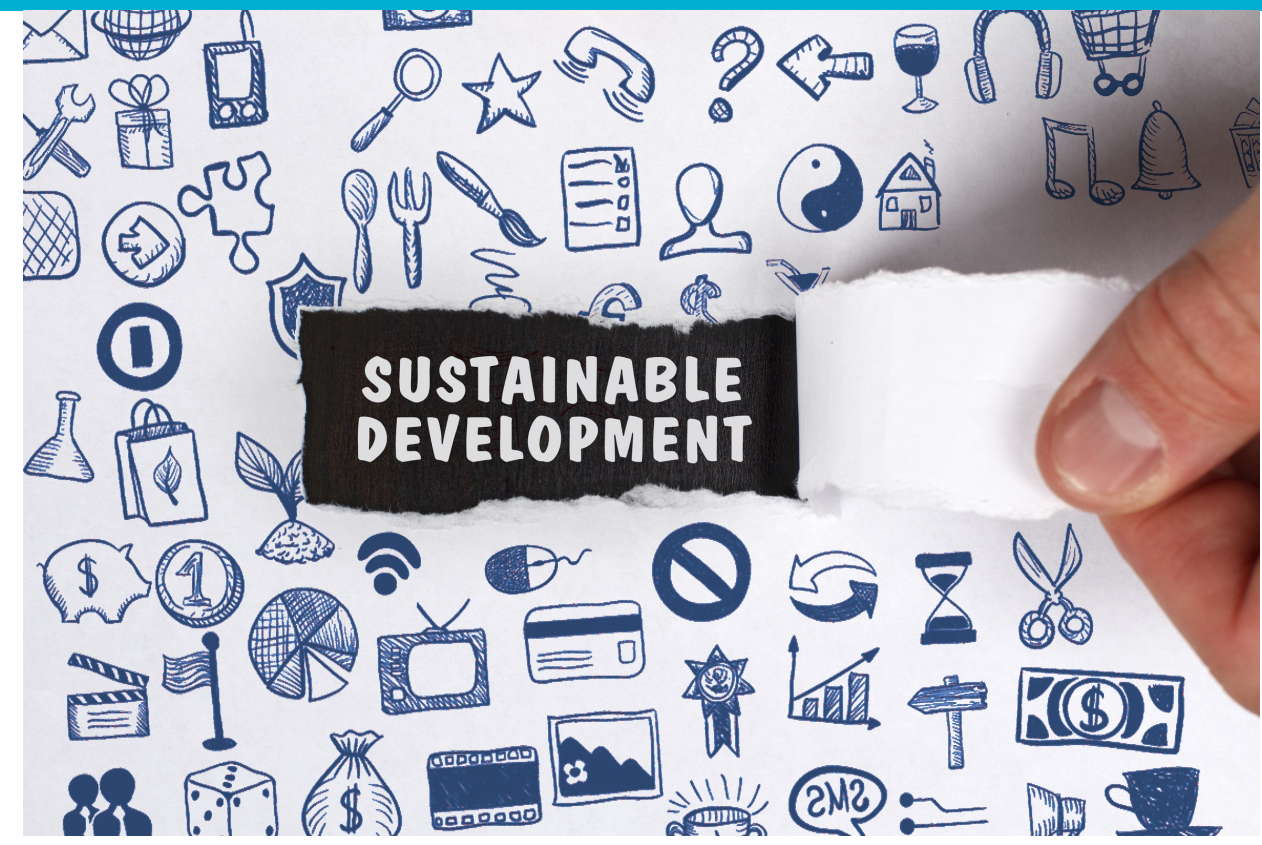


A m 25. September 2015 verabschiedete die UN-Vollversammlung die Agenda 2030 für Nachhaltige Entwicklung mit 17 Nachhaltigkeitszielen (Sustainable Development Goals - SDGs). Diese Ziele beschreiben wichtige Entwicklungsherausforderungen, um für alle jetzt und in Zukunft ein nachhaltiges, friedliches, wohlhabendes und gerechtes Leben zu sichern. Ein wesentliches Merkmal der Agenda 2030 ist ihre Unteilbarkeit und Universalität. Sie richtet sich gleichermaßen an den Globalen Süden und Norden.

Im Ziel 4.7 des SDG 4 zu hochwertiger Bildung werden Bildung für nachhaltige Entwicklung (BNE) und Global Citizenship Education (GCED) ausdrücklich als relevante Bildungskonzepte anerkannt. Gleichzeitig gilt es, die zentrale Bedeutung von BNE für alle anderen 16 SDGs zu betonen: BNE soll allen Personen ermöglichen, zur Erreichung der SDGs beizutragen, indem sie die notwendigen Kenntnisse über (nicht-)nachhaltige Entwicklungen und Kompetenzen erwerben können, die sie nicht nur brauchen, um zu verstehen, worum es bei den SDGs geht, sondern auch, um sich als informierte Bürger/innen aktiv für die Herbeiführung der notwendigen gesellschaftlichen Transformation einzusetzen. Es geht dabei nicht nur allein darum, auf die existenten Problemlagen reagieren zu können, sondern vor allem darum, vorausschauend mit Zukunft umzugehen sowie an innovativen Lebens- und Gesellschaftsentwürfen mitzuwirken, die einen zukunftsweisenden und verantwortlichen Übergang in eine nachhaltige Welt möglich machen.

\section{Impressum}

ZEP - Zeitschrift für internationale Bildungsforschung und Entwicklungspädagogik ISSN 1434-4688

\section{Herausgeber:}

Gesellschaft für interkulturelle Bildungsforschung und Entwicklungspädagogik e.V. und KommEnt

Schriftleitung: Annette Scheunpflug/

Claudia Bergmüller

\section{Technische Redaktion:}

Claudia Bergmüller (verantwortlich) 0951/863-

1832, Markus Ziebarth (Rezensionen),

Anne-Christine Banze (Schlaglichter)

\section{Redaktionsanschrift:}

ZEP-Redaktion, Lehrstuhl Allgemeine

Pädagogik, Markusplatz 3, 96047 Bamberg

\section{Verlag:}

Waxmann Verlag GmbH, Steinfurter Straße 555 48159 Münster, Tel.: 0251/26 5040

E-Mail:info@waxmann.com
Zielsetzung dieses Themenheftes ist es, eine Einführung in die SDGs, insbesondere das Bildungs-SDG, und die Bedeutung von Bildungs- und Lernprozessen für die SDGs zu geben. Zudem wird beleuchtet, inwiefern in Deutschland die SDGs bereits Eingang in das Bildungssystem gefunden haben.

Marco Rieckmann befasst sich mit der Bedeutung von BNE für das Erreichen der SDGs. Dabei wird deutlich, dass BNE die Entwicklung von Nachhaltigkeitskompetenzen fördern kann, die Individuen dazu befähigen, an der Umsetzung der SDGs mitzuwirken. Gleichzeitig kann BNE auch spezifische Lernprozesse ermöglichen, die dazu benötigt werden, sich ein bestimmtes SDG zu erschließen und eigene Beiträge zu diesem Ziel zu leisten.

Christel Adick befasst sich mit der Bildungsagenda der Vereinten Nationen bis zum Jahr 2030 aus dem Blickwinkel der internationalen Bildungsforschung. Zunächst werden die SDGs mit anderen UN-Programmen seit den 1990er Jahren historisch kontextualisiert, um dadurch die Bedeutsamkeit des SDG 4 besser einordnen zu können. Zudem werden die zehn Unterpunkte des SDG 4 hinsichtlich ihrer Aussagen zu den angestrebten Entwicklungen analysiert. Beleuchtet wird außerdem die inzwischen im System der Vereinten Nationen begonnene Arbeit an Indikatoren, mit denen die Realisierung des SDG 4 jährlich weltweit erhoben und verglichen werden soll. Abschließend wird die Anschlussfähigkeit des SDG 4-Monitoring an Forschung und Lehre im Rahmen einer internationalen Bildungsforschung reflektiert.
Georg Müller-Christ, Bror Giesenbauer und Merle Katrin Tegeler stellen Ergebnisse einer Studie im Auftrag des Rats für nachhaltige Entwicklung der Bundesregierung zur Umsetzung der SDGs im deutschen Bildungssystem vor. Auf Basis von Experteninterviews und Online-Recherchen werden sechs Bildungsbereiche einzeln beleuchtet: Frühkindliche Bildung, Schule, berufliche Bildung, Hochschule, non-formales und informelles Lernen sowie Fort- und Weiterbildung. Es zeigt sich, dass die SDGs bislang v.a. dort transportiert werden, wo Umweltbildung, Globales Lernen oder BNE bereits Pfade angelegt haben. Gleichwohl scheint der Ordnungsrahmen der SDGs den bereits Aktiven Schwung zu geben, an der Umsetzung einer nachhaltigen Entwicklung weiterzuarbeiten und ihre Aktivitäten ansprechend darzustellen.

Das Lehrmaterial „Teaching the Sustainable Development Goals" stellt Thomas Hoffmann vor. Es ist 2017 im Rahmen des interkulturell konzipierten ESD Expert Net Mexico, India, South Africa and Germany entstanden. Eine Besonderheit des Materials ist, dass hier positive Erzählungen des Wandels genutzt werden, um zu zeigen, dass die SDGs erreicht werden können.

Zudem erhalten Sie auch in dieser Ausgabe Informationen zum Globalen Lernen und zur internationalen Bildungsforschung etwa in Form von Rezensionen und Veranstaltungshinweisen.

Eine anregende Lektüre wünschen Achim Beule und Marco Rieckmann Stuttgart/Vechta, Juli 2018 doi.org/10.31244/zep.2018.02.01

Begründet von: Alfred K. Treml (†) mit dem AK Dritte Welt Reutlingen.

Ehemals in der Redaktion: Barbara Asbrand, Hans Gängler, Sigrid Görgens, Richard Helbling (Schweiz), Linda Helfrich, Alfred Holzbrecher, Torsten Jäger, Gerhard Mersch, Renate Nestvogel, Gottfried Orth, Georg Friedrich Pfäfflin, Arno Schöppe, Birgit Schößwender, Horst Siebert, Klaus-Jürgen Tillmann, Barbara Toepfer, Erwin Wagner, Joachim Winter.

Aktuell in der Redaktion: Achim Beule, Claudia Bergmüller, Hans Bühler, Christian Brüggemann, Asit Datta, Julia Franz, Norbert Frieters-Reermann, Heidi Grobbauer (Österreich), Helmuth Hartmeyer (Österreich), Susanne Höck, Karola Hoffmann, Lydia Kater-Wettstädt, Ulrich Klemm, Gregor LangWojtasik, Sarah Lange, Volker Lenhart, Claudia Lohrenscheit, Bernd Overwien, Marco Rieckmann, Annette Scheunpflug, Klaus Seitz, Susanne Timm, Rudolf Tippelt

Anzeigenverwaltung: Waxmann Verlag GmbH, Maike Rösmann: roesmann@waxmann.com

Abbildungen: (Falls nicht bezeichnet) Privatfotos oder Illustrationen der Autor/inn/en

Titelbild: (C)Egor, www.stock.adobe.com

Erscheinungsweise und Bezugsbedingungen: erscheint vierteljährlich; Jahresabonnement EUR 24,00, Einzelheft EUR 11,00; alle Preise verstehen sich zuzüglich Versandkosten; zu beziehen durch alle Buchhandlungen oder direkt vom Verlag. Abbestellungen spätestens acht Wochen vor Ablauf des Jahres. Das Heft ist auf umweltfreundlichem chlorfreien Papier gedruckt. Diese Publikation ist gefördert von Brot für die Welt - Evangelischen Entwicklungsdienst, Referat für Inlandsförderung, Berlin. 
Zeitschrift für internationale Bildungsforschung und Entwicklungspädagogik

Mit: Mitteilungen der DGfE-Kommission

Vergleichende und Internationale Erziehungswissenschaft

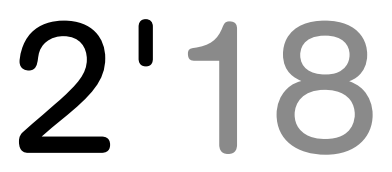

\section{Marco Rieckmann}

Themen 4 Die Bedeutung von Bildung für nachhaltige Entwicklung für das Erreichen der Sustainable Development Goals (SDGs)

Christel Adick

11 Die Bildungsagenda der Vereinten Nationen aus dem

Blickwinkel der internationalen Bildungsforschung

Georg Müller-Christ / Bror Giesenbauer / Merle Katrin Tegeler

19 Die Umsetzung der SDGs im deutschen Bildungssystem Studie im Auftrag des Rats für nachhaltige Entwicklung der Bundesregierung

\section{Thomas Hoffmann}

27 Teaching the Sustainable Development Goals Geschichten des Wandels

Porträt 35 Zwei Angebote für einen besseren Materialüberblick

VENRO 37 BMZ-Wirkungsstudie in der Inlandsarbeit und VENRO-Begleitprojekt

VIE 38 Faire Schule meets SDG-Labor/Hochwertige Bildung für Alle/ GloNet - das Netzwerk für Globales Lernen in der beruflichen Bildung

41 Rezensionen

43 Schlaglichter 


\section{Die Bedeutung von Bildung für nachhaltige Entwicklung für das Erreichen der Sustainable Development Goals (SDGs)}

\begin{abstract}
Zusammenfassung
Die 2015 von den Vereinten Nationen verabschiedeten Sustainable Development Goals (SDGs) zielen darauf, für alle, jetzt und in Zukunft, ein nachhaltiges, friedliches, wohlhabendes und gerechtes Leben zu ermöglichen. Für das Erreichen der SDGs kommt Bildung für nachhaltige Entwicklung (BNE) eine wichtige Bedeutung zu. BNE kann die Entwicklung von Nachhaltigkeitskompetenzen fördern, die Individuen nicht nur dazu befähigen, die SDGs in ihrer ganzen Komplexität zu verstehen, sondern auch eigene Beiträge zum Erreichen der Ziele zu leisten. Gleichzeitig kann BNE auch spezifische Lernprozesse ermöglichen, die dazu benötigt werden, sich ein bestimmtes SDG zu erschließen und eigene Beiträge zu diesem Ziel zu leisten. Dazu bietet der von der UNESCO veröffentliche Leitfaden „Education for Sustainable Development Goals. Learning Objectives“ vielfältige Anregungen. Für die Entwicklung der Nachhaltigkeitskompetenzen und eine Auseinandersetzung mit den einzelnen SDGs bedarf es einer handlungsorientierten, transformativen Pädagogik.
\end{abstract}

Schlüsselworte: Bildung für nachhaltige Entwicklung, Sustainable Development Goals (SDGs), transformative Pädagogik

\footnotetext{
Abstract

The Sustainable Development Goals (SDGs), adopted by the United Nations in 2015, aim to provide a sustainable, peaceful, prosperous and just life for all, now and in the future. Education for Sustainable Development (ESD) plays an important role in achieving the SDGs. ESD can foster the development of sustainability competencies that not only empower individuals to understand the full complexity of the SDGs, but also to make their own contribution to achieving the goals. At the same time, ESD can also facilitate specific learning outcomes needed to understand a specific SDG and to contribute to achieving that goal. The guide "Education for Sustainable Development Goals. Learning Objectives", published by the UNESCO, offers diverse suggestions for such learning outcomes and how to foster them. For the development of the sustainability competencies and
}

learning outcomes related to specific SDGs, an action-oriented, transformative pedagogy is needed.

Keywords: Education for sustainable development, Sustainable Development Goals (SDGs), transformative pedagogy

\section{Die Nachhaltigkeitsziele - eine ambitionierte und universelle Agenda für die Transformation der Weltgesellschaft}

Am 25. September 2015 verabschiedete die UN-Vollversammlung die Agenda 2030 für Nachhaltige Entwicklung (Vereinte Nationen, 2015). Dieses neue globale Rahmenwerk zur nachhaltigen Entwicklung der Weltgesellschaft wurde im Anschluss an die UN-Konferenz über nachhaltige Entwicklung (Rio + 20) in Rio de Janeiro (Brasilien) im Juni 2012 in einem dreijährigen Prozess entwickelt, an dem neben den UN-Mitgliedstaaten, NGOs und anderen Akteuren auch Millionen von Menschen aus der ganzen Welt beteiligt waren (Kercher, 2015; Scholz, 2015, 2017). Im Mittelpunkt der Agenda 2030 stehen 17 Nachhaltigkeitsziele (Sustainable Development Goals, SDGs).

Die universellen, transformativen und inklusiven SDGs beschreiben wichtige Entwicklungsherausforderungen für die Menschheit. Das Ziel der 17 SDGs (Abb. 1) ist es, für alle, jetzt und in Zukunft, ein nachhaltiges, friedliches, wohlhabendes und gerechtes Leben zu ermöglichen. Die SDGs beziehen sich auf globale Herausforderungen, die für das Überleben der Menschheit entscheidend sind. Sie setzen ökologische Grenzen fest und definieren kritische Schwellenwerte für den Einsatz natürlicher Ressourcen. Sie erkennen an, dass Armutsbekämpfung Hand in Hand mit Strategien gehen muss, die wirtschaftliche Entwicklung befördern. Sie widmen sich einer Reihe von sozialen Bedürfnissen, einschließlich Bildung, Gesundheit, sozialer Absicherung und Beschäftigungsmöglichkeiten, während sie gleichzeitig auf die Bewältigung des Klimawandels und Umweltschutz abzielen. Die SDGs adressieren die wichtigsten systemischen Barrieren für eine nachhaltige Entwicklung wie Ungleichheit, nicht-nachhaltige Konsummuster, schwache institutionelle Kapazitäten und Umweltzerstörung (Messner \& Scholz, 2015; Scholz, 2015, 2017). 
1. Keine Armut - Armut in jeder Form und überall beenden

2. Keine Hungersnot - Den Hunger beenden, Ernährungssicherheit und eine bessere Ernährung erreichen und eine nachhaltige Landwirtschaft fördern

3. Gute Gesundheitsversorgung und Wohlergehen - Ein gesundes Leben für alle Menschen jeden Alters gewährleisten und ihr Wohlergehen fördern

4. Hochwertige Bildung - Inklusive, gerechte und hochwertige Bildung gewährleisten und Möglichkeiten des lebenslangen Lernens für alle fördern

5. Gleichberechtigung der Geschlechter - Geschlechtergerechtigkeit und Selbstbestimmung für alle Frauen und Mädchen erreichen

6. Sauberes Wasser und sanitäre Einrichtungen - Verfügbarkeit und nachhaltige Bewirtschaftung von Wasser und Sanitärversorgung für alle gewährleisten

7. Erschwingliche und saubere Energie - Zugang zu bezahlbarer, verlässlicher, nachhaltiger und zeitgemäßer Energie für alle sichern

8. Gute Arbeitsplätze und wirtschaftliches Wachstum - Dauerhaftes, inklusives und nachhaltiges Wirtschaftswachstum, produktive Vollbeschäftigung und menschenwürdige Arbeit für alle fördern

9. Industrie, Innovation und Infrastruktur - Eine belastbare Infrastruktur aufbauen, inklusive und nachhaltige Industrialisierung fördern und Innovationen unterstützen

10. Reduzierte Ungleichheiten - Ungleichheit innerhalb von und zwischen Staaten verringern

11. Nachhaltige Städte und Gemeinden - Städte und Siedlungen inklusiv, sicher, widerstandsfähig und nachhaltig machen

12. Verantwortungsvoller Konsum und Produktion - Für nachhaltige Konsum- und Produktionsmuster sorgen

13. Klimaschutz - Umgehend Maßnahmen zur Bekämpfung des Klimawandels und seiner Auswirkungen ergreifen

14. Leben im Wasser - Ozeane, Meere und Meeresressourcen im Sinne einer nachhaltigen Entwicklung erhalten und nachhaltig nutzen

15. Leben an Land - Landökosysteme schützen, wiederherstellen und ihre nachhaltige Nutzung fördern, Wälder nachhaltig bewirtschaften, Wüstenbildung bekämpfen, Bodenverschlechterung stoppen und umkehren und den Biodiversitätsverlust stoppen

16. Frieden, Gerechtigkeit und starke Institutionen - Friedliche und inklusive Gesellschaften im Sinne einer nachhaltigen Entwicklung fördern, allen Menschen Zugang zur Justiz ermöglichen und effektive, rechenschaftspflichtige und inklusive Institutionen auf allen Ebenen aufbauen

17. Partnerschaften für die Ziele - Umsetzungsmittel stärken und die globale Partnerschaft für nachhaltige Entwicklung wiederbeleben

Quelle: http://www.bmz.delde/ministerium/ziele/2030_agenda/17_ziele/index.html

Damit die Ziele erreicht werden können, müssen viele ihren Beitrag leisten: Regierungen, die Privatwirtschaft, die Zivilgesellschaft und alle Menschen auf der ganzen Welt. Es wird von den Regierungen erwartet, dass sie sich die Ziele zu eigen machen und nationale Rahmenbedingungen sowie politische Strategien und Maßnahmen zur Umsetzung der Agenda 2030 schaffen.

Ein wesentliches Merkmal der Agenda 2030 ist ihre Universalität und Unteilbarkeit. Sie richtet sich an alle Länder - aus dem
Globalen Süden und dem Globalen Norden. Alle Länder, die sich der Agenda 2030 verschreiben, müssen ihre eigenen Entwicklungsbemühungen an dem Ziel ausrichten, den Wohlstand zu fördern und gleichzeitig den Planeten zu schützen, um eine nachhaltige Entwicklung zu erreichen (Messner \& Scholz, 2015). Insofern können in Bezug auf die SDGs alle Länder als Entwicklungsländer betrachtet werden, und alle Länder müssen dringende Maßnahmen zur Förderung einer nachhaltigen Entwicklung ergreifen.

\section{Bildung für nachhaltige Entwicklung - ein Schlüsselinstrument für das Erreichen der Nachhaltigkeitsziele}

\author{
"Education can, and must, contribute to a new vision of \\ sustainable global development." (UNESCO, 2015b, S. 32)
}

Den Weg einer nachhaltigen Entwicklung einzuschlagen, wird eine tiefgreifende Transformation unserer Art zu Denken und zu Handeln erfordern. Um eine nachhaltigere Welt zu schaffen und mit nachhaltigkeitsrelevanten Problemstellungen umzugehen, wie sie in den SDGs beschrieben werden, müssen Individuen Change Agents für eine nachhaltige Entwicklung werden. Sie benötigen das Wissen, die Fähigkeiten, die Werte und die Einstellungen, die sie dazu in die Lage versetzen, zu einer nachhaltigen Entwicklung beizutragen. Bildung ist daher entscheidend für das Erreichen einer nachhaltigen Entwicklung. Doch nicht jede Art von Bildung unterstützt eine nachhaltige Entwicklung. Bildung, die das Wirtschaftswachstum allein fördert, kann auch zu einer Zunahme nicht nachhaltiger Konsummuster führen. Das mittlerweile gut etablierte Konzept einer Bildung für nachhaltige Entwicklung (BNE) ermächtigt die Lernenden, fundierte Entscheidungen für den Erhalt der Umwelt, wirtschaftliche Leistungsfähigkeit und eine gerechte Gesellschaft für gegenwärtige und zukünftige Generationen zu treffen und entsprechend verantwortungsbewusst zu handeln (Rieckmann, 2016). BNE zielt darauf ab, Kompetenzen zu entwickeln, die es den Individuen ermöglichen, über ihre eigenen Handlungen unter Berücksichtigung ihrer gegenwärtigen und zukünftigen sozialen, kulturellen, wirtschaftlichen und ökologischen Auswirkungen - unter lokaler und globaler Perspektive - nachzudenken, in komplexen Situationen nachhaltig zu handeln, was von dem Individuum erfordern kann, neue Richtungen einzuschlagen und sich an gesellschaftspolitischen Prozessen zu beteiligen, die ihre Gesellschaften in Richtung einer nachhaltigen Entwicklung bewegen (de Haan, 2010; de Haan et al., 2008; Overwien, 2013; Rieckmann, 2013, 2016, 2018).

BNE muss als integraler Bestandteil hochwertiger Bildung verstanden werden, die ein Element des Konzepts lebenslangen Lernens ist: Alle Bildungseinrichtungen - vom Vorschul- bis zum Hochschulbereich bis hin zur non-formalen und informellen Bildung - können und sollten es als ihre Verantwortung betrachten, sich intensiv mit Fragen einer nachhaltigen Entwicklung zu beschäftigen und die Entwicklung von Nachhaltigkeitskompetenzen zu fördern (Nationale Plattform Bildung für nachhaltige Entwicklung, 2017; Rieckmann, 2016). BNE ermöglicht Bildungs- und Lernprozesse, die für jeden Lernenden angesichts der heutigen Herausforderungen sehr relevant sind. 
Die internationale Anerkennung von BNE als wichtiges Instrument für die Förderung einer nachhaltigen Entwicklung ist stetig gewachsen. Diese Bedeutung von BNE wurde auch auf den drei wichtigsten globalen Gipfeltreffen für nachhaltige Entwicklung betont: die UN-Konferenz über Umwelt und Entwicklung (UNCED) 1992 in Rio de Janeiro, der Weltgipfel für Nachhaltige Entwicklung (WSSD) 2002 in Johannesburg und die UN-Konferenz für Nachhaltige Entwicklung (UNCSD) 2012 ebenfalls in Rio de Janeiro. Die UN-Dekade Bildung für nachhaltige Entwicklung (2005-2014) zielte darauf ab, die Prinzipien und Praktiken einer nachhaltigen Entwicklung umfassend in Bildungs- und Lernprozesse zu integrieren, um Veränderungen von Wissen, Werten und Einstellungen im Sinne der Vision einer nachhaltigeren und gerechteren Gesellschaft für alle zu fördern. Das Weltaktionsprogramm (WAP) Bildung für nachhaltige Entwicklung, das am 12. November 2014 auf der UNESCO-Weltkonferenz zu BNE (Aichi-Nagoya, Japan) lanciert wurde, strebt danach, auf der Grundlage der Ergebnisse der Dekade, die Umsetzung von BNE zu intensivieren (UNESCO, 2014).

BNE wird in den SDGs ausdrücklich als Teil des Ziels 4.7 des SDG zu hochwertiger Bildung anerkannt (Abb. 2) - zusammen mit Global Citizenship Education (GCED), die die UNESCO als einen komplementären Ansatz fördert (UNESCO, 2015a).

\section{Abb. 2: Ziel 4.7 der SDGs}

Bis 2030 sicherstellen, dass alle Lernenden die notwendigen Kenntnisse und Qualifikationen zur Förderung nachhaltiger Entwicklung erwerben, unter anderem durch Bildung für nachhaltige Entwicklung und nachhaltige Lebensweisen, Menschenrechte, Geschlechtergleichstellung, eine Kultur des Friedens und der Gewaltlosigkeit, Weltbürgerschaft und die Wertschätzung kultureller Vielfalt und des Beitrags der Kultur zu nachhaltiger Entwicklung.

Quelle: Vereinte Nationen, 2015, S. 19

Gleichzeitig ist es wichtig, die zentrale Bedeutung von BNE für alle anderen 16 SDGs zu betonen. Mit ihrem Ziel, übergreifende Nachhaltigkeitskompetenzen bei den Lernenden zu entwickeln, leistet BNE einen wesentlichen Beitrag für alle Bemühungen zum Erreichen der SDGs. Denn BNE befähigt Individuen, an einer nachhaltigen Entwicklung mitzuwirken, indem sie den gesellschaftlichen, wirtschaftlichen und politischen Wandel fördern und ihr eigenes Verhalten ändern. BNE kann spezifische kognitive, sozioemotionale und verhaltensbezogene Lernergebnisse erzielen, die es Individuen ermöglichen, mit den besonderen Herausforderungen jedes SDG umzugehen und so dessen Umsetzung zu unterstützen. BNE ermöglicht es allen Individuen, zur Erreichung der SDGs beizutragen, indem sie mit den notwendigen Kenntnissen und Kompetenzen ausgestattet werden, die sie nicht nur brauchen, um zu verstehen, worum es bei den SDGs geht, sondern auch um sich als informierte Bürgerinnen und Bürger für die Herbeiführung der notwendigen Transformation einzusetzen (UNESCO, 2017).

\section{Lernziele für das Erreichen des SDGs}

BNE kann übergreifende Nachhaltigkeitskompetenzen entwickeln, die für alle SDGs relevant sind. Zudem kann BNE auch spezifische Lernergebnisse erzielen, die wir benötigen, um zum Erreichen eines bestimmten SDGs beizutragen.

Schlüsselkompetenzen für das Erreichen der SDGs Die Weltgesellschaft steht vor vielen Herausforderungen: eine zunehmende Komplexität und Unsicherheit, mehr Individualisierung und soziale Vielfalt, wachsende wirtschaftliche und kulturelle Einheitlichkeit, eine Verschlechterung der Ökosystemdienstleistungen sowie eine zunehmende Anfälligkeit und Exposition gegenüber natürlichen und technologischen Gefahren. Vor diesem Hintergrund müssen die Menschen lernen, die komplexe Welt zu verstehen, in der sie leben. Sie müssen in der Lage sein, zusammenzuarbeiten, ihre Meinung zu äußern und sich für positive Veränderungen einzusetzen (UNESCO, 2015b). Es geht darum, „Nachhaltigkeitsbürger*innen“ (Rieckmann \& Schank, 2016; Wals, 2015; Wals \& Lenglet, 2016) zu werden. „Nachhaltigkeitsbürger*innen“ benötigen bestimmte Schlüsselkompe-

\section{Abb. 3: Nachhaltigkeitskompetenzen}

- Kompetenz zum vernetzten Denken: die Fähigkeiten, Zusammenhänge zu erkennen und zu verstehen; komplexe Systeme zu analysieren; zu überlegen, wie Systeme in verschiedene Domänen und verschiedene Skalen eingebettet sind; und mit Unsicherheit umzugehen Kompetenz zum vorausschauenden Denken: die Fähigkeiten, multiple (mögliche, wahrscheinliche und wünschenswerte) Zukünfte zu verstehen und zu bewerten; eigene Visionen für die Zukunft zu schaffen; das Vorsorgeprinzip anzuwenden; die Konsequenzen von Handlungen zu beurteilen; und mit Risiken und Veränderungen umzugehen Normative Kompetenz: die Fähigkeiten, die Normen und Werte zu verstehen und zu reflektieren, die den eigenen Handlungen zugrunde liegen; und Nachhaltigkeitswerte, Prinzipien und Ziele im Kontext von Interessenkonflikten und Trade-offs, unsicheren Kenntnissen und Widersprüchen zu verhandeln

Strategische Kompetenz: die Fähigkeiten zur kollektiven Entwicklung und Umsetzung innovativer Maßnahmen, die Nachhaltigkeit auf lokaler Ebene und darüber hinaus voranbringen

- Kooperationskompetenz: die Fähigkeiten, von anderen zu lernen; die Bedürfnisse, Perspektiven und Handlungen anderer zu verstehen und zu respektieren (Empathie), eine Beziehung zu ihnen aufzubauen und für sie empfindsam zu sein (empathische Führung); mit Konflikten in einer Gruppe umzugehen; und eine kollaborative und partizipative Problemlösung zu ermöglichen

- Kompetenz zum kritischen Denken: die Fähigkeit, Normen, Praktiken und Meinungen zu hinterfragen; die eigenen Werte, Wahrnehmungen und Handlungen zu reflektieren; und sich im Nachhaltigkeitsdiskurs zu positionieren

Selbstkompetenz: die Fähigkeit, über die eigene Rolle in der lokalen Gemeinschaft und (globalen) Gesellschaft nachzudenken; kontinuierlich seine Handlungen zu bewerten und sich weiter zu motivieren; und sich mit den eigenen Gefühlen und Wünschen auseinanderzusetzen

Integrierte Problemlösekompetenz: die übergreifende Fähigkeit, unterschiedliche Problemlösungsrahmen für komplexe Nachhaltigkeitsprobleme anzuwenden und passfähige, inklusive und gerechte Lösungsmöglichkeiten zu entwickeln, die eine nachhaltige Entwicklung fördern und die oben genannten Kompetenzen integrieren

Quelle: UNESCO, 2017 
tenzen (Rychen, 2003; Weinert, 2001), die es ihnen ermöglichen, konstruktiv und verantwortungsvoll mit der heutigen Welt umzugehen. Einige Schlüsselkompetenzen werden im internationalen BNE-Diskurs als besonders wichtig für eine nachhaltige Entwicklung angesehen (Abb. 3) (de Haan, 2010; de Haan et al., 2008; Rieckmann, 2012, 2013, 2018; Wiek et al., 2011, 2016).

Die Nachhaltigkeitskompetenzen machen deutlich, was Nachhaltigkeitsbürger/-innen insbesondere benötigen, um sich mit den heutigen komplexen Herausforderungen auseinanderzusetzen. Sie sind für alle SDGs relevant und ermöglichen es den Individuen, die verschiedenen SDGs in Beziehung zueinander zu setzen, um das große Ganze der Agenda 2030 für Nachhaltige Entwicklung zu erkennen (UNESCO, 2017).

\section{Spezifische Lernziele für die SDGs}

Während die Nachhaltigkeitskompetenzen dazu befähigen, insgesamt mit den SDGs umzugehen, lässt sich für jedes einzelne SDG die Frage stellen, wie damit verbundene, spezifische Lernprozesse gestaltet werden können.

Dazu bietet der von der UNESCO (2017) veröffentliche Leitfaden „Education for Sustainable Development Goals. Learning Objectives“ vielfältige Anregungen. Dieses Dokument zielt darauf ab, politische Entscheidungsträger/innen, Curriculum-Entwickler/innen und Lehrende bei der Gestaltung von Strategien, Lehrplänen und Bildungsveranstaltungen zu unterstützen, um Lernen für die SDGs zu fördern. Im Sinne einer allgemeinen Orientierung sind die Lernziele, Themen und Aktivitäten für jedes SDG, nicht für spezifische Altersgruppen, Lernumgebungen oder bestimmte soziokulturelle Kontexte, zugeschnitten. Sie sind so konzipiert, dass sie für alle Lernenden aller Altersgruppen weltweit relevant sind und ihre Anwendung in allen möglichen Lernumgebungen finden können. Es handelt sich also um Vorschläge für Lernziele und -inhalte, die Pädagoginnen und Pädagogen auswählen und an ihre konkreten Lehr-Lern-Kontexte (soziokulturelle Anforderungen, Zielgruppen, etc.) anpassen können. Es werden für jedes SDG Lernziele in einer kognitiven, sozioemotionalen und verhaltensbezogenen Domäne beschrieben (UNESCO, 2017):

- Die kognitive Domäne umfasst Wissen und kognitive Fähigkeiten, die notwendig sind, um das SDG und die mit seinem Erreichen verbundenen Herausforderungen besser zu verstehen.

- Die sozioemotionale Domäne umfasst soziale Kompetenzen, die es Lernenden ermöglichen, zusammenzuarbeiten, zu verhandeln und zu kommunizieren, um das SDG voranzubringen, sowie Selbstreflexionsfähigkeiten, Werte, Einstellungen und Motivationen, die es den Lernenden ermöglichen, sich selbst weiterzuentwickeln.

- Die verhaltensbezogene Domäne beschreibt Handlungskompetenzen.

- Darüber hinaus werden für jedes SDG beispielhafte Themen und pädagogische Ansätze skizziert.

Zur Verdeutlichung dieses Ansatzes sollen hier exemplarisch die Lernziele zum SDG 1 „Keine Armut - Armut in jeder Form und überall beenden" dargestellt werden (Abb. 4).

Abb. 4: Lernziele für das SDG 1 „Keine Armut“

\begin{tabular}{|c|c|}
\hline $\begin{array}{l}\text { Cognitive learning } \\
\text { objectives }\end{array}$ & $\begin{array}{l}\text { 1. The learner understands the concepts of extreme and relative poverty and is able to critically reflect on their underlying cultural and } \\
\text { normative assumptions and practices. } \\
\text { 2. The learner knows about the local, national and global distribution of extreme poverty and extreme wealth. } \\
\text { 3. The learner knows about causes and impacts of poverty such as unequal distribution of resources and power, colonization, conflicts, } \\
\text { disasters caused by natural hazards and other climate change-induced impacts, environmental degradation and technological disa- } \\
\text { sters, and the lack of social protection systems and measures. } \\
\text { 4. The learner understands how extremes of poverty and extremes of wealth affect basic human rights and needs. } \\
\text { 5. The learner knows about poverty reduction strategies and measures and is able to distinguish between deficit-based and strength-ba- } \\
\text { sed approaches to addressing poverty. }\end{array}$ \\
\hline $\begin{array}{l}\text { Socio-emotional } \\
\text { learning objectives }\end{array}$ & $\begin{array}{l}\text { 1. The learner is able to collaborate with others to empower individuals and communities to affect change in the distribution of power } \\
\text { and resources in the community and beyond. } \\
\text { 2. The learner is able to raise awareness about extremes of poverty and wealth and encourage dialogue about solutions. } \\
\text { 3. The learner is able to show sensitivity to the issues of poverty as well as empathy and solidarity with poor people and those in vulne- } \\
\text { rable situations. } \\
\text { 4. The learner is able to identify their personal experiences and biases with respect to poverty. } \\
\text { 5. The learner is able to critically reflect on their own role in maintaining global structures of inequality. }\end{array}$ \\
\hline $\begin{array}{l}\text { Behavioural learning } \\
\text { objectives }\end{array}$ & $\begin{array}{l}\text { 1. The learner is able to plan, implement, evaluate and replicate activities that contribute to poverty reduction. } \\
\text { 2. The learner is able to publicly demand and support the development and integration of policies that promote social and economic } \\
\text { justice, risk reduction strategies as well as poverty eradication actions. } \\
\text { 3. The learner is able to evaluate, participate in and influence decision-making related to management strategies of local, national and } \\
\text { international enterprises concerning poverty generation and eradication. } \\
\text { 4. The learner is able to include poverty reduction, social justice and anti-corruption considerations in their consumption activities. } \\
\text { 5. The learner is able to propose solutions to address systemic problems related to poverty. }\end{array}$ \\
\hline
\end{tabular}


Um dieses Wissen und die genannten Fähigkeiten bei den Lernenden zu entwickeln, bieten sich einige Inhalte besonders an (Abb. 5). Dabei handelt es sich um keine erschöpfende oder endgültige Auflistung von Themen, sondern um Vorschläge von weltweit relevanten Aspekten des jeweiligen SDG. Diese sollten durch jeweils lokal relevante Themen ergänzt und mit neuen Themen aktualisiert werden, die sich ständig in unserer sich schnell verändernden Welt ergeben.

Abb. 5: Vorschläge für Themen zur Bearbeitung des SDG 1 „Keine Armut“

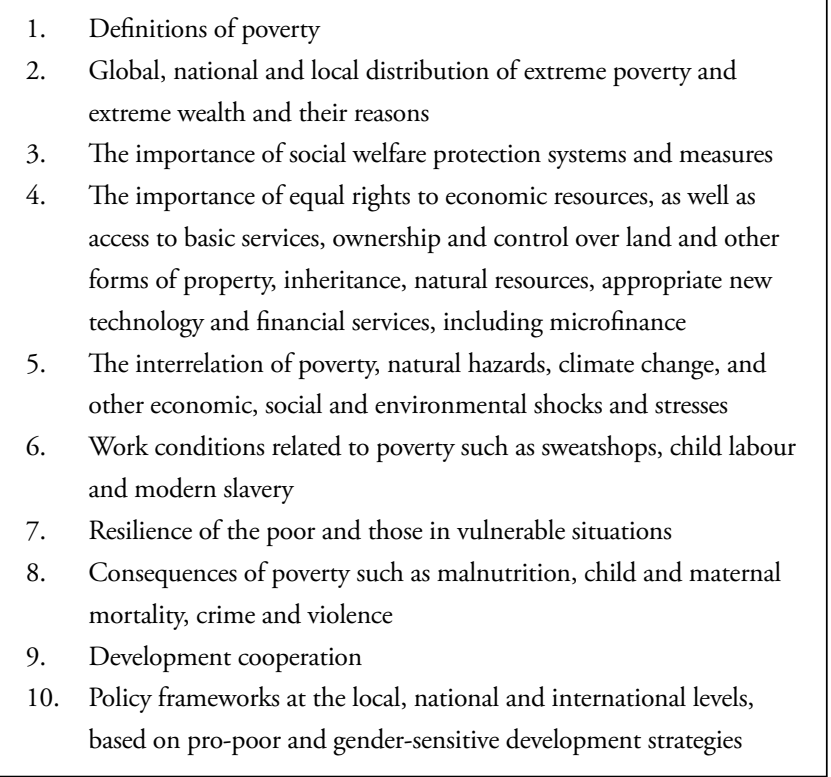

Quelle: UNESCO, 2017, S. 13

Ebenso werden einige Methoden vorgestellt, die - neben vielen anderen möglichen Methoden - zur Bearbeitung der Themen und der Entwicklung der jeweiligen Lernziele geeignet sind (Abb. 6).

Die für jedes SDG beschriebenen, spezifischen Lernziele sind immer in Verbindung mit den übergreifenden Nachhaltigkeitskompetenzen zu sehen. Denn erst die Schlüsselkompetenzen ermöglichen es den Lernenden, die komplexen Zusammenhänge und auch Widersprüche zwischen den 17 SDGs zu erkennen und zu verstehen.

\section{Handlungsorientierte transformative Pädagogik}

Für die Entwicklung der Nachhaltigkeitskompetenzen und das Erreichen der Lernziele für die einzelnen SDGs bedarf es einer handlungsorientierten, transformativen Pädagogik, die sich durch Aspekte wie selbstgesteuertes Lernen, Partizipation und Kooperation, Problemorientierung, Inter- und Transdisziplinarität und die Verknüpfung von formalem und informellem Lernen auszeichnet (UNESCO, 2017). Nur solche pädagogischen Ansätze ermöglichen den Erwerb derjenigen Schlüsselkompetenzen, die für die Förderung einer nachhaltigen Entwicklung erforderlich sind (Rieckmann, 2016, 2018). Einer Bildung für nachhaltige Entwicklung geht es darum, die Lernenden zu befähigen und zu motivieren, aktive Nachhaltigkeitsbürger/innen zu werden, die in der Lage sind, kritisch zu denken und sich an der Gestaltung einer nachhaltigen Zukunft zu beteiligen. Pädagogische
Abb. 6: Beispielhafte Lernansätze und-methoden für das SDG 1 „Keine Armut"

1. Develop partnerships between schools and universities in different regions of the world (South and North; South and South).

2. Plan and run an awareness campaign about poverty locally and globally.

3. Plan and run a student company selling fair trade products.

4. Plan and implement local service-learning and/or engagement opportunities for empowering poor people, reducing their vulnerability to different hazards and increasing their resilience - in collaboration with NGOs, the private sector and/or community groups, etc.

5. Conduct a case study on poverty and wealth in selected countries (through desktop research) or at the local level (through excursions, doing interviews, etc.).

6. Provide internships within organisations addressing poverty.

7. Develop an enquiry-based project around: "Is poverty increasing or decreasing?"

Quelle: UNESCO, 2017, S. 13

Ansätze, die diesem Ziel entsprechen, sind lernerzentriert, handlungsorientiert und transformativ (Abb. 7). Diese pädagogischen Ansätze stellen allgemeine Prinzipien für die Gestaltung von Lernprozessen in der BNE dar. Spezifische Methoden, die diesen Prinzipien entsprechen, sind erforderlich, um den Lernprozess zu ermöglichen.

In der BNE werden Methoden bevorzugt, die die Entwicklung von Kompetenzen durch aktives Lernen fördern. Bestimmte Methoden können für BNE besonders empfohlen werden (Abb. 8). Diese partizipativen Lehr- und Lernmethoden befähigen die Lernenden, sich an der Gestaltung einer nachhaltigen Entwicklung zu beteiligen. Um vielfältige und grenzüberschreitende Lernumgebungen zu schaffen und umfassende Bilder der SDGs zu entwickeln, sollten Bildungseinrichtungen und Lehrende Partnerschaften auf lokaler, nationaler und internationaler Ebene fördern (UNESCO, 2017). Es ist wichtig zu erkennen, dass angemessene Reaktionen auf Nachhaltigkeitsprobleme nicht auf einzelne Perspektiven, Disziplinen oder Arten des Wissens beschränkt werden können. Partnerschaften, die eine Reihe von gesellschaftlichen Akteuren wie Unternehmen, NGOs, öffentliche Institutionen, politische Entscheidungsträger und/oder Einzelpersonen einbeziehen, eröffnen neue Lernmöglichkeiten. In einem Dialog oder einem Projekt, das die Zusammenarbeit mit einem Praxispartner beinhaltet, können die Lernenden etwas über realweltliche Herausforderungen erfahren und von den Kompetenzen und Erfahrungen der Partner profitieren (Barth, Adomßent, Fischer, Richter \& Rieckmann, 2014). Partnerschaften zwischen Lernenden aus der ganzen Welt fördern den Austausch unterschiedlicher Perspektiven und Kenntnisse zu demselben Thema. Zum Beispiel können virtuelle Lehrveranstaltungen eine Umgebung schaffen, um einen globalen Dialog zu üben und gegenseitigen Respekt und Verständnis zu fördern (Barth \& Rieckmann, 2009).

\section{Fazit}

Für das Erreichen der SDGs kommt BNE eine wichtige Bedeutung zu. Sie kann die Entwicklung von Nachhaltigkeitskompe- 
Abb. 7: Zentrale pädagogische Ansätze einer BNE

\begin{abstract}
Lernerzentrierte Ansätze
Die lernerzentrierte Pädagogik geht von selbstständigen Lernenden aus und betont die aktive Entwicklung des Wissens anstelle eines bloßen Wissenstransfers und/oder passiven Lernerfahrungen. Das Vorwissen der Lernenden sowie ihre Erfahrungen im sozialen Kontext sind Ausgangspunkte für die Förderung von Lernprozessen, in denen die Lernenden ihre eigene Wissensbasis konstruieren. Lernerzentrierte Ansätze erfordern von den Lernenden, über ihre eigenen Kenntnisse und Lernprozesse nachzudenken, um sie zu steuern und zu verfolgen. Lehrende sollten diese Reflexionen anregen und unterstützen. Lernerzentrierte Ansätze verändern die Rolle der/des Lehrenden hin zu Moderator/inn/en von Lernprozessen (Barth, 2015).

Handlungsorientiertes Lernen

Im handlungsorientierten Lernen werden die Lernenden selbst tätig und reflektieren ihre Erfahrungen in Bezug auf den beabsichtigten Lernprozess und die angestrebte persönliche Entwicklung. Diese Erfahrungen könnten sich aus einem Projekt, einem Praktikum, der Durchführung eines Workshops, einer Kampagne etc. ergeben. Handlungsorientiertes Lernen bezieht sich auf Kolbs Theorie des erfahrungsbasierten Lernzyklus (vgl. weiterführend Kolb, 1984) und steigert den Wissenserwerb, die Kompetenzentwicklung und die Klärung von Werten durch die Verknüpfung von abstrakten Konzepten mit persönlicher Erfahrung und dem Leben des Lernenden. Die Rolle des/der Lehrenden besteht darin, eine Lernumgebung zu schaffen, die die Erfahrungen der Lernenden und reflexive Denkprozesse anregt.

Transformatives Lernen

Das transformative Lernen zielt darauf $a b$, die Lernenden dazu zu befähigen, die Art und Weise zu hinterfragen und zu verändern, wie sie die Welt sehen und über sie denken, um ihr Verständnis von ihr zu erweitern (Slavich \& Zimbardo, 2012; Mezirow, 2000). Der/die Lehrende ist ein Facilitator, der die Lernenden befähigen soll, ihre Weltanschauungen zu verändern. Das verwandte Konzept des transgressiven Lernens (Lotz-Sisitka, Wals, Kronlid \& McGarry, 2015) geht noch einen Schritt weiter: Es betont, dass das Lernen in der BNE den Status quo überwinden und die Lernenden für disruptives (störendes) Denken und die Co-Kreation neuen Wissens vorbereiten sollte.
\end{abstract}

Quelle: UNESCO, 2017, S. 12

tenzen fördern, die Individuen nicht nur dazu befähigen, die SDGs in ihrer ganzen Komplexität zu verstehen, sondern auch eigene Beiträge zum Erreichen der Ziele zu leisten. Gleichzeitig kann BNE auch spezifische Lernprozesse ermöglichen, die dazu benötigt werden, sich ein bestimmtes SDG zu erschließen und eigene Beiträge zu diesem Ziel zu leisten. Zudem bieten die SDGs eine inhaltliche Orientierung für BNE. Sie zeigen wesentliche Themenstellungen auf, die es im Rahmen einer BNE zu bearbeiten gilt. Für die Entwicklung der Nachhaltigkeitskompetenzen und eine Auseinandersetzung mit den einzelnen SDGs bedarf es einer handlungsorientierten, transformativen Pädagogik. Jedoch sollte BNE auch Räume für eine kritische Reflexion über die SDGs eröffnen. So kann es z.B. darum gehen, mit Lernenden die den SDGs inhärenten Widersprüche (u.a. in Bezug auf Wirtschaftswachstum, Messner \& Scholz, 2015) bzw. Leerstellen (u.a. „Dekarbonisierung“, „planetare Grenzen“, „Benennung und Bearbeitung von Ursachen hinter den zu lösenden
Problemlagen“, Messner \& Scholz, 2015, S. 23; Scholz, 2017) zu analysieren und zu diskutieren. Eine nachhaltige Entwicklung selbst ist ein Lernprozess, und Bildung für nachhaltige Entwicklung - verstanden als emanzipatorisches Bildungskonzept sollte dazu beitragen, die Ziele und Grundlagen einer nachhaltigen Entwicklung immer wieder zu hinterfragen und weiterzuentwickeln.

Abb. 8: Schlüsselmethoden für das Lernen der SDGs

- Kollaborative Projekte in Ernstsituationen wie Service-LearningProjekte und Kampagnen für verschiedene SDGs

- Methoden zur Entwicklung von Zukunftsentwürfen wie Zukunftswerkstätten, Szenarioanalysen, utopische/dystopische Erzählungen, Science-Fiction-Denken sowie Prognosen und Backcasting

- Analysen komplexer Systeme durch partizipative Forschungsprojekte, Fallstudien, Stakeholder-Analysen, Akteursanalysen, Modellierung, Systemspiele, etc.

- Kritisches und reflektierendes Denken durch Fisch-Bowl-Diskussionen, Lerntagebücher, etc.

Quelle: UNESCO, 2017

Um mit BNE zur Förderung der SDGs beizutragen und sie aber auch kritisch zu reflektieren, ist es allerdings notwendig, dass BNE als Querschnittsanliegen in alle Bildungsbereiche integriert wird. Dieser Anspruch wird im Nationalen Aktionsplan Bildung für nachhaltige Entwicklung (Nationale Plattform Bildung für nachhaltige Entwicklung, 2017) formuliert und es sind in den letzten Jahren Entwicklungen in diese Richtung zu verzeichnen gewesen.

Weiterhin stellt es aber eine große Herausforderung dar, BNE als orientierendes Bildungskonzept in den Strukturen und der Praxis des Bildungssystems zu verankern.

\section{Literatur}

Barth, M. (2015). Implementing sustainability in higher education: learning in an age of transformation. London: Routledge.

Barth, M., Adomßent, M., Fischer, D., Richter, S. \& Rieckmann, M. (2014) Learning to change universities from within: a service-learning perspective on promoting sustainable consumption in higher education. Journal of Cleaner Production, 62, 72-81.

Barth, M. \& Rieckmann, M. (2009). Experiencing the Global Dimension of Sustainability: Student Dialogue in a European-Latin American Virtual Seminar. International Journal of Development Education and Global Learning, 1(3), 23-38.

de Haan, G. (2010). The development of ESD-related competencies in supportive institutional frameworks. International Review of Education, 56(2), 315-328.

de Haan, G., Kamp, G., Lerch, A., Martignon, L., Müller-Christ, G. \& Nutzinger, H.-G. (Hrsg.) (2008). Nachhaltigkeit und Gerechtigkeit. Grundlagen und schulpraktische Konsequenzen. Berlin \& Heidelberg: Springer.

Kercher, J. (2015). Wie die Nachhaltigkeitsziele in die Welt kamen. Von den MDGs zu den SDGs. In oekom e.V. - Verein für ökologische Kommunikation (Hrsg.), Nachhaltige Entwicklungsziele. Agenda für eine bessere Welt? (S. 27-33). München: Oekom.

Kolb, D. A. (1984). Experiential Learning: Experience as the Source of Learning and Development. Englewood Cliffs, N.J.: Prentice-Hall.

Lotz-Sisitka, H., Wals, A. E., Kronlid, D. \& McGarry, D. (2015). Transformative, transgressive social learning: rethinking higher education pedagogy in times of systemic global dysfunction. Current Opinion in Environmental Sustainability, 16, 73-80.

Messner, D. \& Scholz, I. (2015). Gemeinsam für das Wohlergehen aller. Agenda 2030 und die Sustainable Development Goals. In oekom e.V. - Verein für ökologische Kommunikation (Hrsg.), Nachhaltige Entwicklungsziele. Agenda für eine bessere Welt? (S. 18-26)München: Oekom. 
Mezirow, J. (2000). Learning as transformation: critical perspectives on a theory in progress. San Francisco: Jossey-Bass.

Nationale Plattform Bildung für nachhaltige Entwicklung (2017). Nationaler Aktionsplan Bildung für nachhaltige Entwicklung. Der deutsche Beitrag zum UNESCOWeltaktionsprogramm. Zugriff am 18.05.2018 http://www.bne-portal.de/sites/ default/files/Nationaler_Aktionsplan_BNE_2017.pdf

Overwien, B. (2013). Kompetenzmodelle im Lernbereich „Globale Entwicklung“ - Bildung für nachhaltige Entwicklung. In B. Overwien \& H. Rode (Hrsg.), Bildung für nachhaltige Entwicklung. Lebenslanges Lernen, Kompetenz und gesellschaftliche Teilhabe (S. 13-34). Leverkusen: Budrich.

Rieckmann, M. (2012). Future-oriented higher education: Which key competencies should be fostered through university teaching and learning? Futures, 44(2), $127-135$.

Rieckmann, M. (2013). Schlüsselkompetenzen für eine nachhaltige Entwicklung. POLIS, 2013(4), 11-14.

Rieckmann, M. (2018). Chapter 2 - Learning to transform the world: key competencies in ESD. In A. Leicht, J. Heiss \& W. J. Byun (Hrsg.), Issues and trends in Education for Sustainable Development (S. 39-59). Paris: UNESCO.

Rieckmann, M. \& Schank, C. (2016). Sozioökonomisch fundierte Bildung für nachhaltige Entwicklung - Kompetenzentwicklung und Werteorientierungen zwischen individueller Verantwortung und struktureller Transformation. SOCIENCE, 1(1), 65-79.

Rychen, D. S. (2003). Key competencies: Meeting important challenges in life. In D. S. Rychen \& L. H. Salganik (Hrsg.), Key competencies for a successful life and well-functioning society (S. 63-107). Cambridge/ MA: Hogrefe and Huber.

Scholz, I. (2015). Universelle Ziele für eine nachhaltige Entwicklung. In: H. Leitschuh, G. Michelsen, U. E. Simonis, J. Sommer \& E. U. von Weizsäcker (Hrsg.), Gesucht: Weltumweltpolitik. Herausforderungen im Anthropozän (S. 116-125). Stuttgart: Hirzel.

Scholz, I. (2017). Herausforderung Sustainable Development Goals. In G. Michelsen (Hrsg.), Die deutsche Nachhaltigkeitsstrategie. Wegweiser für eine Politik der Nachhaltigkeit (S. 23-39). Wiesbaden: Hessische Landeszentrale für politische Bildung Slavich, G. M. \& Zimbardo, P. G. (2012). Transformational Teaching: Theoretical Underpinnings. Basic Principles, and Core Methods. Educational Psychology Review, 24(4), 569-608.

UNESCO (2014): UNESCO Roadmap zur Umsetzung des Weltaktionsprogramms „Bildung für nachhaltige Entwicklung". Zugriff am 18.05.2018 https://www.bmbf. de/files/2015_Roadmap_deutsch.pdf

UNESCO (2015a): Global Citizenship Education: Topics and learning objectives. Zugriff am 18.05.2018 http://unesdoc.unesco.org/images/0023/002329/232993e.pdf
UNESCO (2015b): Rethinking Education. Towards a global common good? Zugriff am 18.05.2018 http://unesdoc.unesco.org/images/0023/002325/232555e.pdf

UNESCO (2017). Education for Sustainable Development Goals. Learning Objectives. Zugriff am 18.05.2018 http://unesdoc.unesco.org/images/0024/002474/ $247444 \mathrm{e}$ pdf

Vereinte Nationen (2015). Transformation unserer Welt: die Agenda 2030 für nachhaltige Entwicklung. Zugriff am 18.05.2018 http://www.un.org/depts/german/gv70/band1/ar70001.pdf

Wals, A. E. J. (2015). Beyond unreasonable doubt. Education and learning for socio-ecological sustainability in the Anthropocene. Zugriff am 18.05.2018https://arjenwals. files.wordpress.com/2016/02/8412100972_rvb_inauguratie-wals_oratieboekje_ v02.pdf, Abruf: 18.05.2018

Wals, A. E. J. \& Lenglet, F. (2016). Sustainability citizens: Collaborative and disruptive social learning. In R. Horne, J. Fien, B. B. Beza \& A. Nelson (Hrsg.), Sustainability Citizenship in Cities: Theory and Practice (S. 52-67). London: Routledge.

Weinert, F. E. (2001). Concept of Competence: A Conceptual Clarification. In D. S. Rychen \& L. H. Salganik (Hrsg.), Defining and Selecting Key Competencies (S. 45-65). Seattle, Toronto, Bern \& Göttingen: Hogrefe und Huber.

Wiek, A., Withycombe, L. \& Redman, C. L. (2011): Key competencies in sustainability: a reference framework for academic program development. Sustainability Science, 6(2), 203-218.

Wiek, A., Bernstein, M. J., Foley, R. W., Cohen, M., Forrest, N., Kuzdas, C., Kay, B. \& Withycombe Keeler, L. (2016): Operationalising competencies in higher education for sustainable development. In M. Barth, G. Michelsen, I. Thomas \& M. Rieckmann (Hrsg.), Routledge Handbook of Higher Education for Sustainable Development (S. 241-260). London: Routledge.

\section{Prof. Dr. Marco Rieckmann}

ist Professor für Hochschuldidaktik, Schwerpunkt Schlüsselkompetenzen, im Fach Erziehungswissenschaften der Fakultät I - Bildungs- und Gesellschaftswissenschaften an der Universität Vechta. Zuvor war er wissenschaftlicher Mitarbeiter an der Leuphana Universität Lüneburg (2004-2013). Er ist Vertreter der Deutschen Gesellschaft für Erziehungswissenschaft (DGfE) im Council der European Educational Research Association (EERA) sowie Sprecher des Deutschsprachigen Netzwerks „LehrerInnenbildung für nachhaltige Entwicklung" (LeNa). Seine Arbeitsschwerpunkte sind: Hochschuldidaktik, (Hochschul-)Bildung für nachhaltige Entwicklung, Nachhaltige Hochschulentwicklung. 


\section{Die Bildungsagenda der Vereinten Nationen aus dem Blickwinkel der internationalen Bildungsforschung}

\begin{abstract}
Zusammenfassung
Die von den Vereinten Nationen 2015 ausgerufenen Sustainable Development Goals (SDGs), d.h. die Entwicklungsziele für eine nachhaltige weltweite Entwicklung, die von 2016 bis zum Jahre 2030 umgesetzt werden sollen, enthalten ein eigens dem Bereich Erziehung und Bildung gewidmetes Ziel Vier (SDG 4), das da lautet: „Bis 2030 für alle Menschen inklusive, chancengerechte und hochwertige Bildung sicherstellen sowie Möglichkeiten zum lebenslangen Lernen fördern." Im folgenden Beitrag wird das damit aufgeworfene Thema aus der Perspektive der internationalen Bildungsforschung in den Blick genommen. Dies geschieht zunächst durch eine historische Kontextualisierung mit anderen UN-Programmen seit den 1990er Jahren, um dadurch die Bedeutsamkeit des SDG 4 besser einordnen zu können. Sodann sollen die insgesamt zehn Unterpunkte des SDG 4 nach möglichst präzisen Aussagen zu den angestrebten Entwicklungen durchforstet werden, um sich jenseits der genretypisch eher allgemeinen und vagen Formulierungen ein Bild davon machen zu können, was unter den Zielvorstellungen verstanden wird und tatsächlich intendiert ist. Danach geht es um die inzwischen im System der Vereinten Nationen begonnene Arbeit an den Indikatoren, mit denen die Realisierung des SDG 4 weltweit zwecks jährlicher Berichterstattung erhoben und verglichen werden soll. Abschließend wird die potentielle Anschlussfähigkeit des SDG 4-Monitoring an Forschung und Lehre im Rahmen einer internationalen Bildungsforschung reflektiert.
\end{abstract}

Schlüsselworte: SDG 4, Bildungsagenda 2030, UNESCO, Nachhaltige Entwicklung, Bildung für Alle

\footnotetext{
Abstract

The Sustainable Development Goals (SDGs) declared in 2015 by the United Nations to be globally implemented by 2030 encompass one goal which is specifically devoted to a broad range of education and instruction - goal four (SDG 4) which reads: "Ensure inclusive and equitable quality education and promote lifelong learning opportunities for all". The following article focuses this topic from the perspective of international
}

research in education. In a first point this is realised by placing the SDG 4 into its broader historical context with other UNprograms since the 1990s. Programmatic declarations such as the SDGs are often employing rather vague language. In a next step it will therefore be clarified how the SDG 4 is turned to be operative into its ten subdivided features by screening this document for its most precise visions for education. Following this, attention will be called to the UN-led establishment and implementation of sets of indicators which are essential for the monitoring and reporting of the SDG Agendas including SDG 4. The article will conclude with remarks on how the SDG 4 and its context might be relevant for research and lecturing on international dimensions of education.

Keywords: SDG 4, Education 2030 Agenda, UNESCO, Sustainable Development, Education for All

\section{Das SDG 4: seine Prinzipien und seine Vorgeschichte}

Die auf das Jahr 2030 ausgerichtete Nachhaltigkeitsagenda der Vereinten Nationen umfasst 17 Ziele (Sustainable Development Goals, SDGs), darunter eines für Bildung: das SDG 4. Alternativ zur Rede über „das SDG 4“ hat sich in der internationalen wie in der deutschsprachigen Diskussion inzwischen auch der Begriff „Education 2030 Agenda“ - Bildungsagenda 2030" etabliert; dieser Begriff ist in der Tat womöglich sogar passender, als von ,dem ' (einen) Bildungsziel der SDGs zu reden, weil es sich wahrlich um eine umfassende Agenda handelt; denn allein das SDG 4 umfasst schon zehn Unterpunkte, zu denen aber noch eine Reihe von Unterpunkten der andereren 16 SDGs kommen, die etwas mit Bildung und Erziehung zu tun haben (vgl. die Übersicht in UIS, 2016, S. 16).

Die SDGs der Nachhaltigkeitsagenda sind nach dem Bekunden der UNESCO „das Ergebnis des wohl inklusivsten Konsultationsprozesses in der Geschichte der Vereinten Nationen, in den bedeutsame Beiträge aus allen Bereichen der Gesellschaft, von allen Akteuren der internationalen Gemeinschaft und aus allen Teilen der Welt eingeflossen sind. Alle Mitglied- 
staaten, das gesamte VN-System, Experten und ein Querschnitt der Zivilgesellschaft, die Wirtschaft und - dies ist besonders wichtig - Millionen Menschen aus allen Teilen der Welt haben sich zu dieser umfassenden Agenda verpflichtet, die darauf abzielt, globale Herausforderungen anzugehen und das Gemeinwohl zu fördern“ (DUK, 2017b, S. 1). Als Prinzipien dieser Bildungsagenda werden folgende herausgestellt (ebd., S. 2f.):

1. Bildung ist ein Menschenrecht.

2. Bildung ist ein öffentliches Gut.

3. Geschlechtergleichberechtigung.

Wie sind diese Prinzipien einzuordnen? In der Pädagogik ist die Vorstellung von ,Bildung als Menschenrecht' offenbar unstrittig (Lenhart, 2006, S. 155-179). Diese Maxime gilt nicht nur für die sog. Dritte Welt, sondern bietet auch Anhaltspunkte für einen kritischen Blick auf das deutsche Bildungssystem, wie der Bericht des UN-Sonderberichterstatters für das Recht auf Bildung, Vernor Muńoz-Villalobos, nach seinem Deutschland-Besuch im Jahre 2006 verdeutlicht, der in der Öffentlichkeit und Politik hohe Wellen schlug, weil er dem deutschen Schulwesen ob seiner hohen Selektivität eine Tendenz zur sozialen Diskriminierung attestierte (Overwien \& Prengel, 2007; Lohrenscheit, 2007; Overwien, 2010). Was das Thema ,Bildung als öffentliches Gut' anbetrifft, so werden in der historisch-vergleichenden Bildungsforschung inzwischen die ausgedehnten Theorieüberlegungen und empirischen Forschungen der so geannten, World Polity' oder, Weltkultur'-Theorie breit rezipiert, die von und im Umkreis des Stanforder Wissenschaftlers John W. Meyer begründet wurden. Kurz gesagt, unterstreicht dieser Ansatz die Entstehung und globale Verbreitung schulischer Bildung im Rahmen eines nationalstaatlichen Bildungssystems, d.h. als ein in der Hand öffentlicher Instanzen praktiziertes Recht auf Bildung, mit der Pflicht des Staates, dieses Recht allen seinen Bürgern zu garantieren, was sich in Begriffen wie Schulpflicht oder Pflichtschulwesen manifestiert (Meyer, 2005; Adick, 2009). Im Hinblick auf das Prinzip, Geschlechtergleichberechtigung' fragt man sich jedoch, warum es überhaupt der namentlichen Nennung dieses Prinzips bedarf, wo doch eigentlich schon im ,Menschenrecht' auf Bildung alle Geschlechter inbegriffen sein sollten, und, wenn man schon den Faktor, Gleichberechtigung' unterstreichen will, warum er dann (einzig) auf,Geschlecht' fokussiert ist und nicht andere Diskriminierungsdimensionen (wie Einkommen, Religion, Wohnort, Behinderung u.a.) nennt - zumal die übergeordnete Benennung des SDG 4 (siehe oben) von ,inklusiv" und „chancengerecht" spricht. Die Antwort darauf ergibt sich nach Meinung der Autorin, wenn man die Vorgeschichte des SDG 4 betrachtet, in der die Geschlechterfrage eine zentrale Bedeutung erhalten hatte, wie nun folgend kurz skizziert wird.

\section{Historischer Kontext der heutigen Bildungsagenda}

Die weltweiten Bildungsentwicklungen waren seit den 1990er Jahren durch folgende langfristige Programme der Vereinten Nationen verstärkt auf die internationale und die nationale politische Arena getreten (Adick, 2017a):

EFA: Education for All (1990-2015):

In diesem Programm ging es darum, Bildung für alle Menschen dieser Welt als Bürgerrecht durchzusetzen. Zwar war ,Bildung als Menschenrecht' auch schon im Artikel 26 der Menschenrechts-Charta der Vereinten Nationen von 1948 aufgeführt worden, aber hierzu gab es noch keine eigene Deklaration, bis dann im Jahr 1990 auf dem Weltbildungsforum in Jomtien/ Thailand die „Weltdeklaration ,Bildung für alle“" verabschiedet wurde, deren erster Artikel lautet: „Jede Person, ob Kind, Jugendlicher oder Erwachsener, muss in der Lage sein, Bildungschancen entsprechend ihren grundlegenden Lernbedürfnissen wahrzunehmen. Dazu gehören sowohl die wichtigsten Lernmittel (Lesen, Schreiben, mündlicher Ausdruck, Rechnen und das Lösen von Problemen) als auch grundlegende Lerninhalte (Kenntnisse, Fertigkeiten, Werte und Haltungen).“ (DUK 1991, S. 1). Diese Deklaration hatte besondere Relevanz für die Bildungskrise in vielen sog. Entwicklungsländern (Lenhart, 1993).

Die Ziele von Jomtien richteten sich auf die flächendeckende Einschulung aller Kinder, die Reduzierung der Zahl der Analphabeten und den gleichen Zugang beider Geschlechter zu Bildung und sollten bis zum Jahr 2000 erreicht werden. Als klar wurde, dass dies nicht der Fall sein würde, beschlossen die Delegierten auf dem Weltbildungsforum in Dakar/Senegal im Jahr 2000 die Fortsetzung des Programms (bis 2015) mit sechs zentralen und gegenüber den Jomtien-Formulierungen teils präziseren Zielformulierungen: (1) Ausbau der frühkindlichen Bildung, (2) weltweite Verwirklichung des Grundschulbesuchs, (3) Zugang zu weiterer (Aus-)Bildung für Jugendliche, (4) Erhöhung der Alphabetisierungsrate, besonders auch bei Frauen, um 50\%, (5) gleiche Bildungsbeteiligung beider Geschlechter im Primar- und Sekundarschulwesen bis 2005 und im gesamten Bildungswesen bis 2015, und (6) Verbesserung der Bildungsqualität. Obwohl das EFA-Programm oft vorschnell nur mit ,Bildungsproblemen der Dritten Welt' assoziiert wird, hat es sich von Anfang an um ein internationales Programm gehandelt, an dem sich praktisch alle Länder dieser Welt beteiligten, darunter auch Deutschland (Müller \& Hinzen, 2001).

\section{Millennium Development Goals (2001-2015):}

Die in Dakar formulierten EFA-Ziele decken sich teilweise mit den ebenfalls im Jahr 2000 von den Vereinten Nationen ausgerufenen Millennium Development Goals (MDGs), die ebenfalls bis 2015 erreicht werden sollten, und zwar die flächendeckende Durchsetzung kostenloser Primarschulbildung (Universal Primary Education) bis 2015 (MDG-Ziel 2) sowie die Gleichstellung der Geschlechter und die Förderung von Mädchen und Frauen (MDG-Ziel 3).

Zum Ende beider Programme (EFA und MDGs) war dann klar geworden, dass deren Ziele keinesfalls erreicht werden würden, was - so die VN-Entscheidung - die Notwendigkeit für ein neues, wiederum fünfzehn Jahre (2016 bis 2030) andauerndes Weltprogramm begründete, das nunmehr unter der übergeordneten Maxime einer ,Nachhaltigen Entwicklung' gefasst wurde, zu der auch Bildung und Erziehung in all ihren Facetten gehören, so wie es nunmehr im SDG 4 grundgelegt ist.

\section{Bildung für nachhaltige Entwicklung (BNE)- Dekade (2005-2014):}

Was das SDG 4 betrifft, so sollte nicht unerwähnt bleiben, dass auch hierfür ein Vorläuferprogramm existiert, an das erinnert werden kann, wenn es um die inhaltlich-curriculare Vermittlung der Anliegen der SDGs geht: Die UN-Dekade „Bildung 
für nachhaltige Entwicklung“ (2005-2014) (Adick, 2017b). Zwar war bereits 1990, auf der UN-Umweltkonferenz in Rio de Janeiro in die Agenda 21 (für das 21. Jahrhundert) ein eigener Bildungskatalog aufgenommen worden (Kapitel 36), in dem eine Neuorientierung der formalen wie der non-formalen Bildung, der beruflichen wie der Weiterbildung, in Richtung Nachhaltigkeit gefordert wurde. Doch erst in der eigens diesem Thema gewidmeten BNE-Dekade geschah die ausgewiesene programmatische Verknüpfung beider Anliegen: ,Bildung ' und ,Nachhaltigkeit'. Gerade in Deutschland zeichnete sich diese Dekade durch eine vergleichsweise breite staatliche wie auch zivilgesellschaftliche Beteiligung aus. An dieser Stelle sei unterstrichen, dass über die Jahre hinweg auch viele Beiträge in dieser Zeitschrift ZEP (Zeitschrift für internationale Bildungsforschung und Entwicklungspädagogik) zum Themenbereich ,BNE“ publiziert worden sind. Ferner sei daran erinnert, dass die Halbzeitkonferenz (2009) der BNE-Dekade in Bonn stattfand und diese öffentlichkeitswirksam zur Sprache brachte. Es wird daher davon ausgegangen, dass die deutschsprachige Diskussion um die inhaltlich-intentionale breite Vermittlung der SDGs in Schule, Hochschule und außerschulischen Bildungsangeboten an etliche vorliegende Konzepte aus der Ära der BNE-Dekade anknüpfen kann, insbesondere, da diese Dekade derzeit ihre Fortsetzung im Weltaktionsprogramm BNE findet.

Zusammenfassend gesagt, kann das SDG 4 also als Fortsetzung und Weiterentwicklung vorheriger UN-Programme angesehen werden, die alle praktisch zur gleichen Zeit - 2015 - ausliefen, deren Ziele aber (noch) nicht (bzw. nicht vollständig oder zufriedenstellend) erreicht worden waren, so dass sie weiterhin verfolgt werden sollten. Bei inhaltlichen Debatten sollte dieser historische Kontext daher nicht unerwähnt bleiben.

\section{Operationalisierung des SDG 4: Was soll wirklich erreicht werden?}

Die Agenda 2030 zeichnet sich, wie bei Empfehlungen allgemein bekannt, durch eine gewisse genretypische ,Schwammigkeit' aus, die aus dem Umstand resultiert, möglichst breite Akzeptanz zu erzielen - schließlich muss sie ja von praktisch allen Ländern dieser Welt erstens zumindest formal akzeptiert werden (Verabschiedung im UN-System) und zweitens, mindestens in Ansätzen national umgesetzt werden. Im Folgenden soll nach möglichst handfesten Aussagen in den zehn Unterpunkten des SDG 4 (Unterziele 4.1. bis 4.7 und Implementierungsmechanismen 4.a, 4.b, 4.c; vgl. die linke Spalte von Abb. 1) gesucht werden, um zu erkunden, was genau - operativ - im Bildungswesen erreicht werden soll; d.h. eine bildungstheoretische Diskussion der inhaltlichen Auslegung des SDG 4 ist in diesem Aufsatz nicht intendiert.

In der von der Deutschen UNESCO-Kommission verbreiteten Broschüre zur „Bildungsagenda 2030“ werden deren Unterziele in Ansätzen präzisiert. Zum ersten Unterziel (SDG 4.1) heißt es dort: „Die Gewährleistung von 12 Jahren kostenloser, öffentlich finanzierter, inklusiver, chancengerechter, hochwertiger Grundschul- und Sekundarschulbildung - wovon mindestens neun Jahre obligatorisch sein und zu relevanten Lernergebnissen führen sollen - sollte für alle ohne Diskriminierung sichergestellt werden “ (DUK, 2017a, S. 11). In der zugehörigen Fußnote (Fn 5; ebd.) heißt es: „Die ersten neun Jahre der for- malen Bildung, d.h. die Gesamtdauer von ISCED 1 und 2: ISCED 1 entspricht der Grundschule und dauert üblicherweise sechs Jahre (variiert in den Staaten zwischen vier und sieben Jahren) und ISCED 2 ist die untere Sekundarschulbildung, die typischerweise drei Jahre dauert (auch hier gibt es Unterschiede)." An dieser Stelle muss ISCED kurz erläutert werden. Das Akronym bedeutet International Standard Classification of Education; diese Klassifikation von Bildungsstufen wurde bereits 1976 erstmals von der UNESCO aufgestellt, um die Vergleichbarkeit internationaler Bildungsdaten zu gewährleisten. Nach mehreren Überarbeitungen besteht die jetzige Version (ISCED 2011) aus acht Stufen, die von der Frühkindlichen Bildung (Level 0) bis zur Promotion oder Äquivalent (Level 8) reichen; ergänzend befasst sich die ISCED-F (Classification of fields of education and training) aus dem Jahre 2013 mit beruflichen (incl. hochschulischen) Ausbildungsfeldern, darunter z.B. auch verschiedene Varianten der Lehrerbildung.

Betrachtet man dieses erste Unterziel im Kontext der historischen Entwicklung, so lässt sich eine eindeutige Tendenz zur Ausweitung der weltweit für verbindlich erachteten Schulbesuchsdauer schlussfolgern: Während 1990 in Jomtien noch über Grundbildung - formal und/oder auch non-formal? - diskutiert wurde, dieser Begriff sich dann in Dakar 2000 bzw. im MDG-Programm auf Universal Primary Education (UPE) zuspitzte, womit in der Regel sechs Jahre Schulbildung gemeint waren, so muss man nun, im SDG 4-Prozess, schon genau hinschauen und weiterlesen, wenn die Schlagzeile einer Pressemitteilung zum Beispiel „263 Millionen Kinder und Jugendliche weltweit gehen nicht zur Schule“ lautet, was sich erst aufklärt, wenn im Text dann wie folgt differenziert wird:

„263 Millionen Kinder und Jugendliche weltweit gehen nicht zur Schule. Das entspricht in etwa einem Viertel der Bevölkerung Europas. Die jüngste UNESCO-Studie „Leaving no one behind: How far on the way to universal primary and secondary education?" stellt fest: 61 Millionen Kinder im Grundschulalter (6-11 Jahre), 60 Millionen im unteren Sekundarschulalter (12-14 Jahre) und 142 Millionen im oberen Sekundarschulalter (15-17 Jahre) haben keinen Zugang zur Schule. Mit der globalen Nachhaltigkeitsagenda hat die Weltgemeinschaft 2015 beschlossen, bis 2030 eine inklusive, chancengerechte und hochwertige Bildung für alle sicherzustellen. (Pressemitteilung der DUK vom 14.07.2016)

Für viele Länder, die unter den EFA- und MDG-Zielen bis 2015 schon Schwierigkeiten mit der Erreichung von UPE hatten, mag es wie eine unerreichbare Messlatte erscheinen, wenn ihnen nun ,Universal Primary and Secondary Education als Ziel vorgesetzt wird - ein Ziel, das sie ganz sicher nur mit massiver externer ,Bildungshilfe erreichen können, was in der Regel mehr oder weniger explizit auch ,externe Einflussnahme‘ und Abhängigkeit bedeutet.

Im zweiten Unterziel (SDG 4.2) ist von einem kostenlosen und verpflichtenden Vorschuljahr mit gut ausgebildeten Pädagoginnen und Pädagogen die Rede (ebd., S. 12). Das macht aus den zwölf Jahren Schulbildung im Endeffekt dann sogar 13 Jahre. Allerdings muss man genau lesen, denn das eine Vorschuljahr (kostenfrei und obligatorisch) einzuführen wird nur „angeregt“ (ebd.); d.h. von Gewährleistung oder Sicherstellung ist nicht die Rede. Es macht aber einen großen Unterschied, ob ein Staat mit der Tradition eines freiwilligen ,Kinder- 
Table 1. Global education targets and indicators for the 2030 Agenda for Sustainable Development

\section{- Goal 4. Ensure inclusive and equitable quality education and promote lifelong learning} opportunities for all

Targets Indicators

4.1 By 2030 ensure that all girls and boys complete free, equitable and quality primay and secondary education leading to relevant and effective learning outcomes.

4.2 By 2030 , ensure that all girls and boys have access to quality early childhood development, care and pre-primary education so that they are ready for primary education
4.1.1 Proportion of children and young people: (a) in Grade 2 or 3; (b) at the end of primary education; and (c) at the end of lower secondary education achieving at least a minimum proficiency level in (i) reading and (ii) mathematics, by sex

4.2.1 Proportion of children under 5 years of age who are developmentally on track in health, learning and psychosocial well-being, by sex

4.2.2 Participation rate in organized learning (one year before the official primary entry age), by sex

4.3 By 2030, ensure equal access for all women and men to affordable and quality technical, vocational and tertiary education, including university

4.4 By 2030 , substantially increase the number of youth and adults who have relevant skills, including technical and vocational skills, for employment, decent jobs and entrepreneurship

4.5 By 2030, eliminate gender disparities in education and ensure equal access to all levels of education and vocational training for the vulnerable, including persons with disabilities, indigenous peoples and children in vulnerable situations

4.3.1 Participation rate of youth and adults in formal and non-formal education and training in the previous 12 months, by sex

4.4.1 Proportion of youth and adults with information and communications technology (ICT) skills, by type of skill

4.5.1 Parity indices (female/male, rural/urban, bottom/ top wealth quintile and others such as disability status, indigenous peoples and conflict-affected, as data become available) for all education indicators on this list that can be disaggregated

4.6 By 2030 , ensure that all youth and a substantial proportion of adults, both men and women, achieve literacy and numeracy

4.6.1 Percentage of population in a given age group achieving at least a fixed level of proficiency in functional (a) literacy and (b) numeracy skills, by sex

4.7 By 2030, ensure that all learners acquire the knowledge and skills needed to promote sustainable development, including, among others, through education for sustainable development and sustainable lifestyles, human rights, gender equality, promotion of a culture of peace and non-violence, global citizenship and appreciation of cultural diversity education and of culture's contribution to sustainable development

4.7.1 Extent to which (i) global citizenship education and (ii) education for sustainable development, including gender equality and human rights, are mainstreamed at all levels in: (a) national education policies, (b) curricula, (c) teacher education and (d) student assessment 4.a Build and upgrade education facilities that are child, disability and gender sensitive and provide safe, non-violent, inclusive and effective learning environments for all

4.a.1 Proportion of schools with access to: (a) electricity; (b) the Internet for pedagogical purposes; (c) computers for pedagogical purposes; (d) adapted infrastructure and materials for students with disabilities; (e) basic drinking water; (f) single-sex basic sanitation facilities; and $(\mathrm{g}$ ) basic handwashing facilities (as per the WASH indicator definitions)

4.b By 2020, substantially expand globally the number of scholarships available to developing countries, in particular least-developed countries, small island developing States and African countries, for enrolment in higher education, including vocational training and information and communications technology, technical, engineering and scientific programmes, in developed countries and other developing countries

4.c By 2030 , substantially increase the supply of qualified teachers, including through international cooperation for teacher training in developing countries, especially least-developed countries and small island developing States 4.b.1 Volume of official development assistance flows for scholarships by sector and type of study

Quelle: UIS (2016): Laying the foundation to measure SDG4 (Sustainable development data digest 2016), Montreal/Canada, S. 28;

verfügbar unter http://uis.unesco.org/sites/default/files/documents/laying-the-foundation-to-measure-sdg4-sustainable-development-data-digest-2016-en.pdf; Zugriff am 10.01.2018 
garten'-Besuchs, wie z.B. Deutschland, nunmehr Vorschuleinrichtungen samt zugehöriger Ausbildung von pädagogischem Personal gewährleisten muss und folglich im weltweiten Monitoring daran gemessen wird), oder ob dies nur empfohlen wird. In der ISCED-F gibt es u.a. die Kategorie „Training for pre-school teachers“, die erstens darauf hinweist, dass es dies in Ländern bereits gibt, und zweitens, dass die dort Tätigen als ,Lehrpersonen' klassifiziert werden und nicht etwa ,Kindergärtnerinnen' sind - was enorme Auswirkungen auf die Gestaltung von Lehramtsausbildungssystemen hat.

Im dritten Unterziel (SDG 4.3) sieht man einen regelrechten ,Eiertanz' zwischen beruflicher und akademischer Bildung (ebd., S. 13f.). Es fällt auf, dass hier der Hochschulbereich erstmals (im Vergleich zum EFA-Prozess) explizit in einem eigenen Bildungsziel fokussiert wird. Aber auch hier muss man genau lesen, da die Terminologie allerlei Fallstricke bereit hält. Es heißt dazu: „Die Bereitstellung von tertiärer Bildung sollte schrittweise kostenlos werden, in Übereinstimmung mit bestehenden internationalen Übereinkommen" (ebd., S. 14). Eine Fußnote (Fn 6; ebd.) lautet erklärend: „Die tertiäre Bildung baut auf der Sekundarschulbildung auf und bietet Lernaktivitäten in spezialisierten Bereichen. Ihr Ziel ist Lernen mit einem hohen Grad an Komplexität und Spezialisierung. Die tertiäre Bildungsstufe schließt ein, was allgemein unter akademischer Bildung verstanden wird, aber auch fortgeschrittene berufliche Bildung und Weiterbildung. Sie

ein Anspruch auf kostenfreie tertiäre Bildung (inklusive keine Studiengebühren an Hochschulen) besteht nicht.

Die Unterziele SDG 4.4 bis SDG 4.6 sind noch allgemeiner formuliert als die bisher erläuterten und bieten insbesondere im Vergleich zum EFA-Programm wenig konkret Neues. Es geht darin z.B. um die Sicherstellung von Lese-, Schreib- und Rechenfähigkeiten (Literacy) aller Erwachsenen oder die Geschlechtergerechtigkeit auf allen Bildungsstufen. Hervorzuheben ist allerdings die explizit geforderte inhaltlich-curriculare Ausrichtung aller (d.h. jeglicher) Bildung auf ,Bildung für nachhaltige Entwicklung' (BNE) sowie auf,Global Citizenship Education' (GCED) in einem eigenen Unterziel (SDG 4.7). Es ist noch nicht abzusehen, welche Auswirkungen dieses Postulat auf Schule, Hochschule und außerschulische Bildung weltweit haben wird, ob es z.B. jemals irgendwo zu einem entsprechenden neuen eigenständigen Unterrichtsfach kommen wird, oder - was wahrscheinlicher ist - ob BNE und GCED in bestehende Curricula integriert werden, wie dies z.B. in Deutschland seit Jahrzehnten mit anderen Anliegen des, Globalen Lernens' wie Friedenserziehung, entwicklungspolitische Bildung oder Menschenrechtserziehung der Fall ist, die über den Modus von Empfehlungen der Kultusministerkonferenz (KMK) als sog. Querschnittthemen in den Unterricht eingefügt werden (Adick, 2008, S. 93-99). Nach Meinung der Autorin muss auch geklärt werden, in welchem Verhältnis BNE und GCED zueinander und zu den bereits bestehenden Konzepten stehen. Ist GCED beispielsweise als eine neue Variante im Gemenge des ,Globalen Lernens' anzusehen? Hierüber könnte ein Blick in ein einschlägiges, dem ,Globalen Lernen' gewidmetes Lexikon Aufschluss geben (Lang-Woijtasik \& Klemm, 2017). Oder bietet es sich im Gegenteil sogar an, GCED gerade im weltweiten Diskurs als eine neue übergeordnete Klammer aller diversen ,interkulturellen und internationalen Pädagogiken' (Adick, 2008, S. 116-130) anzusehen, wie es die Autorin nahelegen würde? In jedem Fall aber müssen dabei alle SDGs (und nicht nur das hier im Mittelpunkt stehende SDG 4) zum Gegenstand von Bildungspolitik und Unterricht gemacht werden, wie dies etwa in Vorschlägen der von Marco Rieck-

umfasst die ISCED Levels 5 (Short-cycle tertiary education), 6 (Bachelor's degree or equivalent), 7 (Master's degree or equivalent) und 8 (Doctorate or equivalent). Die Bildungsinhalte im Tertiärbereich sind komplexer und fortgeschrittener als auf den unteren Levels." An ,hard facts' bleibt: Was nach der (min.) 12 Jahre Schulbildung plus gewünscht 1 Jahr Vorschule kommt, nennt sich ,tertiäre Bildung', ist aber keinesfalls zu verwechseln mit ,Hochschulbildung ' oder gar ,Universität‘. Obligatorisch ist hier nichts; Zugangsbarrieren sollen (nur) verringert werden; mann als leitendem Autor betreuten Broschüre zu den ,Learning Objectives' der SDGs exemplarisch dargelegt wird (UNESCO, 2017, bes. Kap. 2, S. 47ff.).

Die drei ,Implementierungsmechanismen' (SDG 4.a SDG 4.c) formulieren Grundsätze, wie die genannten sieben Unterziele operativ umgesetzt werden sollen. An konkreten Forderungen finden sich die Erhöhung der Stipendien für Studierende insbesondere aus besonders benachteiligten ,Entwicklungsländern` sowie der Verweis auf den Ausbau der Lehramts- 
ausbildung angesichts der avisierten Bildungsexpansion. So wird etwa prognostiziert, für die universale Grundschulbildung bis 2030 benötige man 3,2 Mio. und für die untere Sekundarschulbildung weitere 5,1 Mio. Lehrpersonen zusätzlich; hinzu kämen die Lehrpersonen, die zwischen 2015 und 2030 aus dem Beruf ausscheiden; ferner arbeiteten in vielen Ländern noch Lehrpersonen, die gar keine den nationalen Standards entsprechende Lehramtsausbildung durchlaufen haben (DUK, 2017a, S. 24). Hier ergeben sich viele Herausforderungen für die ,Bildungshilfe im Rahmen der Entwicklungszusammenarbeit sowie - insbesondere in der sog. Dritten Welt - für die nationale Bildungsplanung vor allem hinsichtlich der Lehrerbildung, was an dieser Stelle jedoch nicht mehr weiter thematisiert werden kann.

\section{Monitoring: Wie werden die Fortschritte der Bildungsagenda 2030 gemessen?}

Während des EFA-Prozesses (nach Dakar 2000) begann die Epoche der umfangreichen, mit Bildungsdaten aus möglichst allen Ländern dieser Welt gestützten, globalen Bildungsberichterstattung namens (EFA) Global Monitoring Reports (GMRs, von 2002 bis 2015). Seit 2016 firmiert die Berichterstattung über das SDG 4 fast titelgleich unter (New) Global Education Monitoring Reports (GEMs); jeweils mit Kurzfassungen in verschiedenen Sprachen dieser Welt, so auch in Deutsch, und kostenlos im Internet für Interessierte aus Politik, Medien, Zivilgesellschaft und Wissenschaft zugänglich. Neben der Aufklärung der Weltöffentlichkeit wird Handlungsbedarf signalisiert und für den Fortgang der Nachhaltigkeitsagenda geworben, wie es sich z.B. an folgendem Zitat ablesen lässt:

„Der Weltbildungsbericht 2016 prognostiziert die Erfolgsaussichten zur Erreichung universeller Sekundarschulbildung bis 2030 unter Verwendung globaler repräsentativer Datensätze und einer komplexen Methodologie. Das Ergebnis ist ernüchternd: Die Welt wird ihre globalen Bildungsverpflichtungen mit 50 Jahren Verspätung erfüllen. Bei anhaltenden Trends werden erst im Jahr 2042 alle Kinder weltweit eine Grundschulbildung abschließen. Berechnungen zufolge wird eine universelle untere Sekundarschulbildung (in Deutschland Abschluss Sekundarbereich I) erst 2059 erreicht, eine universelle obere Sekundarschulbildung (in Deutschland Fach-/Hochschulreife oder abgeschlossene Lehrausbildung) erst bis zum Jahr 2084. Die ärmsten Länder werden Grundschulbildung für alle Kinder über 100 Jahre später verwirklichen als die reichsten. Die wichtigste Schlussfolgerung lautet, dass sowohl Länder mit niedrigem als auch mit mittlerem Einkommen enorme Anstrengungen unternehmen müssen, um die aktuellen Trends umzukehren und das Unterziel 4.1 zu erreichen" (DUK, 2016, S. 25).

Im Zuge des SDG 4-Monitorings werden riesige Datenmengen gesammelt und ausgewertet (,big data'). Hierfür ist das UIS (UNESCO-Institute for Statistics) in Montreal/Kanada zuständig, das auf seiner Webseite einen extra Link „Sustainable Development Goal 4“ ausweist, dessen Eingangsseite mit folgendem Statement beginnt:

„With the adoption of the Sustainable Development Goals (SDGs), the UIS has been clearly recognised as ,the official source of cross-nationally comparable data on education', as confirmed in the Education 2030 Framework for Action. The UIS has been given the mandate to ,work with partners to develop new indicators, statistical approaches and monitoring tools to better assess progress across the targets related to UNESCO's mandate (...) “" [http://uis.unesco.org/en/topic/sustainabledevelopment-goal-4; Abruf am 05.03.2018]

Hierzu stellt das UIS die Publikationsreihe „SDG 4 Data Digest" bereit, in der es um die Operationalisierung der Ziele zum Zwecke der Datengewinnung und ihrer Auswertung geht, mit derzeit (d.h. zur Zeit der Abfassung dieses Beitrages) folgenden Bänden:

„Laying the foundation to measure SDG4. Sustainable development data digest 2016“ (UIS, 2016). Mit einem kursorischen vergleichenden Blick auf andere Organisationen aus dem VN-System beansprucht das UIS in dieser Veröffentlichung, Lieferant eines ,Gold Standards' der weltweiten Bildungsstatistik zu sein: „The UIS and other UN statistical agencies, through various bodies, set measurement norms and recommendations which are formally adopted by countries. For example, the World Health Organization's (WHO) concept of live birth or the International Labour Organization's (ILO) definition of child labour, reached by consensus among countries, are considered to be the gold standard and are integrated into national measurement systems. Likewise in the areas of its mandate, countries look to UNESCO as the authoritative source for good practices in the area of statistics" (ebd., S. 21). Ferner findet sich hier ein konziser tabellarischer Überblick sowohl über die zehn Unterziele des SDG 4 als auch über die Indikatoren zu ihrer Messung; was der Anlass dafür war, diese Tabelle hier abzudrucken (vgl. Tab. 1).

„The Quality Factor: Strengthening National Data to Monitor Sustainable Development Goal 4. SDG 4 Data Digest 2017“(UIS, 2017). In dieser Broschüre geht es darum, kohärente Standards für nationale Bildungsstatistiken vorzugeben, um diese kompatibel zu den Erfordernissen des SDG 4-Monitoring zu machen. Es wurde nämlich, so wird mitgeteilt, festgestellt, dass nicht alle Länder entsprechende Daten bereitstellen (können). Daher sollen alle nationalen Bildungsverwaltungen, falls nötig mit mehr oder weniger Anleitung des UIS, eine Strategie zur (Fort-)Entwicklung ihrer Bildungsstatistik entwickeln: "Given the complexity of the required SDG 4 data, and the need to improve their availability and quality, the implementation of a quality driven data framework to monitor SDG 4 will present a challenge for countries at all levels of development. The UIS is fully engaged in strengthening national statistical capacities through an inclusive approach, articulated around the mobilisation of national commitment, donor support as well as national and regional partnerships." (ebd., S. 42). Die Veröffentlichung definiert in einem eigenen Kapitel (ebd., Kap. 3) detaillierte Ablaufschritte für eine solche National Strategy for the Development of Education Statistics.

Da das Monitoring auch auf Erfolgsmessungen aus vorhandenen großformatigen Leistungstests (wie z.B. PISA) zurückgreifen möchte, hat das UIS einen ,Learning Assessment Capacity Index (LACI) ' kreiert; dieser zeigt ,the extent to which countries are ready to measure learning“" Wer sich schnell und komfortabel über weltweite Bildungsdaten informieren will, findet mit dem ,eAtlas for Education 2030` das richtige Werkzeug dazu. Das UIS verspricht, dass in diesem Link die verfügbaren Daten zu den globalen und thematischen Indikatoren eingestellt und laufend auf den neuesten Stand gebracht werden. Somit ist es möglich, vorliegende Daten und Übersichten 
zu jedem der zehn Unterpunkte des SDG 4 aufzurufen. Die Informationen werden übersichtlich und handlich in ExcelTabellen und Weltkarten zur Verfügung gestellt [http://uis. unesco.org/en/topic/sustainable-development-goal-4; darunter Links zum e-Atlas und zu LACI; Abruf 05.03.2018].

Insgesamt betrachtet zeigt sich somit über die Jahrzehnte seit dem Startschuss in Jomtien (1990) eine deutliche Steigerung des Anspruchs und der Finesse, mit der die UNESCO weltweite Bildungsdaten sammelt und auswertet. Laut der für die UNESCO erstellten Experten-Empfehlung „Bildung überdenken - ein globales Gemeingut?“ (engl. Original „Rethinking Education: Towards a global common good?“, 2015) verbirgt sich dahinter eine wahrhafte "Datenrevolution“ im gesamten UN-System im Zuge der post-2015-Nachhaltigkeitsstrategie - mit folgender Begründung:

„Die Erfahrungen, die seit dem Jahr 2000 mit globalen Zielvorgaben im Rahmen der MDG- und EFA-Erfahrungen gemacht wurden, haben zur Berichterstattung über aggregierte, nationale Daten angeregt. Allerdings blieb dabei das Ausmass landesinterner Ungleichheiten und Disparitäten meist verborgen. Wenn uns bei der Bereitstellung effektiver und relevanter Lernangebote für alle die Chancengleichheit am Herzen liegt, dann sollten die nationalen Zielvorgaben eine Berichterstattung über weit stärker disaggregierte Daten erlauben. Die Datenerfassung muss neben herkömmlichen Diskriminierungsfaktoren wie Geschlecht und Stadt-Land-Gefälle außerdem Einkommen und, sofern möglich, Minderheitenstatus berücksichtigen. Dies kann durch eine bessere Nutzung von alternativem Datenmaterial beispielsweise aus Haushaltsumfragen zu Lebensstandard, Gesundheit und Erwerbstätigkeit erfolgen." (Schweizerische UNESCO-Kommission, 2016, S. 72).

Anders ausgedrückt: Die nationalen Bildungsstatistiken, wie sie bisher an die UNESCO gemeldet werden, kaschieren oftmals mehr als dass sie klare faktengestützte Aussagen erlauben. Daher muss die UNESCO über ihr Statistikinstitut einen (quasi-)eigenständigen Zugriff auf nationale Daten erhalten, die zur Messung ihrer Indikatoren notwendig sind. Vor diesem Hintergrund ist es auch nicht verwunderlich, dass das UIS unter dem Titel ,capacity building' detailliert bis hin zu normierten Datenerfassungsvorlagen vorgibt, wie die aus der obigen Tabelle (Tab. 1) ersichtlichen 43 empirischen Indikatoren der Bildungsagenda 2030 ,richtig' erhoben werden müssen (UIS, 2017, Tab. 2, S. 47). Damit wandelt sich in der Tat die Rolle der von der UNESCO geführten Bildungsstatistik von der einer Sammlung und Aufbereitung von nationalen Bildungsdaten zu der einer extern dirigierten Erhebung und Verarbeitung nationaler Bildungsdaten. Zugegebenermaßen geschieht dies im Interesse an einer Vergleichbarkeit der Datenerhebung und Qualitätssicherung des Monitoring; der weitere Fortgang der SDG 4 Berichterstattung wird zeigen, ob und in welcher Weise sich (alle?) Regierungen diesem UIS-Datenregime unterwerfen oder eher weniger kooperativ damit umgehen.

\section{Abschließende Bemerkungen zur Relevanz des SDG 4 für die internationale Bildungsforschung}

Die globale Bildungsagenda 2030 kann unter verschiedenen Perspektiven thematisiert werden, z.B.: Umsetzung auf glo- baler Ebene, inhaltliche Auslegung von Bildung (,Bildungsphilosophie'), Relevanz für die (eigene) nationale Bildungspolitik oder Implikationen für die praktische Entwicklungszusammenarbeit. Im hier vorliegenden Aufsatz wurden diese Themen nicht vertieft; stattdessen wurde gefragt: Welche Bedeutung haben das SDG 4 und seine Berichterstattung für die internationale Bildungsforschung? Gerade der Punkt der Forschung ist bedeutsam, da im Zuge des SDG 4-Monitoring riesige Datenmengen gesammelt werden, die einzelne Forschende gar nicht zuwege bringen könnten, die nun aber für eigene Forschungsinteressen, z.B. zur Überprüfung von Hypothesen im Rahmen von Sekundäranalysen, bereit stehen. Insofern ist ein Blick auf das Zustandekommen dieser Daten, wie es in diesem Aufsatz angedeutet wurde, unerlässlich.

Auf die zum SDG 4 aufbereiteten UIS-Daten kann und wird vermutlich in Zukunft in kleinen oder größeren Forschungsarbeiten zurückgegriffen werden. Die Zeit, in der man in der internationalen Bildungsforschung bei Recherchen zu weniger breit akademisch bearbeiteten Ländern (z.B. kleine Länder oder solche aus der sog. Dritten Welt) fast schon pauschal und ungeprüft ,fehlende Daten' beklagen konnte, sind eindeutig vorbei. Der barrierefreie Zugriff auf die UIS-Daten gestattet es nun, auch Fragestellungen zu bearbeiten, die in früheren Jahrzehnten aufwändige Recherchen bis hin zu kostspieligen Auslandsaufenthalten erforderlich gemacht hätten und daher womöglich gar nicht erst in Angriff genommen wurden. Wie konnte vor dreißig, vierzig Jahren z.B. eine Masteroder Doktorarbeit über ein Thema wie ,Regionale, schichtund/oder geschlechtsspezifische Determinanten von Schulbesuch und Schulleistungen' im Lande X realisiert werden? Während man in Westeuropa vielleicht noch relativ problemlos Daten in Publikationen finden oder nationale Statistikämter kontaktieren konnte, waren außerhalb der ,westlichen Welt entsprechende Datensätze kaum zu erhalten. Also mussten sie vor Ort erhoben werden, wozu umfangreiche Vorarbeiten erforderlich waren wie Landeskontakte herstellen, Förderprogramme eruieren, Forschungsgenehmigung und Visum beantragen, die Landessprache hinreichend beherrschen und anderes mehr. Die UIS-Daten sind zwar noch lange nicht komplett für alle Länder und Indikatoren vorhanden, aber die Rubriken füllen sich von Jahr zu Jahr und gestatten es, viele Forschungsfragen $\mathrm{zu}$ realisieren. Die Tatsache, dass nun so problemlos im Prinzip auf Bildungsdaten aller Länder zurückgegriffen werden kann, birgt jedoch auch die Gefahr, sich eventuell zu bequem auf die vorliegenden Daten zu verlassen und diese exzessiv für Analysen zu verwenden, ohne z.B. die gewählten Indikatoren nochmals zu hinterfragen oder eigene Forschungsanstrengungen zu unternehmen, etwa für qualitative Fallstudien, die die quantitativen Marker ergänzen, oder ähnliches.

Auch in der hochschulischen Lehre kann das Thema „SDG 4“ in vielfältiger Weise herangezogen werden, z.B. in Seminaren zum Thema ,Internationale Bildungspolitik', indem auf die Relevanz des UN-Systems, vor allem der UNESCO, für regionale (z.B. Europa) und nationale Bildungspolitik eingegangen wird. Auch in den (von Studierenden der Pädagogik oft eher als ,trocken' empfundenen) Veranstaltungen zu empirischen Forschungsmethoden bietet sich eine Befassung mit dem SDG 4-Monitoring an, indem z.B. auf die Problematik 
des Vergleichs im globalen Kontext eingegangen wird oder Möglichkeiten der Nutzung der Daten für die Zwecke von Sekundäranalysen erläutert werden. Studierende mögen sich im eAtlas for Education 2030 zum Beispiel für Seminar- oder Examensarbeiten Bildungsdaten zu Ländern holen, über deren Bildungswesen nur wenige akademische Publikationen und dann vielleicht nur solche ohne aktuelle Bildungsdaten vorliegen. Oder studentische Arbeitsgruppen können in einer online-Seminarsitzung arbeitsteilig zu verschiedenen Ländern gezielt vorher definierte Daten suchen und anschließend im Plenum vergleichen. Auf diese Weise mag vielleicht auch die Motivation entstehen, sich im Laufe des Studiums, daneben auch zivilgesellschaftlich oder ehrenamtlich, und ggfs. später auch beruflich für Bildungsentwicklungen weltweit zu interessieren und in solchen Feldern zu forschen und zu handeln.

\section{Literatur}

Adick, C. (2008). Vergleichende Erziehungswissenschaft. Eine Einführung. Stuttgart: Kohlhammer Verlag.

Adick, C. (2009). World Polity - ein Forschungsprogramm und Theorierahmen zur Erklärung weltweiter Bildungsentwicklungen. In S. Koch \& M. Schemmann (Hrsg.), Neo-Institutionalismus in der Erziehungswissenschaft. Grundlegende Texte und empirische Studien (S. 258-291). Wiesbaden: VS. Verlag für Sozialwissenschaften.

Adick, C. (2017a). Bildung für alle / Grundbildung. In G. Lang-Wojtasik \& U. Klemm (Hrsg.), Handlexikon Globales Lernen. 2. überarbeitete und erweiterte Auflage (S. 26-30). Ulm: Klemm+Oelschläger.

Adick, C. (2017b): UN-Dekade (2005-2014) Bildung für nachhaltige Entwicklung (BNE). In G. Lang-Wojtasik \& U. Klemm (Hrsg.), Handlexikon Globales Lernen. (S. 387-390), 2. überarbeitete und erweiterte Auflage. Ulm: Klemm+Oelschläger.

DUK (Deutsche UNESCO-Kommission) (1991). Weltdeklaration „Bildung für alle“ und Aktionsrahmen zur Befriedigung der grundlegenden Lernbedürfnisse, Jomtien, Thailand, 5. bis 9. März 1990. Bonn: Deutsche UNESCO-Kommission.

DUK (Deutsche UNESCO-Kommission) (2016). Bildung für Mensch und Erde: Eine nachhaltige Zukunft für alle schaffen. Weltbildungsbericht - deutsche Kurzfassung. Bonn: Deutsche UNESCO-Kommission.

DUK (Deutsche UNESCO-Kommission) (2017a). Bildungsagenda 2030. Aktionsrahmen für die Umsetzung von Sustainable Development Goal 4. Bonn: Deutsche UNESCO-Kommission.
DUK (Deutsche UNESCO-Kommission) (2017b). Unpacking SDG4. Fragen und Antworten zur Bildungsagenda 2030. Bonn: Deutsche UNESCO-Kommission.

Lang-Wojtasik, G. \& Klemm, U. (Hrsg.) (2017). Handlexikon Globales Lernen. 2. überarbeitete und erweiterte Auflage. Ulm: Klemm+Oelschläger.

Lenhart, V. (1993). Bildung für alle. Zur Bildungskrise in der Dritten Welt. Darmstadt: WBG (Wissenschaftliche Buchgesellschaft).

Lenhart, V. unter Mitarbeit von Druba, V. und Batarilo, K. (2006). Pädagogik der Menschenrechte, 2. überarbeitete und aktualisierte Auflage, Wiesbaden: VS.

Lohrenscheit, C. (2007). Das Menschenrecht auf Bildung und seine Umsetzung in Deutschland: Zum Besuch des Sonderberichterstatters der Vereinten Nationen in Deutschland. In Politisches Lernen, 2007(3-4), 26-31.

Meyer, J. W. (2005). Weltkultur. Wie die westlichen Prinzipien die Welt durchdringen, hrsg. von Georg Krücken, Frankfurt: Suhrkamp.

Müller, J. \& Hinzen, H. (Hrsg.) (2001). Bildung für alle - lebenslang und lebenswichtig. Bonn: Deutscher Volkshochschulverband.

Overwien, B. (2010). Bildung für alle in Deutschland. Die bleibende Vision der Verteilung gleicher Chancen. In Zeitschrift für internationale Bildungsforschung und Entwicklungspädagogik,2010(3), 32-34.

Overwien, B. \& Prengel, A. (Hrsg.) (2007). Recht auf Bildung. Zum Besuch des Sonderberichterstatters der Vereinten Nationen in Deutschland. Opladen: Budrich.

Schweizerische UNESCO-Kommission (2016). Bildung überdenken - ein globales Gemeingut (deutsche Version von: Rethinking Education: Towards a globale common good? UNESCO 2015), Bern: Schweizerische UNESCO-Kommission.

UNESCO Institute for Statistics (2016). Laying the foundation to measure SDG4 (Sustainable development data digest 2016) Zugriff am 10.01 .2018 http://uis. unesco.org/sites/default/files/documents/laying-the-foundation-to-measure-sdg4sustainable-development-data-digest-2016-en.pdf

UNESCO Institute for Statistics (2017): The Quality Factor: Strengthening National Data to Monitor Sustainable Development Goal 4. Zugriff am 10.01.2018 http://uis. unesco.org/sites/default/files/documents/quality-factor-strengthening-nationaldata-2017-en.pdf

UNESCO (2017). Education for Sustainable Development Goals. Learning Objectives. Paris: UNESCO.

\section{Prof. Dr. Christel Adick,}

Promotion (Dr. phil.) und Habilitation in Erziehungswissenschaft; ab 1993 Lehrstuhlinhaberin für Vergleichende Erziehungswissenschaft am Institut für Erziehungswissenschaft der Ruhr-Universität Bochum (seit 08/2013 im Ruhestand). Arbeitsschwerpunkte: Historisch-vergleichende Bildungsforschung, Theorien zu Bildung in der Weltgesellschaft, Bildung und Kolonialismus (regionale Schwerpunkte: Afrika und Karibik), Globales Lernen. 


\section{Georg Müller-Christ / Bror Giesenbauer / Merle Katrin Tegeler}

\section{Die Umsetzung der SDGs im deutschen Bildungssystem - Studie im Auftrag des Rats für Nachhaltige Entwicklung der Bundesregierung ${ }^{1}$}

\begin{abstract}
Zusammenfassung
Gut zwei Jahre nach Verkündigung der UN Agenda 2030 und der 17 Sustainable Development Goals (SDGs) gibt die Studie einen ersten Überblick, inwiefern die SDGs im deutschen Bildungssystem angekommen sind. Auf Basis von Experteninterviews und Online-Recherchen werden sechs Bildungsbereiche einzeln beleuchtet: Frühkindliche Bildung, Schule, berufliche Bildung, Hochschule, non-formales und informelles Lernen sowie Fort- und Weiterbildung. Bezogen auf die Größe des deutschen Bildungsraumes mit seinen Tausenden von Bildungseinrichtungen auf den verschiedenen Qualifikationsniveaus, lässt sich feststellen, dass die SDGs bislang nur vereinzelt wahrgenommen, kommuniziert und implementiert werden. Die SDGs werden hauptsächlich dort transportiert, wo Themen wie Umweltund Naturbildung, Globales Lernen oder Bildung für nachhaltige Entwicklung schon Pfade angelegt haben. Gleichwohl scheint der Ordnungsrahmen der SDGs den bereits Aktiven Schwung mitzugeben, um an der Umsetzung von nachhaltiger Entwicklung weiterzuarbeiten und ihre Aktivitäten ansprechend darzustellen. Der Beitrag schließt mit einer Übersicht von Handlungsempfehlungen auf der Ebene der Bildungseinrichtungen sowie der Politik. Zum jetzigen Stand kommt es vor allem darauf an, die Nachhaltigkeitsziele in Curricula einzubauen und mit den Fachdisziplinen zu verknüpfen. Dies stellt besonders in den stärker selbstverwalteten Bildungsbereichen eine große Herausforderung dar und ist sowohl abhängig von politischer Unterstützung als auch vom individuellen Engagement beteiligter Akteurinnen und Akteure.
\end{abstract}

Schlüsselworte: Sustainable Development Goals, Bildung für nachhaltige Entwicklung, Bildungssystem, Curricula, Bildungspolitik

\footnotetext{
Abstract

About two years after the United Nations adopted the Agenda 2030 resolution and the 17 sustainable development goals (SDGs), the present study gives an overview of the integration of the SDGs in the German educational system. Based on expert interviews and online research, six education sectors are highlight-
}

ed individually: early childhood education, school education, higher education, vocational education, non-formal and informal education as well as vocational training and continuing education. Compared with the sheer size of the educational sector consisting of thousands of institutions on all educational levels, the SDGs have hardly been perceived, communicated and implemented. The SDGs are generally only being championed by institutions already working on related topics such as environmental education, global education, or education for sustainable development. However, the SDG framework seems to give fresh impetus to the promotion of sustainable development and helps to frame existing initiatives in new appealing ways. The contribution closes with an overview of recommendations for action both from an education policy and an institutional perspective. At the moment the main field of action is to integrate the SDGs into existing curricula and to connect them to established disciplines. This will especially challenge the more selfgoverned education sectors and is both dependent on political support and on individual commitment.

Keywords: sustainable development goals, education for sustainable development, educational policy, curricula, education system

\section{Das Anliegen der Studie}

Mit der UN-Agenda 2030 haben sich 2015 erstmalig die Nationen dieser Welt auf ein Zielsystem für eine nachhaltigere Entwicklung der Welt geeinigt: die 17 Sustainable Development Goals (SDGs). Es bleiben noch ca. zwölf Jahre Zeit, um viele dieser Ziele zu erreichen, die aus einer bislang in entwickelte und unterentwickelte Länder geteilte Welt eine Welt von Ländern mit unterschiedlichen Entwicklungsaufgaben macht. In der Neuauflage der Deutschen Nachhaltigkeitsstrategie (Bundesregierung, 2017) bekennt sich deshalb die Bundesregierung auch zur Agenda 2030 und unterstützt zudem die Umsetzung des Nationalen Aktionsplans Bildung für eine nachhaltige Entwicklung, der im Rahmen des Weltaktionsprogramms Bildung für nachhaltige Entwicklung in einem expertengestützten Diskurs 2017 entstanden ist. 
Besonders zentral für die Erreichung aller SDGs ist das SDG 4, hochwertige Bildung. Im Rahmen der vorliegenden Studie wurde im Auftrag des Rats für Nachhaltige Entwicklung (RNE) der Bundesregierung die Frage untersucht, wie der Referenzrahmen der SDGs in den unterschiedlichen Bildungsbereichen angekommen ist. Die Studie reflektiert somit die Art der Umsetzung zwei Jahre nach Verkündigung der Ziele und veranschaulicht mittels Good Practices das Engagementpotenzial in den verschiedenen Bildungsbereichen in Deutschland. Die Autor/inn/en dieser Kurzstudie, die zwischen September und Oktober 2017 durchgeführt wurde, beziehen ihr Vorwissen aus dem Engagement für mehr Nachhaltigkeit im Hochschulsystem, welches sich aus zwei Quellen speist: Zum einen sind sie im BMBF-geförderten Projekt HOCH-N tätig, einem Verbundprojekt von elf Hochschulen zur Entwicklung von Leitfäden für mehr Nachhaltigkeit in Forschung, Lehre, Betrieb und Governance von Hochschulen (www.hochn.org). Zum anderen sind sie eng verbunden mit den expertengestützten und vom BMBF geführten Diskursen und Aktionen zur Umsetzung des Weltaktionsprogramms Bildung für nachhaltige Entwicklung (www.bne-portal.de).

Die Informationen in dieser Studie entstammen einerseits aus diesem Erfahrungsraum und andererseits aus Internetrecherchen, einer kurzen Onlinebefragung sowie aus Interviews mit einzelnen Expert/inn/en aus den verschiedenen Bildungsbereichen, die in den Fachforen zur Erstellung des Nationalen Aktionsplans zur Umsetzung des Weltaktionsprogramms Bildung für nachhaltige Entwicklung tätig sind.

\section{Die Sustainable Development Goals der UN-Agenda 2030}

Mit den 17 Zielen für eine nachhaltigere globale Entwicklung hat die UN einen Referenzrahmen geschaffen, der insbesondere durch die klare Visualisierung in Kachel- und Icon-Form sehr viel Orientierung bietet. Die Darstellung erzeugt in den Betrachter/innen das Gefühl, dass die Ziele alle zusammengehören und in die gleiche Richtung verweisen (Integration), gleichzeitig aber inhaltlich sehr disparate Entwicklungsthemen haben, die sehr unterschiedliche und teilweise widersprüchliche Maßnahmen erfordern (Differenzierung). Dieses Maß an Orientierung konnte die Debatte um eine Bildung für eine nachhaltige Entwicklung bislang nicht schaffen.

Auch wenn sich in Deutschland die Abkürzung für die englische Beschreibung der Sustainable Development Goals (SDGs) etabliert hat, scheint dies kein Hindernis in der Verbreitung zu sein. Sie geben den Akteurinnen und Akteuren im deutschen Bildungssystem vielmehr die Gelegenheit, ihr bisheriges Handeln für BNE in einen globalen Bezugsrahmen einzusortieren und über die entsprechenden Kacheln nach außen sichtbar zu machen. Vielleicht entsteht so eine große Gemeinschaft von Menschen und Institutionen, die die Zuversicht haben, mit ihren manchmal vielleicht sehr kleinen Projekten einen identifizierbaren und positionierbaren Beitrag zur deutschen Nachhaltigkeitsstrategie sowie dem globalen Zielsystem der UN leisten zu können.

Die Ziellogik der Sustainable Development Goals

Die SDGs sind ein sehr umfassendes Zielsystem für die Entwicklung eines hochkomplexen techno-sozial-ökologischen Systems, nämlich des Planeten Erde. Sie umschreiben 17 Ziele mit 169 Unterzielen. Auf Basis der Logik von Zielsystemen, bieten sich drei Ansatzpunkte für eine Auseinandersetzung mit den SDGs an:

1. Debatte über die Soll-Größen des SDG-Systems: Welcher Zustand ist für unsere Welt wünschenswert? Diese Debatte ist häufig davon geprägt, die Soll-Größen für das eigene Handeln eher leicht erreichbar zu erhalten und für andere Systeme ambitioniertere Ziele einzufordern.

2. Fundierung der Ist-Zustände der Welt: Wie sieht der entwicklungsstand der Welt empirisch aus? Wo liegen die größten Handlungsbedarfe?

3. Suche nach Gelingensbedingungen von Transformation: Wie kann der Wandel vom Ist- zum Soll-Zustand gelingen? Welche Systemhebel müssen verstellt werden, damit die Soll-Zustände erreicht werden können? Bei dieser Debatte geht es vor allem um den Wandel von Wertesystemen, welche wiederum einen Wandel in den ökonomischen und sozialen Verteilungssystemen bewirken können.

\section{Implementation und Kommunikation der Ziele im Bildungssystem}

Die Unterscheidung von Kommunikation der SDGs und Implementierung der SDGs haben wir für das deutsche Bildungssystem wie folgt vorgenommen:

1. Die SDGs zu kommunizieren, lässt sich gleichsetzen mit dem Anspruch des SDG 4.7, Bildung für nachhaltige Entwicklung allen Lernenden zugänglich zu machen. Wenn die unterschiedlichen Bildungssysteme den Lernenden die SDGs vorstellen, erklären und sie reflektieren lassen, können sie dies auf unterschiedlichen Ambitionsniveaus tun. Im konkreten Fall kann Kommunikation auch bedeuten, dass Institutionen und Akteurinnen und Akteure Projekte durchführen, deren Zweck ist, sie aktiv in die Institution hinein zu kommunizieren. Diese Art von Werbung für das Projekt ist dann eine Art informeller Bildungsprozess in einer formalen Bildungsinstitution. Eltern, die für die Kinder einen Schulgarten betreiben, werben dann bspw. mit Plakaten in der Schule und über die Schule hinaus für ihr Projekt.

2. Die SDGs zu implementieren, heißt für uns, dass die Bildungssysteme selbst versuchen, die eigene Einrichtung gemäß der SDGs umzustrukturieren. Strukturen und Abläufe, wie Bildung vermittelt wird oder entsteht, folgen den Kriterien einer nachhaltigeren Entwicklung. Beispielsweise werden die Kantinen auf mehr Bio-Ernährung umgestellt oder die Senkung des Energieverbrauchs wird angegangen. In Hochschulen ist das Thema Diversität und Heterogenität der Studierenden ein großes Thema, welches die Institutionen von innen heraus verändern. Auch diese Implementierungen können auf unterschiedlichen Ambitionsniveaus erfolgen, die im Weiteren erläutert werden.

\section{Ambitionsniveaus zur Umsetzung der SDGs}

Die Verbreitung der SDGs in Deutschland hat 2016 und 2017 einen enormen Schwung erfahren. Erstaunliche viele Akteurinnen und Akteure sowie Institutionen haben die Agenda 2030 zum Anlass genommen, dem Thema Nachhaltige Entwicklung mehr Bedeutung zu verleihen. Die Bundesregierung konnte dieser Entwicklung noch weiteres Gewicht verleihen, indem sie die 
SDGs in der Neuauflage der Deutschen Nachhaltigkeitsstrategie im Jahr 2016 integrierte und 2017 verabschiedete (Bundesregierung, 2017).

Tatsächlich ist es gar nicht einfach, einen Überblick über den Stand der Umsetzung der Ziele in Deutschland zu bekommen, da es sehr viele unterschiedliche Institutionen sind, die sich durch die Agenda 2030 und die plakativ sehr gut herausgestellten SDGs angesprochen fühlten. Aus dem eigenen Erleben heraus gab es in Wissenschaft, Politik, Wirtschaft, Kommunen und NGOs zahlreiche Veranstaltungen, die der Umsetzung der Ziele Schwung verleihen sollten. In welcher Form dieser Schwung von Kommunikationsprojekten zu neuen nachhaltigeren Strukturen in den Systemen geführt hat, lässt sich erst in einigen Jahren feststellen. Es bleibt gegenwärtig wichtig, die schwunggebenden Veranstaltungen zu identifizieren und als Good Practice zu verbreiten. Tatsächlich lassen sich auf den Homepages der Bildungsinstitutionen auf den ersten Blick nur selten eine der SDG-Kacheln finden, um das eigene Engagement einzusortieren. Auf der rhetorischen Ebene scheinen die SDGs schon stärker integriert zu werden als in der medialen Außendarstellung.

Wir bieten in dieser Studie eine Unterscheidung an, die den „Schwung“ auf drei unterschiedlichen Ambitionsniveaus untersucht. Dabei wissen wir natürlich, dass die verschiedenen Bildungssysteme in Deutschland sehr unterschiedliche Möglichkeiten haben, auf die SDGs zu reagieren, diese zu kommunizieren und im eigenen System umzusetzen.

\section{Logik der Ambitionsniveaus}

Wir gehen davon aus, dass das Thema Nachhaltigkeit - sowie auch seine Formulierung in den 17 SDGs und den 169 Unterzielen deshalb seit Jahren schon nur mühsam in alltagspraktisches Handeln von Wirtschaft, Verwaltung, NGO und Zivilgesellschaft zu integrieren ist, weil sie handfeste Dilemmata produzieren.

1. Heutige Handlungsroutinen in Wirtschaft, Verwaltung, NGOs und Zivilgesellschaft haben zu dem Zustand geführt, den wir alle als nicht mehr wünschenswert in seinen Nebenwirkungen auf Mensch und Natur betrachten. Soziale und ökologische Ressourcenquellen werden schneller ausgeschöpft, als sie sich erholen können. Gleichwohl wollen wir die Hauptwirkungen erhalten: hohe Einkommen, breite Güterversorgung, freie Mobilität. Wir wollen ansprechend gekleidet sein und als weltoffene Bürger/innen in den Urlaub reisen. Doch auch eine deutliche Steigerung der Ressourceneffizienz wird es nicht ermöglichen, dass alle Menschen auf dieser Welt unseren Lebensstand haben können, der zugleich noch wächst. Das Dilemma liegt folglich darin, dass wir nicht die Nebenwirkungen unseres wirtschaftlichen Handelns reduzieren oder reparieren können, ohne die Hauptwirkungen zu verändern. Nebenwirkungsfreier hoher Lebensstandard für alle ist auf einer Welt mit absolut knappen materiellen und immateriellen Ressourcen nicht möglich (Müller-Christ, 2007).

2. Das zweite Dilemma leitet sich aus dem ersten ab und äußert sich in Zielkonflikten zwischen den SDGs. Da diese auf die Reduzierung der Nebenwirkungen als nicht wünschenswerte Zustände reagieren, sind sie auch von den Dilemmata durchzogen, die durch die knappen materiellen und immateriellen Ressourcen der Welt entstehen. Auf der Basis heutiger Regelwerke erzeugen beispielweise verant- wortungsvollere Produktionsmuster (SDG 12) höhere Kosten, weil Nebenwirkungen reintegriert werden (externe Kosten werden internalisiert). Dadurch sinken die Gewinne, was wiederum zu geringeren Steuerzahlungen führt, aus denen hochwertige Bildung (SDG 4) finanziert werden soll.

Tatsächlich wird auch immer wieder kritisiert, dass die SDGs nicht widerspruchsfrei formuliert sind. Wir sind der Meinung, dass diese Widersprüchlichkeit der Komplexität angemessen ist und nicht aufgehoben werden kann - wohl aber bewältigt (Müller-Christ, 2014).

Die Bewältigung dieser Dilemmata stellt indes erhebliche Anforderungen an die Kompetenzen und Konfliktkultur der beteiligten Akteur/innen. Die Psychologie nennt die Fähigkeit, die Menschen brauchen, um in logischen Spannungsfeldern handlungsfähig zu bleiben, Ambiguitätstoleranz (Hartinger \& Fölling-Albers, 2005). Zudem ist es eine wichtige Fähigkeit, Konflikte und Dilemmata unterscheiden zu können: Konflikte entstehen durch unvereinbare Interessen von Akteur/inn/en und können gelöst werden, wenn die Interessen zur Situation stimmig gemacht werden. Dilemmata sind auch ohne Akteurinnen und Akteure vorhanden (die Welt hat zu wenige Ressourcen für einen hohen Lebensstandard von allen) und können nur bewältigt werden; das Handeln führt nicht zu einer Aufhebung der Spannungsfelder, sondern das Handeln muss innerhalb der Spannungsfelder stattfinden.

Diese Dilemmata können auf unterschiedlichen Ambitionsniveaus bewältigt werden. Wir gehen davon aus, dass das Ambitionsniveau der Transformationsbemühungen in Zusammenhang mit der Bereitschaft steigt, Widersprüche offen zu thematisieren und in konstruktiven Aushandlungsprozessen zu bewältigen. Wichtig ist an dieser Stelle der Hinweis, dass kein Ambitionsniveau besser oder schlechter als ein anderes ist. Auf jeder Ambitionsebene werden Menschen und Institutionen gebraucht, die die Ziele der Agenda 2030 vorantreiben. Naturgemäß werden auf dem normalen Ambitionsniveau wesentlich mehr Projekte gebraucht, weil hier mit vorhandenen Ressourcen auf bereits entwickelten Pfaden aus der Umwelt- und Nachhaltigkeitsbildung schnelle Wirkungen erzeugt werden können.

Die Idee der Ambitionsniveaus ist dem Gutachten des Wissenschaftlichen Beirats über Globale Umweltveränderungen (WBGU, 2011) zur großen Transformation entlehnt, die Widersprüchlichkeit und Dilemmata der Nachhaltigkeit sind begründet in Müller-Christ (2014).

\section{Die drei Ambitionsniveaus}

Normales Ambitionsniveau: Die 17 SDGs und ihre Unterziele lassen sich zunächst einmal einzeln umsetzen. Wir gehen davon aus, dass der erste Zugang zu dem Zielsystem für die meisten Institutionen und Akteur/innen darin liegt, die eigenen Zwecke, die bereits verfolgt werden, einem der Ziele zuzuordnen. Dies führt weitgehend zu einer Einsortierung des eigenen Handelns in das Feld der Agenda 2030. Auch Einrichtungen und Akteur/ innen, die bislang noch keines der SDGs in ihrem Handeln berücksichtigen, steigen auf dem normalen Ambitionsniveau ein, wenn sie eines der Ziele auswählen und verfolgen. Dies gilt sowohl für die Kommunikation der SDGs als auch deren Umsetzung. 
Mittleres Ambitionsniveau: Wenn Einrichtungen sich mehrere Ziele zur Kommunikation und/oder zur Umsetzung aussuchen und die Konflikte und Dilemmata zwischen diesen offen thematisieren, gehen wir von einem mittleren Ambitionsniveau aus. Gleichwohl ist es einfacher, Dilemmata zu kommunizieren als sie in der Umsetzung in der eigenen Institution auch wirklich konstruktiv zu bewältigen. In der Praxis kann man ein mittleres Ambitionsniveau daran erkennen, dass die engagierten Institutionen sowie Akteurinnen und Akteure nicht dazu neigen, ein widerspruchsfreies Zielsystem einzufordern, sondern daran, dass sie die Trade-offs der widersprüchlichen Ziele, mithin das Unvereinbare als zu bewältigende Spannung formulieren.

Hohes Ambitionsniveau: Was vielleicht nach offener Gesellschaftskritik klingt, ist in diesem Dilemmaverständnis der Versuch, die Unvereinbarkeit vieler gesellschaftlicher Ziele wie auch die Wertekonflikte moderner Gesellschaften zu thematisieren und auszuhalten. Auf diesem Ambitionsniveau geht es zunächst nicht in erster Linie darum, bestimmte SDGs zu erreichen, sondern Dialogbühnen zu schaffen, auf denen die gesellschaftlichen Akteurinnen und Akteure lernen, konstruktiv mit den Dilemmata umzugehen und die Spannungsfelder zu akzeptieren. Eine Auseinandersetzung auf dem hohen Ambitionsniveau lässt sich kommunikativ in Lehrveranstaltungen und Seminaren genauso gestalten wie in Veranstaltungen des informalen Bildungssektors, die beispielsweise moderne Konzepte nachhaltiger Wirtschaftsweisen vermitteln und diskutieren wollen. Eine Implementierung der SDGs auf hohem Ambitionsniveau wird sich im Bildungssektor dann beobachten lassen, wenn neue Bildungsanbieter moderne Bildungsprozesse auf Augenhöhe gestalten wollen, die in der Gestaltung der Bildungsprozesse die SDGs genauso berücksichtigen wie sie sie als Bildungsinhalt lehren. Wenn man die verschiedenen Ambitionsniveaus mit den unterschiedlichen Bildungsstufen in Verbindung setzt, entsteht ein Bildungsraum, in dem viele Projekte, Veranstaltungen, LehrLern-Arrangements und Konzepte ihren Platz finden können. Die zugrundeliegende Idee der Metapher des Bildungsraumes für die SDGs lautet: Je voller der Raum mit Angeboten ist, desto größer ist die Wirkung in Richtung einer nachhaltigen Entwicklung. Das Raumbild erlaubt dann auch noch einmal die Unterscheidung, dass es auf der Ebene des normalen Ambitionsniveaus sehr viele Angebote braucht, die wie ein Fundament wirken für die komplexeren Lehr-Lern-Arrangements der nächsten Stufen. Jede Stufe produziert seine eigene Wirkungsqualität und ist für die Erreichung der SDGs gleichermaßen wichtig.

\section{Die SDGs in den Bildungsstufen}

Wie nähern sich die unterschiedlichen Bildungsbereiche den Nachhaltigkeitszielen der UN? Im Folgenden soll diese Frage kurz beantwortet werden, insbesondere auch vor dem Hintergrund des Nationalen Aktionsplans (NAP) Bildung für nachhaltige Entwicklung (BNE) (Nationale Plattform Bildung für nachhaltige Entwicklung, 2017). Alle Bildungseinrichtungen haben dabei gemeinsam, dass sie die Hauptakteur/innen des Ziels SDG 4.7 (BNE in allen Bildungseinrichtungen) darstellen. Die Einschätzungen sind bewusst ohne konkrete Beispiele dargestellt, weil es eine größere Diskrepanz zwischen BNE-Engagement und Verwendung des Bezugsrahmens der SDGs in der Außendarstellung gibt.

\section{Frühkindliche Bildung}

In der frühkindlichen Bildung liegt der Fokus laut NAP BNE darauf, die Nachhaltigkeitsziele spielerisch erfahrbar zu machen. Zukunftsrelevante Fragestellungen sollen angesprochen und alltagsnah behandelt werden. Folglich liegt der Schwerpunkt auf konkreteren Nachhaltigkeitsthemen, wie den Themen Wasser (SDG 6), Ernährung (SDG 2, 3, 12), Energie (SDG 7, 12, 13) oder Gerechtigkeit (SDG 1, 5, 10). Besonders der Umgang mit unserer Umwelt scheint anschlussfähig an die gängige Ausrichtung der frühkindlichen Bildung zu sein.

Entsprechend der Zielgruppe erwarten wir in der frühkindlichen Bildung Bemühungen auf einem normalen Ambitionsniveau. Einzelne SDGs können kindgerecht aufbereitet und im alltäglichen Handeln berücksichtigt werden, beispielsweise im sozialen Umgang oder in Gartenprojekten. Dies verlangt vor allem eine nachhaltigkeitsbezogene Fortbildung und Sensibilisierung der Erziehenden und teilweise auch der Eltern. Die Umsetzung der SDGs hängt dann davon ab, ob die erwachsenen Bezugspersonen die SDGs mit Leben füllen können und beispielsweise auf ein geschlechtergerechtes Miteinander achten, was bereits aufgrund des ungleichen Geschlechterverhältnisses der Erziehungskräfte eine Herausforderung darstellen kann.

Als Hauptaufgabe der frühkindlichen Bildung sehen wir eine allgemeine Ausrichtung auf Rücksicht, Verantwortung und Umsicht an. Wichtige unterstützende Arbeitsfelder sind die Vernetzung von engagierten Erziehenden und eine nachhaltigkeitsbezogene Aus- und Fortbildung. Zudem können Einrichtungen der frühkindlichen Bildung die SDGs im Dialog mit den Eltern verwenden, einerseits zur Kommunikation des eigenen gesellschaftsverantwortlichen Handelns und andererseits auch zur Sensibilisierung der Zivilbevölkerung.

\section{Schule}

Im Schulbereich liegt der Fokus laut des Nationalen Aktionsplans BNE unter anderem darauf, „das Konzept der nachhaltigen Entwicklung als selbstverständliche Aufgabe der Bildungsverwaltung und des Bildungswesens zu verstehen" (Nationale Plattform Bildung für nachhaltige Entwicklung, 2017, S. 23) und daher auf allen Ebenen (Verwaltung, Lehrkräfte, Aus- und Fortbildung, Schule) zu integrieren.

Der Bereich Schule ist in besonderem Maße dazu geeignet, das Konzept der SDGs zu verbreiten. Durch die Vielfalt der Fächer können nahezu alle SDGs an bestehende Inhalte angeknüpft werden, sowohl in den gesellschaftswissenschaftlichen als auch in den naturwissenschaftlichen Fächern. Zudem können auf die SDGs bezogene Themen auch Inhalt im Fremdsprachenunterricht sein, beispielsweise durch Verknüpfung mit entsprechender Literatur. Mit fortschreitendem Alter der Schüler/innen können die Nachhaltigkeitsziele dabei nicht nur eindimensional, sondern auch in Bezug zueinander besprochen werden. Besonders an weiterführenden Schulen und Gymnasien bietet sich die Chance, Zielkonflikte und Widersprüche zu thematisieren, die dann in den Fächern Ethik, Politik, Philosophie, Geografie u. ä. behandelt werden können.

Der Schulbereich ist zudem offener für inhaltlichen Input von außen als beispielsweise der Hochschulbereich und lässt sich stärker durch Bildungspläne steuern. Lehrkräften bietet sich die Chance, durch die Thematisierung von Nachhaltigkeitsthemen auf die Kompetenz- und Persönlichkeitsbildung einzugehen und 
ihr Fach in globale Zusammenhänge einzubetten. Aus diesen Gründen gehen wir davon aus, dass Schulunterricht in den kommenden Jahren verstärkt ein normales Ambitionsniveau verkörpern und vielerorts auch ein mittleres Ambitionsniveau erfüllen kann.

Über den Unterricht hinaus gibt es an vielen Schulen Projekte mit Nachhaltigkeitsbezug. Schulnahe Projekte wie Schüler/innen-Firmen, Schulgärten oder Flüchtlingsprojekte bieten die Chance, einzelne SDGs in der Tiefe zu erleben und praktisch umzusetzen. Je nach Ausrichtung werden dabei auch die Zielkonflikte unterschiedlicher SDGs offenbar, sodass die Schüler/innen Handlungskompetenzen für den Umgang mit den komplexen Nachhaltigkeitsherausforderungen erwerben können. Wer bei knappem Budget und in begrenzter Zeit versucht, ökologisch, sozial und ökonomisch sinnvoll zu wirtschaften, wird merken, wie anspruchsvoll es ist, unsere globalen Herausforderungen zu bewältigen.

Das Konzept der SDGs wird unserer Recherche zufolge bislang dabei selten explizit genannt und ist im Großen und Ganzen an Schulen und in Bildungsverwaltungen unbekannt. Dennoch gibt es eine Reihe von nachhaltigkeitsnahen Zertifizierungsangeboten, wie etwa Fairtrade Schools (403 Schulen), Umweltschule in Europa - Internationale Agenda 21 Schule (800 Schulen), UNESCO Projektschulen (250 Schulen) oder EMAS Umweltschulen (131 Schulen). Etwa 1500 deutsche Schulen sind derzeit durch solche Programme zertifiziert. Als Vergleichsgröße: Laut Statistischem Bundesamt (2016) gibt es mehr als 44.000 Schulen in Deutschland, von denen mehr als $15.000 \mathrm{zu}$ den Grundschulen und etwa ebenso viele zu den weiterführenden Schulen zählen.

Insofern gibt es bereits substanzielle Bewegungen im Sinne der SDGs und gleichzeitig noch viel Handlungsbedarf, bis Nachhaltigkeit auch tatsächlich ein allgegenwärtiges Thema in Schulunterricht und -betrieb wird. Einzelnen Projektschulen mag es künftig dabei sogar gelingen, durch die nahezu allumfassende Reflektion der SDGs in Unterricht und Schulbetrieb als Ganzes ein hohes Ambitionsniveau zu verkörpern.

\section{Berufliche Bildung}

Berufliche Bildung kann einerseits an den klassischen Schulbetrieb anknüpfen, steht andererseits jedoch auch unter besonderen Anforderungen. Die Liaison mit Betrieben und die Ausrichtung auf konkrete Arbeitsfelder erzeugen einen Spannungsraum, der häufig wenig Platz für gesellschaftsrelevante Themen bietet. Im Nationalen Aktionsplan BNE liegt der Fokus daher vor allem darauf, die Zukunftsfähigkeit von beruflicher Bildung zu sichern und ökonomische Betrachtungen durch den Blick auf die gesellschaftlichen, sozialen und ökologischen Zusammenhänge im globalen Kontext zu erweitern.

Als Hauptaufgabe der Beruflichen Bildung für nachhaltige Entwicklung (BBNE) sehen wir daher eine Sensibilisierung aller Praxisakteur/innen für die gesellschaftliche Einbettung von Arbeitsprozessen. Im Idealfall werden Betriebe und Auszubildende dazu befähigt, die Folgen für Mensch und Natur in ihrem Handeln zu berücksichtigen und gleichzeitig bestehende Ansätze zukunftsorientiert weiterzuentwickeln - was einen reifen Umgang mit Zielkonflikten voraussetzt.

Diese betriebsorientierte Ausrichtung umfasst insbesondere die Nachhaltigkeitsziele für soziale Gerechtigkeit, menschen- würdige Arbeit und Gleichstellung (SDG 5, 8, 10), Energie und Umweltschutz (SDG 7, 13, 15), sowie die Weiterentwicklung von Industrie, Produktion und Konsum (SDG 9, 12). Stärker als in anderen Bildungsbereichen geht es gerade in der beruflichen Bildung darum, verantwortliches Wirtschaften zu lernen und dabei auch neue Technologien im Blick zu behalten. Der Wohlstand und Frieden Deutschlands hängt nicht zuletzt auch von der Innovationskraft der deutschen Wirtschaft ab, gepaart mit dem Mut im digitalen Zeitalter gewohnt Abläufe neu zu denken.

Dieses enorme Potenzial der beruflichen Bildung wird unserer Einschätzung nach jedoch nur von wenigen Akteur/innen gesehen und freigesetzt. Der Regelfall ist eher ein gleichbleibender Fokus auf den Mikrokosmos der jeweiligen Berufsanforderungen (s. auch die Dokumentenanalyse von Otte \& Singer-Brodowski, 2017). Zwar gibt es einige wenige Beispiele von nachhaltigkeitsbezogenen Projekten, doch haben diese eher Pilotcharakter. So gibt es bislang kaum Anzeichen für eine systematische Integration von Nachhaltigkeitsfragestellungen oder der SDGs in Unterricht und praktischer Ausbildung. Von betrieblicher Seite scheint der Fokus größtenteils darauf zu liegen, überhaupt eine genügende fachliche Qualifizierung sicher zu stellen. Insofern ist der Begriff der SDGs im beruflichen Bildungsbereich noch kaum angekommen, sodass auch das normale Ambitionsniveau nur selten verkörpert wird. Dies liegt unserer Einschätzung nach nicht an einer mangelnden Anschlussfähigkeit der SDGs an die berufliche Bildung. Vielmehr scheinen insgesamt Gedanken von Transformation und gesellschaftspolitischer Verantwortung im Sinne der UN nicht im Selbstverständnis der betrieblichen Bildung angekommen zu sein, insbesondere auch durch den wirtschaftlich durchaus verständlichen Fokus auf Fachausbildung, Effizienz und Erfolgsmaximierung.

Die Hauptherausforderung der Beruflichen Bildung für nachhaltige Entwicklung liegt unserer Einschätzung nach in den kommenden Jahren darin, BNE und die SDGs tiefer in den Curricula zu verankern und grundsätzlich alle beteiligten Stakeholder dafür zu sensibilisieren, wie wichtig die Nachhaltigkeitsziele für die Zukunftsfähigkeit der Wirtschaft sind.

\section{Hochschule}

Hochschulen stellen in Deutschland formal die Bildungseinrichtungen mit dem höchsten Komplexitätsgrad dar. Hochschulen haben dabei traditionell den Anspruch in Autonomie und Freiheit zu arbeiten. Dies bedeutet, dass sie in vielerlei Hinsicht selbstverwaltet sind und es besondere Freiheiten für Forschende und Lehrende gibt - im Gegensatz zum stärker inhaltlich regulierten Schulbetrieb. Insbesondere Professor/inn/en sollen möglichst unabhängig von äußeren Zwängen und Machtgefällen forschen und lehren können.

Gleichwohl gibt es spätestens seit den sogenannten Bologna-Reformen mehr Bewegung in Richtung Standardisierung und Qualitätssicherung. Gerade im Zuge der Exzellenz-Initiativen wird von Hochschulen erwartet, sich an internationalen Standards zu messen und eine gewisse wissenschaftliche Professionalisierung zu betreiben. Dies geht einher mit einem starken Fokus auf quantitative Indikatoren wie etwa der Höhe von eingeworbenen Drittmitteln oder der Menge an internationalen Veröffentlichungen (in möglichst hoch eingestuften wissenschaftlichen Journalen). Zudem werden durch die Akkreditie- 
rungsverfahren von Studiengängen vermehrt auch Ansprüche von außen an die Gestaltung der Lehre gestellt.

Vor diesem spannungsreichen Hintergrund stellt es eine hochkomplexe Aufgabe dar, Nachhaltigkeitsthemen und die SDGs im Hochschulbetrieb zu verankern. Das Thema der Nachhaltigkeit verlangt von hochspezialisierten Fachdisziplinen, sich für ihre gesellschaftliche Verantwortung zu öffnen und den sicheren Rahmen der eigenen Fachkultur zu verlassen. Wer sich als Wissenschaftler/in profilieren möchte, kann dies jedoch leichter innerhalb der Spielregeln des eigenen Fachs tun und dort in einem eng abgesteckten Rahmen originelle Beiträge leisten. Es gibt in der Scientific Community bislang wenig Anerkennung für das Bemühen, gesellschaftliche Transformation anzuregen und die Fachgrenzen zu überschreiten.

Gleichwohl gibt es auch zahlreiche Wissenschaftler/innen, die sich - den systemischen Dynamiken zum Trotz - Nachhaltigkeitsthemen widmen. In der Regel findet diese Auseinandersetzung innerhalb der interdisziplinären Nachhaltigkeitsszene statt; wesentlich seltener wird der Aspekt der Nachhaltigkeit in ein konkretes Fach hineingetragen und so die Fachdisziplin angereichert. Aus diesem Grund sind auch die SDGs bislang kaum in der deutschen Hochschullandschaft integriert. Der Mainstream von Forschung und Lehre hat sich noch nicht dafür geöffnet, die gesellschaftliche Einbettung des eigenen Fachs zu reflektieren.

Neben dem wissenschaftlichen Mainstream gibt es zahlreiche spannende Bewegungen, die versuchen den Freiraum von Forschung und Lehre auszuloten. So haben einige - meist kleinere - Hochschulen nachhaltige Entwicklung in ihrem Leitbild verankert. Durch Netzwerke wie HochN und einer Reihe von regionaleren Netzwerken gibt es überdies einen regen Austausch von nachhaltigkeitsinteressierten Lehrenden und Forschenden. Unserer Einschätzung nach ist den meisten Aktiven dabei bewusst, dass es bei den Nachhaltigkeitszielen nicht nur um Wissensvermittlung, sondern in besonderem Maße auch um Kompetenzentwicklung gehen muss. Da die Thematisierung der SDGs meist nicht innerhalb der Fachdisziplinen stattfindet, ist eine Großzahl der bestehenden Projekte auf mittlerem Ambitionsniveau einzustufen. Darüber hinaus gibt es erste Ansätze, das SDGs-System als Ganzes auf hohem Ambitionsniveau zu thematisieren und dabei die systemische und analytische Kompetenz der Studierenden beispielweise in neuen Studiengängen zu schulen. Über diese Entwicklungen hinaus wäre eine verstärkte Bearbeitung auf normalem Ambitionsniveau innerhalb der Fachdisziplinen wünschenswert - ähnlich wie dies teilweise im Schulbereich geschieht.

Für die Entwicklung von systemischer Kompetenz bedarf es unserer Einschätzung nach auch neuer didaktischer Methoden. In der Arbeit mit Studierenden und Organisationsvertreter/innen nutzen wir beispielsweise seit einigen Jahren die Methode der Systemaufstellung, um komplexe Zusammenhänge dreidimensional und lebendig mit Stellvertretenden zu visualisieren und so den systemischen Blick zu schulen (Müller-Christ, 2015; Müller-Christ, 2016). Weitere spannende Ansätze liegen unserer Einschätzung nach darin, weitere Transformationstheorien wie Theorie U (Scharmer, 2007), integrale Theorie (Wilber, 2007) oder Spiral Dynamics (Beck \& Cowan, 2007) in Seminaren zu diskutieren und methodisch lebendig werden zu lassen, um die Studierenden in ihrer persönlichen Kompetenz im Umgang mit Komplexität zu stärken. Diese systemische Kompetenz führt in der Folge auch dazu, dass die SDGs in ihren dynamischen Wechselbeziehungen sowie in ihrer Einbettung in eine komplexe Welt auf einem höheren Ambitionsniveau reflektiert werden können. Derlei methodische und pädagogische Experimente sind jedoch im Hochschulwesen noch Pionierprojekte und daher auch noch nicht systematisch mit den SDGs verknüpft.

Insgesamt bieten die SDGs die Chance, dass einzelne Ziele wesentlich anschlussfähiger an bestehende Inhalte sind als der schwerer zu greifende allgemeine Begriff der Nachhaltigkeit. Dabei sind unserer Einschätzung nach noch riesige Potenziale des Hochschulbereichs ungenutzt. Der Hochschulbereich ist dabei prinzipiell offen dafür, alle SDGs zu berücksichtigen. Was die Umsetzung der SDGs im eigenen Betrieb angeht, stehen darüber hinaus die Themen Wasser (SDG 6), Energie (SDG 7), gesunde und nebenwirkungsarme Ernährung (SDG 3 und 12) und Geschlechtergerechtigkeit (SDG 5) im Vordergrund.

\section{Non-formales und informelles Lernen}

Der Bereich des non-formalen und informellen Lernens zeichnet sich durch eine hohe Vielfalt und Beweglichkeit aus. Informelle Lernorte können abseits von formalen Settings nachhaltige Entwicklung erlebbar machen und die Teilnehmenden in ihrer persönlichen Transformationskompetenz unterstützen. Gleichzeitig bedeutet die geringe strukturelle Organisation auch, dass Lehrund Lernziele weniger stark von politischer Seite vorgegeben werden können und die Umsetzung von BNE noch stärker von engagierten Personen abhängt als in anderen Bildungsbereichen. Da BNE jedoch in allen Bildungsbereichen von „Überzeugungstäter/innen " abhängig ist, bietet die mangelnde Struktur auch die Chance, dass die Aktiven sich weniger auf Programme und systematische Unterstützung verlassen und stattdessen von sich aus handeln.

Dieses Spannungsverhältnis äußert sich unserer Einschätzung nach darin, dass es im Bereich des informellen Lernens einerseits eine Reihe von Vorzeigeprojekten gibt, die nachhaltige Entwicklung auf normalem Ambitionsniveau in der Tiefe bearbeiten - und es andererseits insgesamt nur eine geringe Integration der SDGs in informellen Lernprojekten zu geben scheint.

Im Nationalen Aktionsplan BNE liegt der Fokus im non-formalen und informellen Bildungsbereich darauf, die Problemlösungskompetenz von Jugendlichen sowie lebenslanges Lernen zu stärken. Jugendliche sollen als potenzielle Change Agents von morgen dazu befähigt werden, nachhaltige Entwicklung zu verstehen und leben zu können. Diese Ausrichtung auf persönliche Entwicklung und Kompetenz bedeutet, dass der informelle Bereich prinzipiell offen für alle 17 SDGs ist. Gleichwohl ist beispielsweise der sogenannte Lernlabor-Ansatz leichter auf Umwelt- und Sozialthemen anwendbar, wie SDG 6, 7, 13, 14 und 15 sowie SDG 1, 2 und 5.

Das Partnerschaftsziel SDG 17 ist dabei in allen Projekten eine praktische Herausforderung für den informellen Bereich. Denn die Akteur/innen des informellen Lernens sind im besonderen Maße auf eine kooperative und einladende Haltung der formalen Bildungsbereiche angewiesen und müssen stärker mit unterschiedlichsten Akteur/innen verhandeln, um Bewegung in Richtung BNE anzustoßen. Auf Prozessebene ist der Bereich des informellen Lernens somit stets ein Vorreiter der BNE-Vernetzung. 
Unserer Einschätzung nach hat das Ordnungsangebot der SDGs dabei eine größere Zugkraft als der Begriff „Bildung für nachhaltige Entwicklung“, sodass davon auszugehen ist, dass engagierte Akteur/innen zukünftig zunehmend von den SDGs Gebrauch machen werden, um ihre Transformationsbemühungen zu kommunizieren und bedeutsam zu machen. Der Bereich des informellen Lernens ist dabei sicherlich darauf angewiesen, dass die engagierten Akteur/innen und Akteure durch Förderprogramme für SDG-Projekte unterstützt werden, damit der Nachhaltigkeitsgedanke langfristig auch im Mainstream des informellen Lernens verankert werden kann.

\section{Fort- und Weiterbildung}

Der Bildungsbereich der Fort- und Weiterbildung ist - ähnlich dem Bereich des informellen Lernens - durch eine große Vielfalt geprägt. Stärker als in anderen Bildungsbereichen geht es hier auch um das Spiel aus Angebot und Nachfrage. Dies gilt besonders für die betrieblichen Fort- und Weiterbildungen. Doch auch in den stärker öffentlichen und zivilgesellschaftlichen Bereichen, wie beispielsweise den Volkshochschulen oder öffentlich geförderten Bildungsinitiativen, gibt es eine kaum überblickbare Bandbreite an Angeboten. Analog zum Bereich der non-formalen und informellen Bildung zeichnet sich der Bereich der Fortund Weiterbildung dadurch aus, dass es vor allem auf engagierte Köpfe ankommt und Curricula in der Regel nicht zentral oder regional vorgegeben werden.

In informellen Gesprächen haben wir immer wieder den Eindruck gewonnen, dass die SDGs bei vielen Engagierten angekommen sind, was sich jedoch noch nicht in der offiziellen Kommunikation der Projekte widerspiegelt. Tatsächlich lassen sich kaum Angebote finden, die anhand der SDGs beschrieben werden und auch den Homepages der Bildungsanbieter gibt es nur vereinzelt Hinweise auf die Agenda 2030. Allerdings beobachten wir, dass es derzeit eine Reihe von Initiativen gibt, die sich der Frage widmen, wie die SDGs in der Erwachsenenbildung integriert werden können - sei es nun im formalen oder non-formalen Bereich. Möglicherweise liegt es an der loseren Anbindung an öffentliche Bildungseinrichtungen, dass die SDGs erst jetzt im Bereich der Fort- und Weiterbildung anzukommen scheinen. Dabei sehen wir gerade auch im Bereich der Fort- und Weiterbildung das Potenzial, dass durch die SDGs das Sammelsurium an nachhaltigkeitsbezogenen Angeboten geordnet und klarer beschrieben werden kann.

Vorreiter könnten hier vermutlich besonders die öffentlich geförderten Bildungseinrichtungen sein, sofern die SDGs in den Nachhaltigkeitsstrategien der Länder und Kommunen berücksichtigt werden. Zudem könnten gerade die NGO-nahen Bildungsanbieter den ordnenden Rahmen der SDGs aufnehmen und ihre Angebote auf ihren Bezug zur globalen nachhaltigen Entwicklung prüfen. Dort wird sicherlich der Bezug zu Umwelt- und politischer Bildung im Vordergrund stehen (wie SDG 1, 2, 4, 5, 13). Im Bereich der betrieblichen Fortund Weiterbildung vermuten wir das größte Potenzial bei den Themen Energie (SDG 7) und Geschlechtergerechtigkeit (SDG 5). Themen, die das Kerngeschäft von Unternehmen (wie SDG 8, 9, 12) oder die Produktionsbedingungen (wie SDG 1, 3, 14, 15) betreffen, werden unserer Einschätzung nach erst in einigen Jahren eine größere Rolle spielen.

\section{Fazit und Handungsempfehlungen}

Gut zwei Jahre nach Verkündigung der UN Agenda 2030 und der Sustainable Development Goals soll die vorliegende Studie einen ersten Überblick geben, inwiefern die SDGs im deutschen Bildungssystem angekommen sind. Insgesamt ergibt sich dabei ein gemischtes Bild: Einerseits bleibt großes Potenzial noch ungenutzt und es bleibt noch eine Menge zu tun, bis die SDGs ein selbstverständliches Thema im deutschen Bildungssystem sind. Besonders in den Bereichen der beruflichen Bildung, des non-formalen und informellen Lernens, sowie im Bereich der Fort- und Weiterbildung ist noch viel Überzeugungs- und Kommunikationsarbeit nötig. Andererseits gibt es jedoch - zum Teil auch in den soeben genannten Bereichen - eine Vielzahl von Mut machenden Projekten, welche die SDGs auf anregende Weise behandeln und so unsere Gesellschaft ein Stück weit in Richtung einer nachhaltigen Entwicklung anschieben.

Bislang findet ein Großteil dieser Projekte auf normalem und mittlerem Ambitionsniveau statt. Gemessen am Bekanntheitsgrad der SDGs halten wir diese Entwicklung für stimmig. Zum jetzigen Stand kommt es vor allem darauf an, die Nachhaltigkeitsziele in Curricula einzubauen und mit den Fachdisziplinen zu verknüpfen. Dies stellt besonders in den stärker selbstverwalteten Bildungsbereichen eine große Herausforderung dar und ist sicherlich sowohl abhängig von politischer Unterstützung als auch vom individuellen Engagement beteiligter Akteurinnen und Akteure.

Vielerorts sind wir in unserer Recherche auf offene Türen gestoßen und konnten beobachten, dass es eine ganze Reihe von Initiativen gibt, relevante Akteurinnen und Akteure zusammen zu bringen, zu informieren und mit ihnen zu diskutieren, wie die SDGs im jeweiligen Bildungsbereich thematisiert werden können. Dies lässt die Schlussfolgerung zu, dass die Umsetzung der SDGs zwar noch in ihren Anfängen steckt, dass es gleichzeitig aber auch eine große Bereitschaft gibt, sich zu formieren und gemeinsam an Lösungen zu arbeiten. Damit diese Bereitschaft weitere Früchte tragen kann, sollen im Folgenden mögliche Handlungsfelder skizziert werden.

\section{Handlungsempfehlungen auf der Ebene der Bildungseinrichtungen}

- Bestehende Projekte und Themen den SDGs zuordnen: Sich einen Überblick verschaffen, Projekte in den globalen Kontext einordnen und die Chance nutzen, Aktivitäten wirksam darzustellen.

- Bestehende Inhalte durch die SDGs erweitern: Den Referenzrahmen nutzen, um bislang ausgeblendete Themen zu entdecken und zu integrieren.

- Werte- und Persönlichkeitsbildung fördern: Kognitiv und emotional Widersprüche und Spannungen aushalten und sich gekonnt in dynamischen Wertesystemen bewegen können.

- Betrieb von Bildungseinrichtungen nachhaltiger gestalten: Ökoeffizient, ressourcensensibel und sozial rücksichtsvoll.

- Regionale Arbeitskreise und nationale Netzwerke bilden, um voneinander und miteinander lernen zu können.

- Die SDGs in weitere Kreise tragen: Eltern, Betriebe und andere Stakeholder einbeziehen und so die SDGs in die Zivilgesellschaft tragen. 


\section{Handlungsempfehlungen auf politischer Ebene}

- Curricula und politische Vorgaben durch die SDGs anreichern

- Aus- und Fortbildung des Bildungspersonals zu den SDGs fördern

- Förderung und Verbreitung von Lehrmaterialien zu den SDGs

- Förderung von Nachhaltigkeitsinitiativen in Exzellenzwettbewerben der Hochschulen

- Nachhaltigkeitsberichterstattung inkl. der SDGs

- Vernetzung fördern, regional und national.

\section{Anmerkungen}

1 Der folgende Artikel ist eine gekürzte und überarbeitete Version der im Auftrag des Rats für Nachhaltige Entwicklung veröffentlichen „Studie zur Umsetzung der SDGs im deutschen Bildungssystem“ (Müller-Christ, Giesenbauer \& Tegeler, 2017).

\section{Literatur}

Arnold, M.-T., Carnap, A. \& Bormann, I. (2016). Bestandsaufnahme zur Verankerung von Bildung für nachhaltige Entwicklung in Bildungs- und Lehrplänen. Bericht im Auftrag der Stiftung Haus der kleinen Forscher. Berlin: Freie Universität Berlin.

Beck, D. E. \& Cowan, C. C. (2007). Spiral Dynamics - Leadership, Werte und Wandel: Eine Landkarte für Business und Gesellschaft im 21. Jahrhundert. Bielefeld: Kamphausen.

Bundesregierung (2017) (Hrsg.). Deutsche Nachhaltigkeitsstrategie - Neuauflage 2016. Zugriff unter https://www.bundesregierung.de/Content/DE/Infodienst/2017/ 01/2017-01-11-Nachhaltigkeitsstrategie/2017-01-10-Nachhaltigkeitsstrategie _2016.html.

EMAS (o.J.). Umweltschulen. Zugriff am 03.06.2018 http://www.emas.de Fairtrade Schools. Zugriff am 03.06.2018 https://www.fairtrade-schools.de Hartinger, A., Fölling-Albers, M., \& Mörtl-Hafizovic, D. (2005). Die Bedeutung der Ambiguitätstoleranz für das Lernen in situierten Lernbedingungen. Psychologie in Erziehung und Unterricht, 52(2), 113-126.

Müller-Christ, G. (2007). Umweltwirtschaft Innovativ: Organisatorische und soziale Innovationen und das Konzept der Industrial Ecology. In A. Marinuzzi \& M. Tiroch (Hrsg.), Umweltwirtschaft, Band 3. Research Institute for Managing Sustainability.

Müller-Christ, G. (2014). Nachhaltiges Management: Einführung in Ressourcenorientierung und widersprüchliche Managementrationalitäten. Stuttgart: utb.

Müller-Christ, G. (2015). Systemisches Visualisieren, Strukturbilder und FoL-ALab: Über die Weiterentwicklung der Aufstellungsmethode im Kontext der universitären Managementforschung und -lehre. In Praxis der Systemaufstellung, 2015(2), 25-39.

Müller-Christ, G. (2016). Wie kommt das Neue in die Welt? Systemaufstellungen als Instrument eines forschungsorientierten Lernens in der Managementlehre. In G. Weber \& C. Rosselet (Hrsg.), Organisationsaufstellungen. Grundlagen, Settings, Anwendungsfelder (S. 285-299). Heidelberg: Carl-Auer.
Müller-Christ, G., Giesenbauer, B. \& Tegeler, M. K. (2017). Studie zur Umsetzung der SDG im deutschen Bildungssystem. Bremen: Rat für Nachhaltige Entwicklung.

Nationale Plattform Bildung für nachhaltige Entwicklung (2017). Nationaler Aktionsplan Bildung für nachhaltige Entwicklung. Zugriff am 03.06.2018 http://www. bne-portal.de/de/infothek/publikationen/1891.

Otte, I. \& Singer-Brodowski, M. (2017). Verankerung von Bildung für nachhaltige Entwicklung in der dualen beruflichen Ausbildung. Zugriff am 03.06.2018 https:// www.researchgate.net/publication/318362220_Verankerung_von_Bildung_fur_ nachhaltige_Entwicklung_in_der_dualen_beruflichen_Ausbildung.

Scharmer, O. (2007). Theorie U. Von der Zukunft her führen. Heidelberg: Carl-Auer.

Statistisches Bundesamt (2016). Schulen auf einen Blick - Broschüre. Zugriff am 03.06.2018 https://www.destatis.de/DE/Publikationen/Thematisch/BildungForschung Kultur/Schulen/BroschuereSchulenBlick0110018169004.pdf.

Umweltschule in Europa - Internationale Agenda 21 Schule. Zugriff am 03.06.2018 http://www.umwelterziehung.de/projekte/umweltschule/.

UNESCO Projektschulen. Zugriff am 03.06.2018 http://www.unesco.de/bildung/ ups/netzwerk.html

United Nations (2018). SDG poster for non-UN entities. Zugriff am 03.06.2018 https://www.un.org/sustainabledevelopment/news/communications-material/.

WGBU (2011). Welt im Wandel: Gesellschaftsvertrag für eine Große Transformation. Zugriff am 03.06.2018 http://www.wbgu.de/hauptgutachten/hg-2011-transformation/.

Wilber, K. (2007). Integral Vision. A Very Short Introduction to the Revolutionary Integral Approach to Life, God, the Universe, and Everything. Colorado: Shambhala.

\section{Prof. Dr. Georg Müller-Christ}

ist Sprecher des Fachforums Hochschule, Mitglied der Nationalen Plattform zur Umsetzung des Weltaktionsprogramms Bildung für nachhaltige Entwicklung und Sprecher des Partnernetzwerks Hochschulen und Nachhaltigkeit. Im Rahmen des vom BMBF geförderten Projektes HochN, Hochschulen für eine nachhaltige Entwicklung, koordiniert er die Teilprojekte Vernetzung und Lehre.

\section{Bror Giesenbauer}

ist seit 2017 wissenschaftlicher Mitarbeiter im BMBF geförderten Projekt Hoch-N an der Universität Bremen und darin zuständig für die Vernetzung deutscher Hochschulen zum Thema Nachhaltigkeit. Derzeit promoviert er zu den Herausforderungen des Diversity Managements aus systemischer Perspektive. An der Jacobs University Bremen forschte er zuvor zu den Themen informelles Lernen, digitale Lernformate und Lernfitness im Alter.

\section{Merle Katrin Tegeler}

ist seit 2016 als wissenschaftliche Mitarbeiterin an der Universität Bremen im Bereich Nachhaltiges Management tätig. Sie absolvierte ihr Studium der Psychologie und Wirtschaftspsychologie an der Universität Bremen mit den Schwerpunkten Coaching, Beratung und nachhaltigem Management. Kernthema ihrer wissenschaftlichen Tätigkeit ist das BMBF-geförderte Projekt HochN und im Speziellen die Integration von $\mathrm{BNE}$ in der Lehre. 


\section{Teaching the Sustainable Development Goals - Geschichten des Wandels}

\begin{abstract}
Zusammenfassung
Das Lehrmaterial „Teaching the Sustainable Development Goals“ entstand 2017 im Rahmen des interkulturell konzipierten ESD Expert Net Mexico, India, South Africa and Germany. Das Konzept folgt der Idee, einen Bottom-up-Ansatz zu dem, was ESD-Multiplikatoren in ihrer täglichen Arbeit benötigen, mit einem Top-down-Ansatz zu den von den Vereinten Nationen vereinbarten Aufgaben, Konzepten und Programmen zu kombinieren. Das Kernelement der Handreichung besteht aus siebzehn Kapiteln, die entlang der SDGs angelegt sind. Jedes dieser Kapitel informiert über ein nachhaltiges Entwicklungsziel, warum dieses von der Generalversammlung der Vereinten Nationen artikuliert wurde sowie den diesem SDG zugeordneten Targets. Der Gestaltung des Lehrmaterials liegt die Überzeugung zugrunde, dass positive Erzählungen des Wandels eine ganz spezifische Motivation entfalten, indem sie zeigen, dass die Ziele erreicht werden können. Daher ist jedes der siebzehn Kapitel um eine solche Geschichte des Wandels herum angeordnet. Aus jedem der vier Mitgliedsländer werden vier solche Geschichten erzählt, während das SDG 17, die globale Partnerschaft, das ESD Expert Net selbst dokumentiert. Konkrete Ergänzungen zu Leitfragen, Lernzielen, Kompetenzen und Unterrichtsideen unterstützen das Lehren der SDGs in der täglichen Bildungsarbeit der vier Länder.
\end{abstract}

Schlüsselworte: Bildung für nachhaltige Entwicklung, Sustainable Development Goals, Agenda 2030, BNE-Kompetenzen

\footnotetext{
Abstract

The teaching guideline "Teaching the Sustainable Development Goals" was developed by the ESD Expert Net India, Mexico, South Africa and Germany in 2017. The concept follows the idea to combine the bottom up approach of what multipliers of ESD need in their daily work, with the top down approach of what UN-bodies agreed upon ESD issues, concepts and programmes. The core element of the guideline consists of seventeen chapters along the SDGs. Each of these chapters informs about one SDG, why this was articulated by the General Assembly of the United Nations and the related targets. The teaching guideline is based on the conviction that positive nar-
}

ratives of change do develop a specific motivation by proofing that the goals can be reached. Therefore each of the SDG chapters is concentrated around one of these stories of change. From each of the four member countries four different stories are told, while goal 17, the global partnership, documents the ESD Expert network itself. Concrete amendments on leading questions, learning goals, competencies and ideas to teach support the teaching of the SDGs in the daily education of the four countries.

Keywords: Education for sustainable development, Sustainable Development Goals, Agenda 2030, ESD competencies

\section{Einführung}

Mangrol, ein kleines Dorf an der Küste des nordwestlichen indischen Bundesstaates Gujarat, ist seit einigen Jahren Schauplatz eines bemerkenswerten Schauspiels. Entgegen der jahrhundertealten Tradition der Jagd auf Walhaie, kann seit einigen Jahren hier in den ersten Monaten des Jahres beobachtet werden, wie die Fischer mit ihren Booten aufs Arabische Meer hinausfahren, ihre Fischernetze zerschneiden und die darin gefangenen Walhaie in die Freiheit entlassen. Diese kommen jedes Frühjahr, um in den warmen Küstengewässern Nordindiens niederzukommen. Traditionell bejagten die Fischer die bis zu 12 Meter langen Riesen und sicherten so ihre Existenz. Was hat diesen Bruch mit der Tradition bewirkt? Die Meeresriesen verdanken die Wendung vor allem Morari Bapu, einem Hindupriester, der die Fischer davon überzeugte, die Tiere zu schützen. Gelungen war ihm dies, indem er die indische Tradition, wonach junge Frauen zur Geburt ihres Kindes in ihr Elternhaus zurückkehren, in Analogie zur Verhaltensweise der Walhaie setzte. Niemand, so Morari Bapu, würde einer der jungen Frauen etwas Böses tun, und so solle man es auch mit den Walhaien halten, wie es zudem der 2001 verabschiedete „Wildlife Protection Act of India“ forderte. Zum endgültigen und nachhaltig wirksamen Erfolg wurde die Geschichte dadurch, dass sich einer der industriellen Großkonzerne des Landes, Tata, bereit erklärte, die wirtschaftlichen Verluste der Fischer zu kompensieren, indem für jeden geret- 
teten Walhai ein entsprechender Geldbetrag an die Fischer ausgezahlt wird.

Geschichten, zumal Erfolgsgeschichten, rühren uns an und führen bei vielen von uns dazu, dass wir uns mit Akteuren identifizieren und uns motivieren, es den Protagonistinnen und Protagonisten gleich zu tun. Es sind positive Geschichten wie diese aus Gujarat, die Menschen bewegen und dazu führen können, eingefahrene Verhaltensweisen und Traditionen zu überdenken und diese gar im Sinne einer nachhaltigen Entwicklung zu ändern.

In dieser positiven Kraft und Wirkung von Geschichten liegt die konzeptionelle Grundüberlegung, ja Strategie des Lehr- und Lernmaterials „Teaching the Sustainable Goals“ (Hoffmann \& Gorana, 2017). Dabei stützt sich dieser Ansatz auf Überlegungen, die Harald Welzer in die Nachhaltigkeitsdiskussion eingebracht hat. Offenkundig, so Welzer (2013), mangele es hinsichtlich der angestrebten nachhaltigen Entwicklung im Rahmen einer großen Transformation nicht an Vorschlägen für technische Lösungen und auch nicht an verfügbaren, erprobten Technologien, wie dies bereits 2008 eindrucksvoll vom Forschungszentrum Karlsruhe in themengebundenen Technologiekompanden zu Energie, Wasser, Ressourcen, Boden, Klima, Luft, Abfall und Biodiversität dokumentiert wurde (Forschungszentrum Karlsruhe, 2008). Wenn die Entwicklung hin zu nachhaltigen Lebens- und Wirtschaftsweisen demnach nicht in Ermangelung verfügbarer Technologien ausbleibt, dann muss die Ursache dafür in unserer Kultur begründet liegen. Die schlüssige Folgerung Welzers (2013) lautet daher: Auf dem Weg hin zu einer nachhaltigen Entwicklung haben wir offensichtlich weniger ein technologisches als vielmehr ein kulturelles Problem zu überwinden. Die Bewältigung dieser Herausforderung aber kann nur gelingen, wenn Klarheit hinsichtlich der Funktionsweise von Kulturen besteht und Antworten auf die folgenden Fragen gegeben werden können: Was manifestiert eine Kultur? Wie kann eine Kultur verändert werden?

Die Soziologie hat auf der Suche nach Antworten die Kraft der Narrationen entdeckt und bietet folgenden Erklärungsansatz: Die spezifische Eigenheit von Kulturen basiert auf dem Zusammenwirken positiver Narrationen. Diese sind als kollektiv gelebte positive Erzählungen zu verstehen, mit denen (nahezu) jedes Mitglied einer Gesellschaft positive individuelle Erfahrungen in Verbindung bringen kann und die daher einem Kitt gleich gesellschaftlichen Zusammenhang fördern und darüber hinaus auch Identifikation schaffen. $\mathrm{Zu}$ den positiven Narrationen unserer, aber auch anderer westlicher Gesellschaften sind hier sicherlich Demokratie, Freizügigkeit, Individualität, Selbstverwirklichung, Wohlstand oder Konsum zu nennen. In diese kollektiven Erzählungen gehen Entwicklungen der Moderne ein, die in den zurückliegenden zwei Jahrhunderten zu massiven Erleichterungen und Verbesserungen im Leben sehr vieler Menschen führten, die aber auch die ökologischen und sozialen Herausforderungen hervorbrachten, deren Bewältigung wir uns heute stellen müssen. Vor diesem Hintergrund kann es also nicht darum gehen, bestehende Narrationen allesamt über Bord zu werfen, sondern vielmehr den bestehenden die der nachhaltigen Entwicklung hinzuzufügen (Welzer, 2013). Dies, so die Überlegungen Harald Welzers, holt Erfolgsgeschichten der Nachhaltigkeit aus einer vermeint-

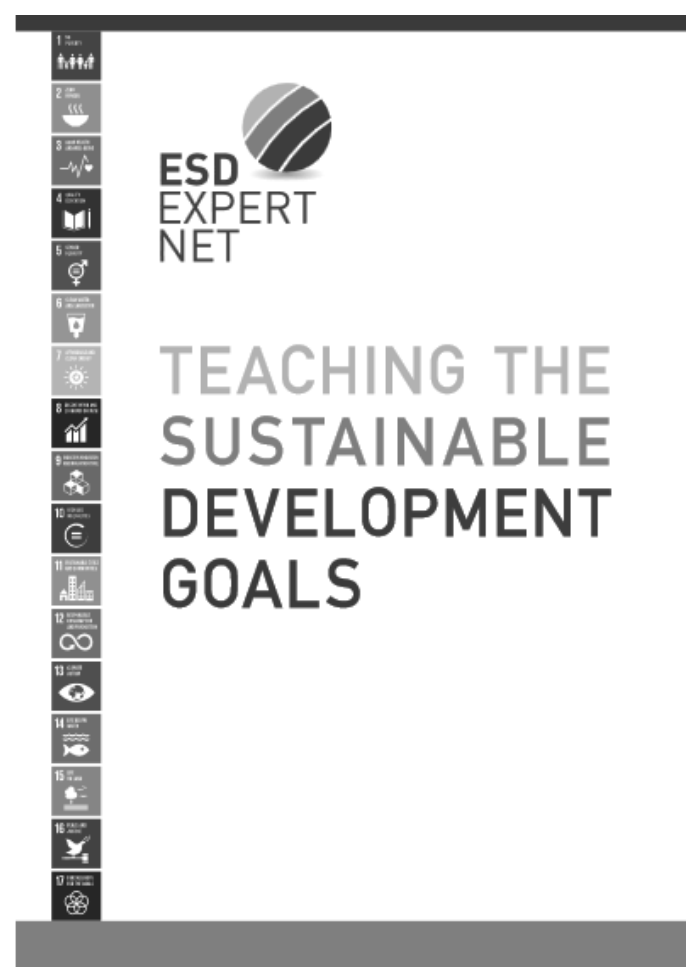

Quelle: UNESCO, 2017, S. 13

lichen Nische und rückt diese in den Wahrnehmungsbereich der Gesellschaft. In der Konsequenz dieser Überlegungen entstand die Internetseite www.futurzwei.org sowie die Ausgaben des Futurzwei-Zukunftsalmanachs (Welzer \& Rammler, 2013; Welzer et al., 2014, 2016), mittels derer hunderte Nachhaltigkeitsgeschichten einer breiten Öffentlichkeit zugängig gemacht werden und ihre Wirkung hin zur Etablierung einer weiteren Narration entfalten.

Vor diesem Hintergrund der Nachhaltigkeitsdiskussion sind die „Geschichten des Wandels“ zu verstehen, die das zentrale Element des Lehr-/Lernmaterials „Teaching the Sustainable Development Goals“ bilden. Auch lernpsychologisch erweist sich dieser Ansatz als sehr viel tragfähiger gegenüber der allzu häufig zu konstatierenden einseitigen Fokussierung auf die Problemdimensionen gegenüber der meist unbeachteten Lösungsdimension. Folge dieser in Schulbüchern wie konkretem Unterricht zu beobachtenden weit verbreiteten Praxis ist, dass die Jugendlichen mit negativen Szenarien allein gelassen werden (Hoffmann, 2018a, b). Eine Situation, aus der eher Zukunftsangst und Hoffnungslosigkeit, in den wenigsten Fällen jedoch die Motivation erwächst, der es so dringend bedarf, um die gesamtgesellschaftliche Transformation hin zu einer nachhaltig agierenden Gesellschaft zu initiieren und zu befördern. Dass der Umgang mit ungewissen Zukünften, der als zentrales Ziel der Bildung für nachhaltige Entwicklung seit Jahren diskutiert wird, auch positiv und motivierend gestaltet werden kann, unterstreicht der hier dargestellte Ansatz.

Dieser ist - wie die meisten Ansätze der Bildung für nachhaltige Entwicklung sowie des Globalen Lernens - kompetenzorientiert. Ausgehend von Kapitel 36 der „Agenda 21“, das die Diskussion über das „Wie?“ der „Förderung der Bildung, der Bewusstseinsbildung und der Aus- und Fortbildung “ eröffnete, entwickelte sich in den zurückliegenden zweieinhalb Jahrzehnten eine intensive Diskussion darüber, welches denn 
erstens die richtigen, die unbedingt benötigten Kompetenzen sind, die Lernende dazu befähigen, aktiv am gesamtgesellschaftlichen Prozess der nachhaltigen Entwicklung gestaltend teilzuhaben. Und zweitens, welche Kompetenzen Lehrende entwickelt haben müssen, um Lernende in ihrer Kompetenzentwicklung erfolgreich zu unterstützen. Beide Diskussionsstränge brachten eine Fülle interessanter Beiträge und Konzepte hervor, wobei hier lediglich auf den von der OECD vorgelegten Ansatz „Definition and Selection of Key Competencies" (DeSeCo) (OECD, 2005), den von de Haan (2009) entwickelten Ansatz der „Gestaltungskompetenz", den auf „Erkennen“, „Bewerten“ und „Handeln“ aufbauenden Ansatz des „Orientierungsrahmens“ (BMZ \& KMK, 2016), den von Rieckmann (2011) entwickelten „Konsensansatz“ sowie den synoptischen Ansatz der "Schlüsselkompetenzen“"von Wiek et al. (2011) verwiesen sei. Mit der jüngst von der UNESCO herausgegebenen Studie „Education for Sustainable Development Goals: Learning Objectives" (UNESCO 2017) scheint diese Diskussion einen breit getragenen Konsens gefunden zu haben. Danach werden folgende Kompetenzen im Kontext der Bildung für nachhaltige Entwicklung angestrebt:

- Kompetenz zum kritischen Denken

- Kompetenz zum systemischen Denken

- Kompetenz zum vorausschauenden Denken

- Normative Kompetenz

- Kompetenz zum problemlösungsorientierten Denken

- Strategische Kompetenz

- Kompetenz zur Zusammenarbeit

- Kompetenz zur Selbstwahrnehmung

Dieser Katalog an Schlüsselkompetenzen liegt dem von der UNESCO in Zusammenarbeit mit dem in Delhi ansässigen „Mahatma Gandhi Institute of Education for Peace“ (MGIEP) und Engagement Global herausgegebenen Lehrwerk „Textbook for Sustainable Development. A guide to Embedding“ (MGIEP \& UNESCO, 2017) ebenso zugrunde wie dem Lehr-/Lernmaterial „Teaching the Sustainable Development Goals“ des ESD Expert Net.

\section{Das ESD Expert Net}

Das vom Bundesministerium für wirtschaftliche Zusammenarbeit und Entwicklung (BMZ) finanzierte und von der Deutschen Gesellschaft für internationale Zusammenarbeit (GIZ) bzw. seit 2013 von Engagement Global (EG) durchgeführte Projekt "Bildung für nachhaltige Entwicklung: Deutschland, Indien, Mexiko und Südafrika" ist ein international agierendes, kooperatives Netzwerk. Ziel des Netzwerks ist es, Bildung für nachhaltige Entwicklung in den Partnerländern strukturell in allen Phasen der Lehreraus- und -fortbildung der formalen, aber auch im Rahmen der informellen Bildung zu verankern und zu stärken und so einen Beitrag zu dem im „Weltaktionsprogramm Bildung für nachhaltige Entwicklung " festgeschriebenen Motto „Vom Projekt zur Struktur“ zu leisten. Vor dem Hintergrund der UN-Dekade Bildung für nachhaltige Entwicklung (2005-14) wird erstmalig versucht, mit in ihren Regionen bedeutenden Ländern auf vier Kontinenten eine weitgehend gemeinsame und auf Augenhöhe erarbeitete Strategie zur Stärkung und strukturellen Implementierung des BNE-Ansatzes in den jeweiligen Bildungssystemen und Kulturen zu realisieren. Das übergeordnete Projektziel strebt die Stärkung der individuellen Kompetenzen und institutionellen Kapazitäten der beteiligten Organisationen an. „Innovation durch Dialog " gilt im Rahmen des Projektdesigns als zentrale Leitlinie aller Aktivitäten. Diese geht in die konzeptionellen Fragen zu BNE ebenso ein, wie in die Überlegungen, inwieweit lokales und globales Wissen in Bildungsprozessen miteinander zu verbinden sind, die Reflexionen über bestehende Veränderungspotenziale in den teilnehmenden Ländern sowie in die Stärkung der individuellen Handlungskompetenz aller am Gesamtprozess beteiligten Personen. Aus jedem der vier teilnehmenden Länder wurden maximal fünf Expertinnen und Experten benannt, die konkrete Felder des BNE-Diskurses repräsentieren. Diese sind die wissenschaftliche Beschäftigung mit dem Themenfeld Nachhaltigkeit, die erste und zweite Phase der Lehrerbildung an der Universität bzw. an einem Studienseminar, die Bildungsverwaltung sowie die Zivilgesellschaft, vertreten durch Repräsentantinnen und Repräsentanten von Nichtregierungsorganisationen. Das Projekt besteht im Wesentlichen aus folgenden Teilen:

1. Im Zentrum steht das als BNE-,, Think tank“ konzipierte Expertennetzwerk, bestehend aus je fünf Repräsentantinnen und Repräsentanten der vier teilnehmenden Länder. Dieses Gremium entwickelt die Projektideen der 3. und 4. Komponente des Gesamtprojekts und legt die weitere Zielrichtung des Gesamtprojektes fest. Im Rahmen der Projektreffen findet neben den projektinternen Workshops und Arbeitsgruppensitzungen jeweils eine eintägige öffentliche Konferenz statt, um den Austausch mit den Universitäten zu fördern, das Anliegen des Gesamtprojektes einer breiteren (Fach-)Öffentlichkeit bekannt zu machen und die Anliegen des Bildungsansatzes „Bildung für nachhaltige Entwicklung " zu befördern und dazu zu motivieren.

2. Die Internetseite des Bildungsprojektes http://esdexpert.net richtet sich an alle BNE-affinen Einrichtungen der formalen wie informellen Bildung. Neben der Hinterlegung der zentralen Dokumente der internationalen Diskussion über Bildung für nachhaltige Entwicklung sowie einer Einführung in die Zielsetzung des Projektes und Vorstellung der beteiligten Institutionen und Personen bietet die Internetseite für alle Beteiligten des Expertennetzwerkes sowie den Teilnehmerinnen und Teilnehmern des International Leadership Trainings (s. Punkt 3) einen geschlossenen Arbeitsraum.

3. Im Fortbildungsgang „International Leadership Programme“ wurden über 40 junge Berufstätige aus Bildungseinrichtungen, Nichtregierungsorganisationen (NGOs) und staatlichen Verwaltungen der Teilnehmerländer in den Bereichen Projektmanagement, Bildung für nachhaltige Entwicklung und Bildungsplanung fortgebildet. Die Teilnehmerinnen und Teilnehmer werden für einen Zeitraum von 6-8 Monaten von ihren jeweiligen Arbeitgebern freigestellt, um ein von ihnen konzipiertes Projekt im Rahmen des etwa einjährigen Fortbildungslehrganges zu vertiefen und die Feinplanungen zur Umsetzung voranzubringen. Fester Bestandteil des Ausbildungsganges ist zudem ein internationales Praktikum in einem der vier Teilnehmerländer, häufig in einer der Experten entsendenden Institutionen. Auf diese Weise soll das Lehren und Lernen sowie der Netzwerkgedanke zwischen den Teilnehmerinnen und Teilnehmern vertieft und zukünftige Kooperationsstrukturen angelegt werden. Die grundlegende Idee hinter diesem Teilprogramm ist, dass die in BNE gleichermaßen wie in Managementstrategien 
geschulten Teilnehmenden nach ihrer Rückkehr in die entsendenden Institutionen fachlich und methodisch weiter qualifiziert sind und ein konkretes Umsetzungsprojekt im Kontext ihres eigenen Arbeitsumfeldes umsetzen und so die Prozesse hin zu einer nachhaltigen Entwicklung in ihrem individuellen bzw. professionellen Umfeld weiter stärken. Informationen zu den einzelnen Projekten finden sich unter: http://esd-expert.net/what-we-do/ leadership-training. Mit dem 2017 etablierten Mentoring-Projekt findet dieser Ansatz eine Fortsetzung, indem weitere zwanzig Personen aus den vier Teilnehmerländern inhaltliche wie methodisch als BNE-Multiplikatoren fortgebildet werden.
Leadership-Training-Teilnehmer realisiert. Zudem hat sich das Netzwerk global etabliert und agiert sehr facettenreich auf der Grundlage des gemeinsamen Verständnisses von Bildung für nachhaltige Entwicklung: „Bildung für Nachhaltige Entwicklung (BNE) vermittelt Kindern, Jugendlichen und Erwachsenen, wie sie zu einer nachhaltigen Entwicklung beitragen können. Denn wir müssen lernen: Unser Handeln wirkt sich darauf aus, wie Menschen hier und in anderen Regionen der Welt heute und in Zukunft leben. Wir entscheiden mit über ihre Handlungsspielräume für eine nachhaltige Existenz. Bildung für Nachhaltige Entwicklung ist ein wichtiges Merkmal von Bildungsqualität; sie befähigt Menschen, globale Zusammenhänge zu verstehen und diese in ihrem Alltag, in ihrem Denken und Handeln zu berücksichtigen. Außerdem vermittelt sie Werte und Prinzipien, die die Basis für eine nachhaltige Entwicklung sind. Sie zeigt auf, dass Umwelt, Gesellschaft und Wirtschaft sehr komplexe Systeme sind, die eng miteinander verwoben und voneinander abhängig sind." (http://esd-expert.net/assets/BMZ_factsheet_ DE_2013_web.pdf)

Auf dieser Grundlage wurden in den zurückliegenden Jahren weitere Programme und Projekte, Arbeitsgruppen und Aktivitäten gestartet. Dazu zählt etwa das Projekt „The Turquoise Change“, das die spezifischen Bedürfnisse und Chancen von BNE im Kontext von Inseln aufgreift und zunächst auf den Andamanen und auf Sansibar umgesetzt wurde (https://www.turquoisechange.org). Dem gegenüber etabliert das Projekt „Go Global“ themenbezogen globale Lernstrukturen zwischen Bildungseinrichtungen der Partnerländer, etwa zu den Themen „Gardening“ oder "Waste“ (https://esd-expert.net/go-global-virtuellerschulaustausch.html). Die 2016 gegründete Arbeitsgruppe „Material“ baut auf der zu Projektbeginn etablierten Strategie auf, theoretische Grundsatzpapiere zu BNE zu erstellen und verfolgt darüber hinaus das Ziel, Lehrund Lernmaterialien zur Bildung für nachhaltige Entwicklung zu konzipieren und zu entwickeln. Im Rahmen dieser Arbeitsgruppe entstand jüngst in Kooperation mit „bezev“ (=

4. Im Rahmen des Teilprogrammes „Training of Trainers“ (ToT) werden vor allem Expertinnen und Experten aus dem Bereich der Lehreraus- und -fortbildung zu BNE-Multiplikatoren bzw. Beratern von Schulentwicklungsprozessen im Sinne von BNE geschult. Um dieses zu erreichen, entwickelte das Expertennetzwerk gemeinsam, also kultur- und systemübergreifend, ein Fortbildungskonzept. Die Entwicklung dieses Moduls wurde im Rahmen nationaler ToT-Projektwochen erprobt und weiterentwickelt und wird 2018 vollkommen überarbeitet in einer zweiten Auflage vorliegen.

Seit Projektbeginn wurden in den teilnehmenden Ländern hunderte Lehrerinnen und Lehrer sowie Multiplikatoren fortgebildet, Lehr- und Bildungspläne sowie Ausbildungsregularien um BNE-Aspekte erweitert und über vierzig Projekte der
Beghinderung und Entwicklungszusammenarbeit e. V.) „Cashew. Lernen mit Kernen“, ein interkulturell einsetzbare Bildungsmaterial für inklusives Lernen, sowie der hier thematisierte Band "Teaching the Sustainable Development Goals“.

\section{Konzeption}

Der Band „Teaching the Sustainable Development Goals“ richtet sich an Lehrerinnen und Lehrer, Multiplikatoren und Trainer der formalen wie der informellen Bildung vorzugsweise in den Partnerländern des ESD Expert Net, also in Indien, Mexiko, Südafrika und Deutschland. Ihre Fragen und Bedürfnisse im Kontext der alltäglichen Arbeit gingen dabei im Sinne eines Bottom-up-Ansatzes ebenso ein wie die, einem Top-down-Ansatz folgenden Bemühungen, auf der globalen Ebene entstan- 
dene und diskutierte Konzepte und Beschlüsse - etwa der UNESCO - für die Zielgruppe aufzubereiten. Folgerichtig wird im ersten Kapitel eine Standortbestimmung in der internationalen Diskussion über Bildung für nachhaltige Entwicklung und der spezifischen Kompetenzorientierung vorgenommen. Diese basiert auf der Überzeugung, dass Bildung der Schlüssel für eine positive Entwicklung im Allgemeinen und eine gelingende nachhaltige Entwicklung im Besonderen ist.

Im zweiten Kapitel wird in aller Kürze das ESD Expert Net vorgestellt und auf die verschiedenen Aktivitäten des kollaborativen Netzwerkes seit seiner Gründung 2009 verwiesen.

Die Kapitel drei „Why „Teaching the Sustainable Development Goals“?" und vier „How to use „Teaching the Sustainable Development Goals"?" dienen der konkreten Handhabung des Materials, indem die Hintergründe der Agenda 2030 im Allgemeinen und des SDG 4 bzw. des Target 4.7 in ihren Grundzügen dargelegt und die einzelnen Elemente der nachfolgenden 17 SDG-Kapitel erläutert werden. Diese bilden den Kern des Lehr-/Lernmaterials, deren immer gleich strukturierter, vierseitiger Aufbau mithilfe der Abbildung und der Nummerierungen 1 bis 12 erläutert werden soll.

1. Jedes SDG-Kapitel ist mit der offiziellen Formulierung des UN-Dokumentes „Agenda 2030“ überschrieben.

2. Jedes SDG-Kapitel zeigt das offizielle UN-Logo des jeweiligen nachhaltigen Entwicklungszieles. Das zu Beginn des Jahres 2018 neu gestaltete Logo des SDG 10 konnte nicht mehr berücksichtigt werden, so dass hier das ursprünglich von den Vereinten Nationen verwendete Logo abgebildet ist.

3. Mit dem Kapitel „Why this Goal?" werden die Leserinnen und Leser über die Entwicklungen und Dimensionen der globalen Herausforderungen informiert, die dazu führten, dass die Weltgemeinschaft das jeweilige nachhaltige Entwicklungsziel formulierte und in die Agenda 2030 aufnahm.

4. In Bild und Text berichtet eine im Kontext des jeweiligen nachhaltigen Entwicklungsziels wirksame „Geschichte des Wandels" (Story of Change) über erfolgreiche Projekte und Initiativen. Aus jedem der Projektländer Indien, Mexiko, Südafrika und Deutschland stammen jeweils vier dieser Geschichten. Die Geschichte des Wandels für das SDG 17 „Globale Partnerschaft“ erzählt die Geschichte des ESD ExpertNet als konkretes Projekt auf dieser Ebene mit der entsprechenden Zielsetzung. Mit diesen Geschichten wird einerseitsdieLückezwischen theoretischerAuseinandersetzung und alltagstauglicher Projektarbeit überwunden. Andererseits illustrieren diese Geschichten nochmals die Realitäten, die hinter der Entscheidung stehen, das jeweilige nachhaltige Entwicklungsziel zu verabschieden. Insofern dienen sie sowohl der Erläuterung für den Multiplikator als auch dem Einsatz im Unterricht als konkretes Fallbeispiel.

5. Begleitend zu der jeweiligen Geschichte des Wandels sind je nach Thema und Geschichte unterschiedlich viele SDG-Logos in unterschiedlicher Größe abgebildet: das jeweils fokussierte SDG-Thema groß, alle anderen kleiner. Mithilfe dieses graphischen Elements wird die Komplexität der vielfach interdependent miteinander verbundenen globalen Herausforderungen visualisiert und zur systemischen Auseinandersetzung mit den SDGs über die einzelnen Geschichten angeregt.
6. Der jeder Geschichte des Wandels nachfolgende Absatz „Reflect" hält eine kleine Vorschlagsliste mögliche, im Gespräch einsetzbarer Leitfragen bereit, die gleichermaßen zur Vertiefung und Reflexion der einzelnen Geschichten oder aber zur weiteren Auseinandersetzung mit dem nun eröffneten Thema genutzt werden können.

7. Unter dem Stichwort „Learning Objectives“ sind für das jeweilige SDG zentrale Lernziele gelistet, die - wenn auch in sehr viel kürzerer Form - in Übereinstimmung mit dem von der UNESCO (2017) herausgegebenen Dokument „Education for Sustainable Development Goals: Learning Objectives" stehen und somit die Verklammerung mit der internationalen Diskussion und Strategie sicherstellen.

8. Mit der Auflistung der acht Schlüsselkompetenzen, die wie oben bereits dargestellt - den Konsens der internationalen Diskussion dokumentieren, können die Nutzerinnen und Nutzer die angestrebte Kompetenzentwicklung nochmals reflektieren.

9. Unter diesem Aspekt finden sich Vorschläge für die methodisch-didaktische Unterrichtsgestaltung.

10. Hier finden sich ergänzende Informationen, die zu den Akteuren bzw. Organisationen der in den Geschichten des Wandels dargestellten Initiativen oder Projekten führen oder zur thematischen Vertiefung des SDG-Themas dienen.

11. Der Abschnitt „Ideas for your own practice“ greift maßgeblich auf Erfahrungen von WESSA (The Wildlife and Environment Society of South Africa), eine der weltweit ältesten Umweltbildungseinrichtungen zurück, die Teil des ESD Expert Net ist. Mithilfe konkreter Vorschläge wird damit die Brücke von der theoretischen und kognitiven Auseinandersetzung hin zur individuellen Handlungsoption geschlagen.

12. Unter diesem letzten Punkt sind die jedem der 17 nachhaltigen Entwicklungsziele zugeordneten „Targets“ dokumentiert. Dies eröffnet die Möglichkeit, noch weiter in die Thematik einzusteigen und auf der operativen Ebene konkrete eigene Maßnahmen zu planen und mit der übergeordneten Zielsetzung der „Agenda 2030“ zu synchronisieren.

Im Mittelpunkt dieser zu jedem SDG-Kapitel ausgearbeiteten zwölf Aspekte stehen die „Geschichten des Wandels“. Sie tragen das Potenzial, die positive Wirkung zu entfalten, die Lernende stimuliert, ihrerseits im Sinne einer nachhaltigen Entwicklung sich zu motivieren und tätig zu werden. Die nachfolgende Tabelle ermöglicht einen Überblick über die im Rahmen der Geschichten vorgestellten Initiativen und Projekte.

\section{Fazit: Erkenntnisse: Chancen und Grenzen dieses Ansatzes}

Das Lehr-/Lernmaterial „Teaching the Sustainable Goals“ ist so konzipiert, dass es in den vier Partnerländern zum Einsatz kommen kann und somit kultur- und systemübergreifend auf Augenhöhe voneinander gelernt werden kann, Kooperationen entstehen und Ideen ausgetauscht werden können. Daher sollen neben der bereits vorliegenden englischsprachigen Ausgabe weitere Ausgaben auf Spanisch und Deutsch sowie in ein oder zwei indischen Regionalsprachen - gedacht ist an Gujarati und an Maharati - zur Verfügung gestellt werden. Zugleich steht das Ziel im Raum, weitere Geschichten des Wandels aus den Part- 


\begin{tabular}{|c|c|c|c|}
\hline SDG & Initiative, Projekt & Kurzbeschreibung & Land \\
\hline $\begin{array}{l}1 \text { Armut in all ihren Formen und } \\
\text { überall beenden }\end{array}$ & $\begin{array}{l}\text { SEWA: Self Employed Womens } \\
\text { Association }\end{array}$ & $\begin{array}{l}\text { Frauenselbsthilfegruppe zur Verbesserung der } \\
\text { ökonomischen Situation und Eigenständigkeit } \\
\text { armer Frauen in Gujarat, Indien }\end{array}$ & Indien \\
\hline $\begin{array}{l}2 \text { Ernährung sichern - den Hun- } \\
\text { ger beenden, Ernährungssicher- } \\
\text { heit und eine bessere Ernährung } \\
\text { erreichen und eine nachhaltlige } \\
\text { Landwirtschaft fördern }\end{array}$ & Dabbawallas & $\begin{array}{l}\text { In Mumbai etabliertes System der Lieferung } \\
\text { privater Mittagessen an Beschäftigte mit } \\
\text { positiven Auswirkung auf soziale und ökono- } \\
\text { mische Aspekte verschiedener Bevölkerungs- } \\
\text { und Akteursgruppen }\end{array}$ & Indien \\
\hline $\begin{array}{l}3 \text { Gesundes Leben für alle - ein } \\
\text { gesundes Leben für alle Menschen } \\
\text { jeden Alters gewährleisten und ihr } \\
\text { Wohlergehen fördern }\end{array}$ & The Guardians of health & $\begin{array}{l}\text { Mit der Ausbildung von Hygiene- und Ge- } \\
\text { sundheitswächtern wird die sanitäre und } \\
\text { medizinische Situation in den teilhabenden } \\
\text { Gemeinden Mexikos verbessert. }\end{array}$ & Mexiko \\
\hline $\begin{array}{l}4 \text { Bildung für alle - inklusive, ge- } \\
\text { rechte und hochwertige Bildung } \\
\text { gewährleisten und Möglichkeiten } \\
\text { des lebenslangen Lernens für alle } \\
\text { fördern }\end{array}$ & $\begin{array}{l}\text { The dental prophylaxes container } \\
\text { in Inhambane }\end{array}$ & $\begin{array}{l}\text { Das deutsch-mosambikanische Bildungspro- } \\
\text { jekt zielt durch wechselseitige Ausbildungs- } \\
\text { phasen auf die Verbesserung prophylaktischer } \\
\text { Maßnahmen im zahnmedizinischen Bereich }\end{array}$ & Deutschland \\
\hline $\begin{array}{l}5 \text { Gleichstellung der Geschlech- } \\
\text { ter - Geschlechtergleichstellung } \\
\text { erreichen und alle Frauen und } \\
\text { Mädchen zur Selbstbestimmung } \\
\text { befähigen }\end{array}$ & $\begin{array}{l}\text { Hamburg South Africa, where } \\
\text { collaborative enterprise developed } \\
\text { new freedoms }\end{array}$ & $\begin{array}{l}\text { Durch die Entwicklung und Produktion von } \\
\text { Kunsthandwerk gelingt es den in der lokalen } \\
\text { Kooperative zusammenarbeitenden Frauen, } \\
\text { die schweren Folgen der Apartheid, der Ar- } \\
\text { beitskräftemigration der Männer sowie der } \\
\text { Aids-Problematik zumindest zu verbessern, } \\
\text { teilweise gar zu überwinden. }\end{array}$ & Südafrika \\
\hline $\begin{array}{l}6 \text { Wasser und Sanitärversorgung } \\
\text { für alle - Verfügbarkeit und } \\
\text { nachhaltige Bewirtschaftung von } \\
\text { Wasser und Sanitärversorgung für } \\
\text { alle gewährleisten }\end{array}$ & $\begin{array}{l}\text { Citizen science bio-monitoring } \\
\text { from South African MiniSASS } \\
\text { to global uptake }\end{array}$ & $\begin{array}{l}\text { Entwicklung eines Analysesets zur Bestimmung } \\
\text { der Wasserqualität und zugleich Beitrag der } \\
\text { „social science“ zum nationalem Gewässer- } \\
\text { monitoring }\end{array}$ & Südafrika \\
\hline $\begin{array}{l}7 \text { Nachhaltige und moderne } \\
\text { Energie für alle }- \text { Zugang zu } \\
\text { bezahlbarer, verlässlicher, nach- } \\
\text { haltiger und zeitgemäßer Energie } \\
\text { für alle sichern }\end{array}$ & $\begin{array}{l}\text { Renewable and independant: } \\
\text { Jühndes energy supply }\end{array}$ & $\begin{array}{l}\text { Von der übergeordneten Versorgung abgekop- } \\
\text { peltes, energieautarkes „Bio-Energiedorf“ in } \\
\text { Niedersachsen, bei dem die Dorfbewohner zu- } \\
\text { gleich Mehrheitseigner der Biogasanlagen sind }\end{array}$ & Deutschland \\
\hline $\begin{array}{l}\text { Nachhaltiges Wirtschafts- } \\
\text { wachstum und menschenwürdige } \\
\text { Arbeit für alle-dauerhaftes, brei- } \\
\text { tenwirksames und nachhaltiges } \\
\text { Wirtschaftswachstum, produktive } \\
\text { Vollbeschäftigung und menschen- } \\
\text { würdige Arbeit für alle fördern }\end{array}$ & $\begin{array}{l}\text { YES: South African Youth Em- } \\
\text { ployment Service }\end{array}$ & $\begin{array}{l}2003 \text { initiiertes Projekt zur Überwindung der } \\
\text { Arbeitslosigkeit von Frauen sowie behinder- } \\
\text { ten oder straffällig gewordenen Jugendlichen } \\
\text { durch Fortbildungsmaßnahmen und konkrete } \\
\text { Schulungen }\end{array}$ & Südafrika \\
\hline $\begin{array}{l}9 \text { Widerstandsfähige Infrastruk- } \\
\text { tur und nachhaltige Industriali- } \\
\text { sierung-eine widerstandsfähige } \\
\text { Infrastruktur aufbauen, brei- } \\
\text { tenwirksame und nachhaltige } \\
\text { Industrialisierung fördern und } \\
\text { Innovationen unterstützen }\end{array}$ & Sustainable Water-saving devises & $\begin{array}{l}\text { Entwicklung innovativer wassersparender } \\
\text { Armaturen in einem strikt am Nachhaltig- } \\
\text { keitsprinzip ausgerichteten Unternehmen }\end{array}$ & Deutschland \\
\hline $\begin{array}{l}10 \text { Ungleichheit verringern - } \\
\text { Ungleichheit in und zwischen } \\
\text { Ländern verringern }\end{array}$ & Stay or leave? & $\begin{array}{l}2007 \text { von einem Geistlichen geschaffene Ein- } \\
\text { richtung für süd- und zentralamerikanische } \\
\text { Migranten, die von Mexiko aus versuchen, die } \\
\text { USA zu erreichen }\end{array}$ & Mexiko \\
\hline
\end{tabular}




\begin{tabular}{|c|c|c|c|}
\hline $\begin{array}{l}11 \text { Nachhaltige Städte und Sied- } \\
\text { lungen - Städte und Siedlungen } \\
\text { inklusiv, sicher, widerstandsfähig } \\
\text { und nachhaltig gestalten }\end{array}$ & $\begin{array}{l}\text { Sustainability Focus: The „Green } \\
\text { City“ of Freiburg }\end{array}$ & $\begin{array}{l}\text { Das Freiburger Stadtentwicklungsprojekt } \\
\text { "Quartier Vauban“ wird als Beispiel einer } \\
\text { gelungenen nachhaltigen Stadtentwicklung } \\
\text { vorgestellt. }\end{array}$ & Deutschland \\
\hline $\begin{array}{l}12 \text { Nachhaltige Konsum- und } \\
\text { Produktionsweisen - nachhaltige } \\
\text { Konsum- und Produktionsmuster } \\
\text { sicherstellen }\end{array}$ & The green Tanguis: La Comuna & $\begin{array}{l}\text { Mit sozialen Einrichtungen kombinierte } \\
\text { ländliche Märkte, auf denen ausschließlich } \\
\text { regionale, nachhaltig produzierte Waren } \\
\text { von lokalen Produzenten und Händlern } \\
\text { angeboten werden }\end{array}$ & Mexiko \\
\hline $\begin{array}{l}13 \text { Sofortmaßnahmen ergreifen, } \\
\text { um den Klimawandel und seine } \\
\text { Auswirkungen zu bekämpfen }\end{array}$ & Leapfrogging Energy Transition & $\begin{array}{l}\text { Indiens energiepolitisches Ansinnen, die } \\
\text { Energiewende nicht im Geiste nachholender } \\
\text { Entwicklung, sondern die Fehlentwick- } \\
\text { lungen überspringend zu realisieren }\end{array}$ & Indien \\
\hline $\begin{array}{l}14 \text { Bewahrung und nachhaltige } \\
\text { Nutzung der Ozeane, Meere und } \\
\text { Meeresressourcen }\end{array}$ & $\begin{array}{l}\text { The curious case of Whali in } \\
\text { Mangrol }\end{array}$ & $\begin{array}{l}\text { Rettung der Walhaie vor der Küste Gujarats, } \\
\text { Indien durch das Zusammenwirken von lo- } \\
\text { kalem Engagement, religiöser Überzeugung, } \\
\text { Umweltschutzgesetzgebung und unterneh- } \\
\text { merischer Unterstützung }\end{array}$ & Indien \\
\hline $\begin{array}{l}15 \text { Landökosysteme schützen, } \\
\text { wiederherstellen und ihre nach- } \\
\text { haltige Nutzung fördern, Wälder } \\
\text { nachhaltig bewirtschaften, Wüs- } \\
\text { tenbildung bekämpfen, Bodende- } \\
\text { gradation beenden und umkehren } \\
\text { und dem Verlust der biologischen } \\
\text { Vielfalt ein Ende setzen }\end{array}$ & $\begin{array}{l}\text { WESSA and uMngeni-Valley - } \\
\text { caring for the earth for over } 90 \\
\text { years! Life on land the sustainable } \\
\text { way }\end{array}$ & $\begin{array}{l}\text { Jahrzehntelange Erfahrung der Verknüpfung } \\
\text { theoretischen Wissens und praktischer } \\
\text { Umsetzung in der Umweltbildung in der } \\
\text { zu gestaltenden Wirklichkeit des uMnge- } \\
\text { ni-Tales nahe Howick, Südafrika }\end{array}$ & Südafrika \\
\hline $\begin{array}{l}16 \text { Frieden, Gerechtigkeit und } \\
\text { starke Institutionen. Friedliche } \\
\text { und inklusive Gesellschaften für } \\
\text { eine nachhaltige Entwicklung för- } \\
\text { dern, allen Menschen Zugang zur } \\
\text { Justiz ermöglichen und leistungs- } \\
\text { fähige, rechenschaftspflichtige } \\
\text { und inklusive Institutionen auf } \\
\text { allen Ebenen aufbauen }\end{array}$ & $\begin{array}{l}\text { For a world, where many worlds } \\
\text { are included: Schools for peace }\end{array}$ & $\begin{array}{l}\text { Schulkonzept mit dem Ziel, auftretende } \\
\text { Konflikte und Spannungen positiv zu } \\
\text { wenden und so die soziale Kompetenz der } \\
\text { Schülerinnen und Schüler zu entwickeln } \\
\text { und zur Friedenserziehung beizutragen }\end{array}$ & Mexiko \\
\hline $\begin{array}{l}17 \text { Umsetzungsmittel stärken } \\
\text { und die globale Partnerschaft } \\
\text { für nachhaltige Entwicklung mit } \\
\text { neuem Leben füllen }\end{array}$ & ESD Expertnet & $\begin{array}{l}\text { Internationales kollaboratives Netzwerk } \\
\text { zur Bildung für nachhaltige Entwicklung } \\
\text { zwischen Indien, Mexiko, Südafrika und } \\
\text { Deutschland }\end{array}$ & $\begin{array}{l}\text { Deutschland, Indien, } \\
\text { Mexiko, Südafrika }\end{array}$ \\
\hline
\end{tabular}

Quelle: Hoffmann \& Gorana, 2017

nerländern aufzubereiten, so dass im Idealfall zu jedem nachhaltigen Entwicklungsziel eine Erzählung aus jedem Partnerland vorliegt und eingesetzt werden kann. Zugleich wird aber auch erfahrbar, dass nicht jede Idee und jedes Projekt auf die jeweils anderen Systeme und Kulturen übertragen werden kann. Dennoch steht mit dem Band „Teaching the Sustainable Development Goals“ ein zugleich kulturübergreifend nutzbares und motivierend einsetzbares Lehr-/Lernmaterial zur Verfügung Mit dem Transfer dieses Ansatzes auf andere regionale und/oder soziale Kontexte können ähnliche Sammlungen positiver Narrationen entstehen und wirken, wie es bereits auf Sansibar angestoßen wurde.

\section{Literatur}

BEZEV (Behinderung und Entwicklungszusammenarbeit e.V.) (2017) (Hrsg.). Cashew. Lernen mit Kernen. Essen: BEZEV.

Bundesministerium für wirtschaftliche Zusammenarbeit und Entwicklung und Kultusministerkonferenz (2016) (Hrsg.), Orientierungsrahmen für den Lernbereich globale Entwicklung. Bonn: Engagement Global.

Forschungszentrum Karlsruhe (2008). Roadmap Umwelttechnologien 2020. State-ofthe-Art. Karlsruhe: Forschungszentrum Karlsruhe. Zugriff am 03.06.2018 http:// www.itas.kit.edu/pub/v/2008/joua08a.pdf

de Haan, G. (2009). Bildung für nachhaltige Entwicklung - Hintergründe, Legitimationen und (neue) Kompetenzen. Zugriff am 03.06.2018 http://netzwerk-n.org/wpcontent/uploads/2017/07/de-Haan-2009-Bildung-fu\%CC\%88r-nachhaltige-Entwicklung.pdf

Hoffmann, T. (2014). Is there a specific ESD methodology? In Manual of the International ESD project Mexico, India, South Africa and Germany. http://esd-expert.net/ de/files/Concept-Paper-V_ESD-Methodology_Hoffmann.pdf

Hoffmann, T. (2016). Welche Kompetenzen für eine Bildung für nachhaltige Entwicklung brauchen Lehrende? In Lehren \& Lernen 2016(8-9), 34-38. 
Hoffmann, T. (2018a). Globale Herausforderungen. Die Zukunft, die wir wollen. Stuttgart: Ernst Klett.

Hoffmann, T. (2018b). Gerüstet für die Zukunft Aufgaben des Geographieunterrichts. In Praxis Geographie, 2018(1), 4-9.

Hoffmann, T. \& Rajeswari, G. (2017) (Hrsg.). Teaching the sustainable goals. Bonn: Engagement Global.

Hoffmann, T. \& Siege, H. (2018). What is Education for sustainable development? Unveröffentlichtes Manuskript.

Mahatma Gandhi Institute of Education for Peace (MGIEP) \& UNESCO (2017) (ed.). Textbooks for Sustainable Development. A Guide to Embedding. Delhi: Scientific and Cultural Organization Mahatma Gandhi Institute of Education for Peace and Sustainable Development.

Menon, S. \& Hoffmann, T. (2012). Out of the confusion of E-S-and D-competences. In ESD: Training of Trainers. Training Manual (S. 11-21). New Delhi 2012, 11-21. OECD: Definition and Selection of Key Competencies. Paris 2005. http://www.oecd. org/dataoecd/47/61/35070367.pdf.

Rieckmann, M. (2011). Die globale Perspektive der Bildung für eine nachhaltige Entwicklung. Eine europäisch-lateinamerikanische Studie zu Schlüsselkompetenzen für Denken und Handeln in der Weltgesellschaft. Berlin: Berliner Wissenschafts-Verlag.

UNESCO (2017). Education for Sustainable Development Goals Learning Objectives. Paris: UNESCO

Welzer, H. (2013). Selbst denken: Eine Anleitung zum Widerstand. Frankfurt/Main: S. Fischer.

Welzer, H. \& Rammler, S. (2012) (Hrsg.). FUTURZWEI-Zukunftsalmanach 2013. Frankfurt/M.: firscher.

Welzer, H., Giesecke, D. \& Tremel, L. (2014) (Hrsg.), FUTURZWEI Zukunftsalmanach 2015/16. Frankfurt/M.: Fischer Taschenbuch.

Welzer, H., Hebert, S. \& Giesecke, D. (2016) (Hrsg.). FUTURZWEI Zukunftsalmanach 2017/18. Frankfurt/M.: Fischer Taschenbuch.
Wiek, A., Withycombe, L. \& Redman, C. L. (2011). Key competencies in sustainability: a reference framework for academic program development. In Sustain Science, 6(2), 203-218.

Wissenschaftlicher Beirat der Bundesregierung Globale Umweltveränderungen (WBGU) (2011). Welt im Wandel. Gesellschaftsvertrag für eine Große Transformation. Berlin: WBGU.

Wissenschaftlicher Beirat der Bundesregierung Globale Umweltveränderungen (WBGU) (2014). Zivilisatorischer Fortschritt innerhalb planetarischer LeitplankenEin Beitrag zur SDG-Debatte. Berlin. WBGU.

\section{Dr. Thomas Hoffmann}

ist Fachleiter für Geographie am Studienseminar Karlsruhe mit den Arbeitsgebieten globale Herausforderungen, Bildung für nachhaltige Entwicklung und Geographiedidaktik sowie Lehrbeauftragter für Geographiedidaktik am Institut für Geographie und Geoökologie des Karlsruhe Institut für Technologie. Er ist Gründungsmitglied des ESD Expert Net Indien, Mexiko, Südafrika und Deutschland, das die strukturelle Implementierung von Bildung für nachhaltige Entwicklung in der Lehrerbildung der Mitgliedsländer zum Ziel hat. Im Rahmen des ESD Expert Net leitet er die Arbeitsgruppe Materialentwicklung, aus der heraus auch die hier vorgestellte Publikation entstand. In Kooperation mit der Rikkyo-Universität Tokyo erfolgt derzeit die Übertragung des Konzepts Klimawandel im regionalen Kontext auf Japan. 
Porträt

\title{
Zwei Angebote für einen besseren Materialüberblick
}

\author{
Der BNE-Kompass - \\ Außerschulische Bildungsangebote \\ für Nachhaltige Entwicklung
}

Der BNE-Kompass (www.bne-kompass.de) ist eine internetbasierte Datenbank für Bildungsangebote außerschulischer Akteure für nachhaltige Entwicklung bzw. der Sustainable Development Goals (SDGs).

Die Konzeption des BNE-Kompasses wurde im Rahmen der Nachhaltigkeitsstrategie des Landes Baden-Württemberg in einem Kooperationsprojekt des Netzwerks "Nachhaltigkeit lernen" zwischen staatlichen und zivilgesellschaftlichen Akteuren erarbeitet. Die Umsetzung der Konzeption sowie der kontinuierliche qualitative und quantitative Ausbau des BNE-Kompasses wird durch das Entwicklungspädagogische Informationszentrum (EPIZ) Reutlingen sichergestellt und seit 2011 durch das Ministerium für Kultus, Jugend und Sport in Baden-Württemberg unterstützt.

Ziel ist die möglichst flächendeckende Erfassung aller BNE-relevanten Bildungsangebote außerschulischer Bildungs-
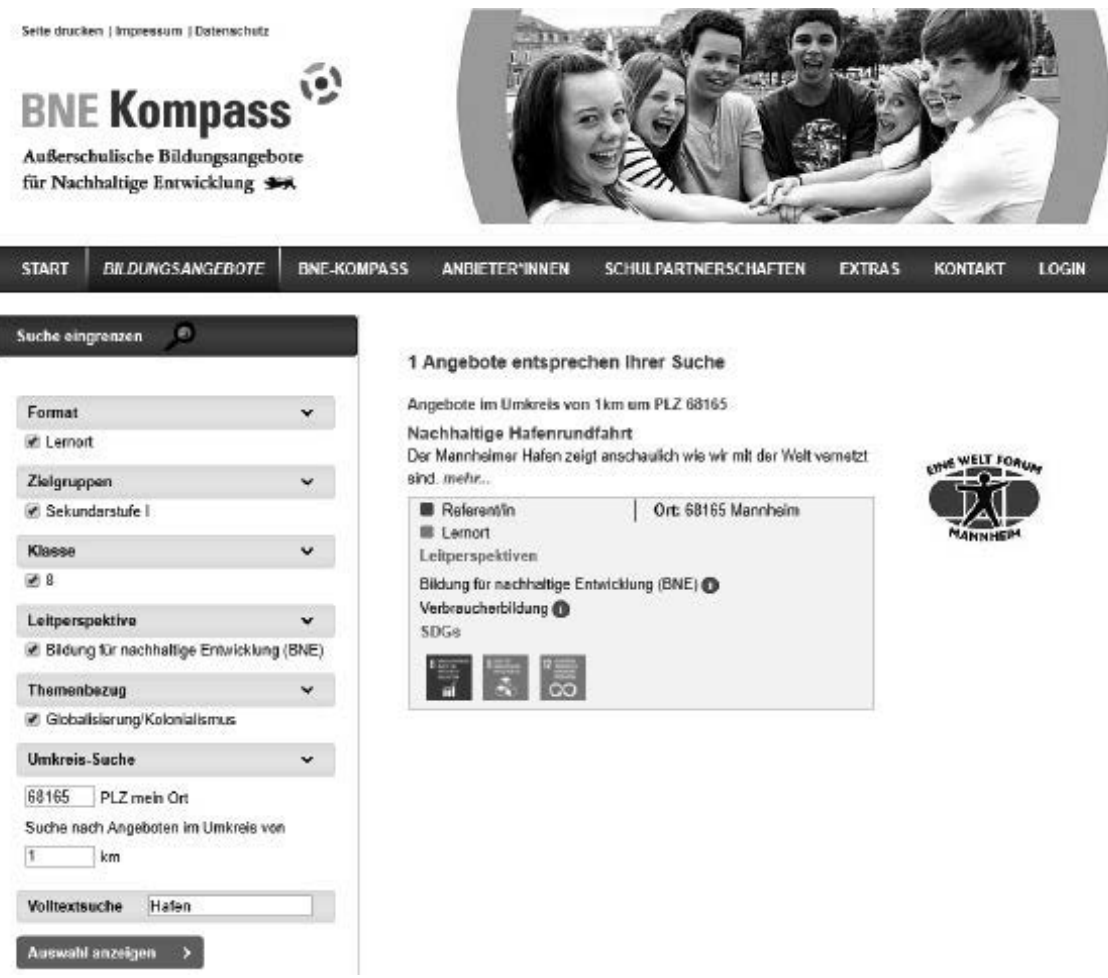

1 Angebote entsprechen threr suche

\section{Angehote im Umiseis von $1 \mathrm{~km}$ em PLZ 68165} Nachhaitige Hafenrundtahrt

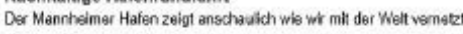
sind methe

- Roleremin - Lenor Leilperspektiven Bildung for necthatioge Entwictiung IBNE| Verberachertildung $50 G 9$

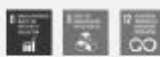

partner in Baden-Württemberg sowie die Bereitstellung dieser Angebote für die Lehrkräfte des Landes. Zur Qualitätssicherung wurde auf der Grundlage des Orientierungsrahmens für den Lernbereich Globale Entwicklung, dem Kompetenzkonzept der Gestaltungskompetenz sowie den Anforderungen des Bildungsplans 2016 für die allgemeinbildenden Schulen ein Kriterienkatalog erstellt, an dem sich die Bildungspartner mit ihren Angeboten orientieren müssen.

Diese verpflichten sich bei der Registrierung pädagogisch geschultes Personal einzusetzen und die BNE-Themenschwerpunkte bei der Gestaltung ihrer Bildungsarbeit bewusst in den Vordergrund zu stellen. In der Datenbank sind aktuell über 110 Anbieter mit mehr als 400 Angeboten registriert. Die Bildungsangebote sind nach verschiedenen Formattypen, wie z. B. Medien, Lernorte, Referent/-innen und nach Klassenstufen, Zielgruppen sowie räumlicher Entfernung selektierbar.
Aktuell wurden mit Unterstützung durch das Staatsministerium Baden-Württemberg die Auswahlkriterien an die Leitperspektiven des Bildungsplans 2016 für die allgemeinbildenden Schulen (Bildung für nachhaltige Entwicklung, Bildung für Toleranz und Akzeptanz von Vielfalt, Verbraucherbildung, Prävention und Gesundheit, etc.) und die 17 Sustainable Development Goals (SDG) angepasst und durch eine entsprechende Symbolik kenntlich gemacht.

Die Anbindung der Bildungsangebote an die Inhalte des Bil- 
dungsplans 2016 soll zum einen zu einer stärkeren Inanspruchnahme der Bildungsangebote zivilgesellschaftlicher Akteure und zum anderen zu einer stärkeren breitenwirksameren Nutzung durch die Lehrkräfte führen. Die Verlinkung der Angebote mit den Bildungsplaninhalten wird aktuell vom Landesinstitut für Schulentwicklung (LS) vorgenommen. Die Einführung des Bildungsplans 2016 hat in den letzten Monaten bereits zu einer verstärkten Nutzung durch die Lehrkräfte geführt. Diese Tendenz soll durch die aktuelle Anpassung (Leitperspektiven, SDGs) nochmals nachdrücklich unterstützt werden.

Darüber hinaus wurde das Angebot in der Kategorie „Nord-Süd“-Schulpartnerschaften um gelungene Beispiele einer gelebten SchulPARTNERSCHAFT erweitert. Des Weiteren wird der BNE-Kompass mit der neuen Internetplattform „Perspektive N“ (https://perspektive-n-bw.de) verlinkt. Diese Plattform unterstützt den Austausch von Schulen mit „nachhaltig wirtschaftenden "Unternehmen und macht sichtbar, welche Schulen und Unternehmen an einem Kontakt interessiert sind und welche Angebote bzw. Kooperationsmöglichkeiten bestehen.

Der BNE-Kompass übernimmt eine Schnittstellenfunktion zwischen formaler und non-formaler Bildung und kann als ein gelungenes Beispiel für eine konstruktive Kooperation zwischen staatlichen und zivilgesellschaftlichen Akteuren in Baden-Württemberg bezeichnet werden. Das Angebot für außerschulische Bildungspartner im Bereich der Sustainable Development Goals (SDGs) ist ein konkreter Beitrag zur Umsetzung des Ziels 4.7 der SDGs, des UNESCO-Weltaktionsprogramm „BNE“ und des Nationalen Aktionsplans BNE.

Achim Beule und Rainer Rannow kompass@epiz.de

doi.org/10.31244/zep.2018.02.06

\section{Die Lernplattform: Nachhaltige Entwicklungsziele}

\begin{abstract}
Was kann getan werden, damit die SDGs Thema in der Schule und in der Bildungsarbeit werden? Eine Antwort darauf könnte sein: Die didaktischen Materialien und Medien, die zur Verfügung stehen, bekannt machen, um die nachhaltigen Entwicklungsziele als Ausgangspunkt von Lernprozessen zufokussieren.

Acht Entwicklungsorganisationen (AWO-International, Bildung trifft Entwicklung, EWIK - Eine Welt Internet-Konferenz, Misereor, Die Sternsinger, Unicef, die Welthungerhilfe und das Welthaus Bielefeld) haben sich daher im Jahr 2015 entschlossen, eine Lernplattform aufzubauen und ins Netz zu stellen, die solche Unterstützung leistet. Unsere Lernplattform stellt bereit:

- die einschlägigen Dokumente zur Agenda 2030,

- wichtige begleitende Dokumente zu den SDGs (wie die deutsche Nachhaltigkeitsstrategie),

- Unterrichtsmaterialien verschiedener Herausgeber und Organisationen für die Sekundarstufen I und II,

- $\quad$ weitere Hinweise auf Medien, Websites, Powerpoint-Präsentationen, Material für Lehrerfortbildungen u.a.
\end{abstract}

Hier wird also ein Fundus von didaktischen Materialien und Medien zur Verfügung gestellt, der die Vielfalt der Angebote abbildet - und der laufend aktualisiert wird. Etliche Materialien wurden zudem von den Trägerorganisationen selbst erarbeitet.

Knapp zwei Jahre nach Einrichtung der Lernplattform müssen wir feststellen, dass der kurze Hype nach der Veröf- fentlichung der Agenda 2030 offensichtlich verflogen ist und mit ihm das Interesse von Lehrerinnen und Lehrern, das SDG-Weltprogramm zum Unterrichtsthema zu machen. Nur gut 300 Personen besuchen pro Monat noch unsere Website. Das ist bedauerlich, weil die Agenda 2030 ein Zukunftsprogramm formuliert, welches die Lebensgestaltung der heutigen Schülerinnen und Schüler erheblich beeinflussen wird; dies gilt im übrigen auch dann, wenn die Staatengemeinschaft die Zielsetzungen der Agenda ignorieren wird.

Wie können zivilgesellschaftliche Organisationen dazu beitragen, dass wichtige Themen im Bewusstsein von Bildungsakteuren bleiben, auch wenn die Medien längst nicht mehr davon berichten? Eine Patent-Lösung scheint es nicht zu geben, obwohl viele NROs auch heute noch auf die SDGs Bezug nehmen. Konkreter noch: Wie kann ein Website-Angebot wie unsere Lernplattform dauerhaft an die Bildungsakteure herangetragen werden? Der Sichtbarkeit im Netz sind mit unseren finanziellen Mitteln enge Grenzen gesetzt. Bezeichnenderweise fand unsere Lernplattform nicht einmal in der „Studie zur Umsetzung der SDGs im Bildungssystem“ Erwähnung, die von der Universität Bremen durchgeführt wurde.

Georg Krämer, Welthaus Bielefeld www.Lernplattform-nachhaltige-Entwicklungsziele.de doi.org/10.31244/zep.2018.02.07 


\section{BMZ-Wirkungsstudie in der Inlandsarbeit und VENRO Begleitprojekt - aktueller Stand}

Die Frage, was entwicklungspolitische Inlandsarbeit bewirken kann und wie sich diese Wirkungen feststellen lassen, wird auch bei VENRO - schon seit Jahren intensiv diskutiert. Im Rahmen des breit angelegten BMZ-Ressortforschungsvorhabens „Wirkungen und Methoden der Wirkungsbeobachtung in der entwicklungspolitischen Inlandsarbeit" wurde diesen Fragen nun systematisch nachgegangen. VENRO begleitet die seit 2016 laufende Studie durch Mitarbeit in der Steuerungsgruppe sowie durch ein Begleitprojekt, das die Beteiligung und das Mit-Lernen der Nichtregierungsorganisationen sicherstellen soll.

Die Studie geht im Wesentlichen drei übergeordneten Forschungsfragen nach:

- Welche realistischen Wirkungen lassen sich in der entwicklungspolitischen Bildungs- und Informationsarbeit erwarten und nachweisen?

- Welche Merkmale von Projekten/Maßnahmen befördern die Wirkung?

- Wie lassen sich Wirkungen mit angemessenem Aufwand erfassen?

Untersucht werden die Forschungsfragen anhand von acht Projekten, die von der Steuerungsgruppe in Zusammenarbeit mit den Mitgliedern des Konsortiums, welches das Forschungsvorhaben durchführt, ausgewählt wurden. Neben Aspekten wie unterschiedlichen Zielgruppen sowie der Verortung im schulischen und/oder außerschulischen Kontext, wurden die Projekte so ausgewählt, dass sie bestimmte „Maßnahmentypen“" (Kurzveranstaltungen/Projekttage, Multiplikator/inn/ en-Schulungen, schulbezogene Kampagnen sowie die Erstellung und Nutzung von Materialien) abdecken. Die Studie, deren Ergebnisse im Sommer 2018 veröffentlicht werden, wird empirisch unterfütterte Wirkungsmodelle für die verschiedenen Maßnahmentypen vorschlagen und beleuchten, welche Rahmenbedingungen eine wirksame Inlandsarbeit befördern können. Weiterhin sollen geeignete Instrumente zur Erfassung der Wirkungen identifiziert werden. Die Ergebnisse der Studie werden Input für weitere zivilgesellschaftliche Reflexion und Diskussionen liefern.

Anlässlich der Veröffentlichung der Studienergebnisse veranstaltet VENRO deshalb am 11. September 2018 die Konferenz „Einfach wirkungsvoll? - Erkenntnisse und Methoden der Wirkungsorientierung in der entwicklungspolitischen Inlandsarbeit" in Berlin. Auf der Konferenz sollen zum einen die Ergebnisse des Forschungsvorhabens der interessierten Öffentlichkeit präsentiert werden. Zum anderen stehen die Reflexion der Ergebnisse und die Frage, was diese für die Praxis bedeuten, im Fokus.

Flankierend zur Studie wurden von VENRO zudem Praxisworkshops angeboten, bei denen die Teilnehmenden einen Einblick in Methoden der Wirkungserfassung erhielten und diese an Praxisbeispielen diskutieren und erproben konnten. Aus diesen Workshops ist die niedrigschwellige Handreichung „Wirkungen beobachten - aber wie? Anregungen für die entwicklungspolitische Inlandsarbeit“ entstanden (online verfügbar). Die Workshops haben zudem gezeigt, dass ein Weiterlernen und Nutzbarmachen der Ergebnisse über die Studie hinaus auf großes Interesse bei NRO stößt. Weitere Workshops und Webinare sind geplant. Mehr Informationen unter: www.venro.org.

\footnotetext{
Katharina Stablecker, VENRO

k.stahlecker@venro.org doi.org/10.31244/zep.2018.02.08
} 
Faire Schule meets SDG-Labor

Die Faire Schule ist ein Schulentwicklungsprogramm, das Schulen dabei unterstützt, die Schule als Institution im Sinne des „whole school approach“ zu verändern. Das Programm zeigt auf, wie Schulen Verantwortung für eine nachhaltige Entwicklung in ihrem konkreten Handlungsumfeld übernehmen können. Schulen, die in den Bereichen demokratische Schulkultur, ökologische Verantwortung und Globales Lernen aktiv sind und ihre Aktivitäten strukturell verankern, bekommen für zwei Jahre eine Auszeichnung. Aktuell tragen fünf Berliner Schulen den Titel „Faire Schule“. Das Programm wird auch in Brandenburg durch das Diakonische Werk Teltow-Fläming und in Hamburg durch die Fachpromotorin umgesetzt. In Brandenburg gibt es vier Faire Schulen und im Herbst 2017 wurde die Stadtteilschule Bahrenfeld als erste Faire Schule Hamburgs ausgezeichnet.

Das Besondere an dem Programm ist, dass die Schulen eine enge und individuelle Beratung und Begleitung auf dem Weg zur Auszeichnung erfahren. Sie bestimmen selbst, wo sie ihre Schwerpunkte legen und welche Aktivitäten sie umsetzen möchten. Die Beratung und Begleitung unterstützt, bestärkt und regt zur Reflexion an, denn eine Schule ist immer dann gut, wenn sie bereit ist, sich weiter zu entwickeln. Wichtig ist uns dabei, dass Schulen mit der Auszeichnung die vielen tollen Aktivitäten engagierter Lehrkräfte, die bereits vorhanden sind, bündeln, sichtbarmachen und mit einem „Dach“versehen können. Durch die enge Beratung und Begleitung der Schulen finden viele kleine und große Veränderungen statt: So hat zum Beispiel die Evangelische Schule Köpenick ein Wahlpflichtfach „Nachhaltig Wirtschaften " eingerichtet, in dem Schüler/-innen sich zunächst theoretisch mit unserer globalisierten Wirtschaft und deren ökologischen und sozialen Folgen auseinandersetzen. Im zweiten Teil des Kurses arbeiten sie dann in ihrem selbst gestalteten Kiosk und bieten öko-faire Snacks und Getränke aus eigener Produktion an. An der Carlo-Schmid-Oberschule gibt es seit Neuestem das Wahlpflichtfach „Welt im Wandel“, in dem Schüler/-innen der 8. Klasse zu Themen rund um Globalisierung und Nachhaltigkeit arbeiten. Allen Fairen Schulen ist gemein, dass Schüler/-innen auf vielfältige Art und Weise Gelegenheit haben, sich in ihrer Schule zu engagieren und einzubringen. Häufig übernehmen sie Verantwortung für die jüngeren Schüler/-innen etwa durch Lernpatenschaften oder ehrenamtliche Nachhilfe. Was uns ebenfalls freut: Alle Fairen Schulen bemühen sich stark um die Integration von geflüchteten Kindern und Jugendlichen in die Schulgemeinschaft. Wir sehen also, dass das Programm und die enge Zusammenarbeit mit den Schulen auf vielfältige Weise wirken!

An Schulen, die bereits ausgezeichnet sind, führen wir SDG-Labore durch, d.h. drei- oder viertägige Projekttage, die eines der Sustainable Development Goals vertieft in den Blick nehmen. Dabei können die Schulen zwischen SDG 8 (nachhaltiges Wirtschaftswachstum), SDG 10 (Reduzierung der Ungleichheit) und SDG 12 (nachhaltige Produktion und Konsum) wählen. Neben einer Einführung in die SDGs und einem Fokus auf das ausgewählte Nachhaltigkeitsziel, entwickeln die Schüler/-innen ein kleines Forschungsprojekt und erforschen in ihrer Schulumgebung, welche Relevanz das jeweilige SDG für ihr Umfeld hat. Zu SDG 10 wurden zum Beispiel Mietpreise in verschiedenen Berliner Bezirken untersucht oder Modelle von Bücher-Tausch-Schränken entwickelt. Die Konzepte eignen sich zum Einsatz ab der 9. Klasse. Das Feedback der Schüler/-innen ist durchweg positiv. Für viele ist die intensive Auseinandersetzung mit Armut und Reichtum eine neue Erfahrung. Viele haben ihre Einschätzung über die Ursachen verändert, einige waren am Ende der Projekttage der Meinung, dass reiche Menschen mehr zur Verantwortung gezogen werden sollten, um die Ungleichheit in Berlin und auch global zu verringern. Ganz besonders haben die Schüler/-innen die Möglichkeit geschätzt, ein Forschungsprojekt zu einer Fragestellung durchzuführen, die sie selbst entwickelt haben.

Das Konzept des SDG-Labors zur Reduzierung der Ungleichheit wird für die Klassenstufen 9 und 12 ab Sommer 2018 auf der EPIZ- und EWIK-Website zum Download zur Verfügung stehen.

Nicole Humpert, EPIZ e.V. Zentrum für Globales Lernen, Berlin. humpert@epiz-berlin.de doi.org/10.31244/zep.2018.02.09 


\section{Hochwertige Bildung für Alle - Die Globale Bildungskampagne 2018 und die Bildungssituation in Albanien}

Das vierte Ziel der Agenda 2030 (Sustainable Development Goal (SDG) nennt hochwertige Bildung und die Möglichkeit lebenslangen Lernens für Alle als Voraussetzung für eine nachhaltige Entwicklung weltweit. Damit schlägt es eine Brücke zwischen den Ländern des Südens und denen des Nordens, da sich die Frage, wie qualitätsvolle Bildung finanziert und der Zugang aller dazu gewährleistet werden kann, für alle Länder stellt. Entsprechend vielfältig sind die Handlungsmöglichkeiten und -erfordernisse, die sich aus der Agenda 2030 ableiten und sich zum Beispiel in der entwicklungspolitischen Bildungsarbeit und der Finanzierung von Hochschulbildung niederschlagen.

Die Globale Bildungskampagne setzt sich 2018 unter dem Motto „Weltklasse! Bildung ist mehr wert“ für eine Erhöhung der bundesdeutschen Ausgaben für Bildung in der entwicklungspolitischen Zusammenarbeit ein. Schüler/-innen befassen sich anhand des Kampagnenmaterials mit dem Menschenrecht auf Bildung und dem Schwerpunkt Bildungsfinanzierung. Ziel der Kampagne ist es, die Schüler/-innen für Themen des Globalen Lernens zu sensibilisieren und sie in ihrem demokratischen Bewusstsein zu stärken.

Die Bedeutung des SDG 4 zeigt sich aber auch an der Bildungssituation in Albanien. Zwischen 2004 und 2010 boomte dort die Gründung privater Hochschulen, die Studierende viel Geld kosteten, qualitativ aber hinter den öffentlichen Universitäten zurückblieben. Bis heute hat das Land mit erheblichen Problemen in der Bildungsfinanzierung zu kämpfen. Ein Sur-Place Stipendium des World University Service (WUS), das vom Land Hessen finanziert wird, leistet hier einen Beitrag zu chancengerechter und hochwertiger (Hochschul-)Bildung. Es fördert junge Albaner/-innen am Ende ihres Studiums, um ihnen berufliche Zukunftsperspektiven in ihrem Heimatland zu eröffnen. Anxhela Hodaj, eine Teilnehmerin des Programms, erklärt, dass sich in den letzten Jahren schon vieles am albanischen Bildungssystem gebessert habe: „Im Vergleich zur Generation meiner Eltern gab es große Fortschritte. Es besteht mehr Chancengleichheit und wir können frei entscheiden, was wir studieren wollen, das war damals nicht möglich. " Doch es mangelt an Ausstattung und einer angemessenen Entlohnung für die Lehrenden. Wie einige Stipendiaten bemängeln, ist das Studium zu praxisfern und die Absolvent/-innen haben Probleme, einen Zugang zum Arbeitsmarkt zu finden. Im Zuge der Sur-Place-Förderung erhalten die Teilnehmenden deshalb Trainingsseminare zu Karriereplanung, Existenzgründung und Bewerbungen. In Hinblick auf die angestrebte EU-Mitgliedschaft der Westbalkanstaaten ab 2025 ist es für Albanien von großer Bedeutung, wie nachhaltig wirksame Investitionen in ein inklusives und gerechtes Bildungssystem getätigt werden können. Eine Privatisierung der Hochschulen mag den Haushalt für kurze Zeit zwar entlasten, benachteiligt aber Studierende geringverdienender Haushalte immens und fördert Möglichkeiten der Korruption.

Auch in Deutschland ist bei einer Einführung von Studiengebühren und zunehmender Privatisierung von Bildungsleistungen, eine Benachteiligung ökonomisch schwächerer Bevölkerungsgruppen zu befürchten. Die Hochschulen haben mit gravierenden Auswirkungen, u. a. einem massiven Rückgang von Studienbewerbern aus Nicht-EU-Staaten und Einschränkungen der Internationalität, zu rechnen. Dies zeigt das Beispiel Baden-Württembergs, wo seit dem Wintersemester 2017/18 durch Wissenschaftsministerin Theresia Bauer (Bündnis90/DIE GRÜNEN) Studiengebühren für Studierende aus Nicht-EU-Ländern eingeführt wurden. Die genannten Beispiele veranschaulichen, wie wichtig ein Einsatz für die Erreichung des SDG 4 der Agenda 2030 für die Bildungslandschaft weltweit ist.

Lea Faulhammer und Dr. Ursula Grünenwald, World University Service - Deutsches Komitee e. V., Informationsstelle Bildungsauftrag Nord-Süd infostelle3@wusgermany.de doi.org/10.31244/zep.2018.02.10

\section{GloNet -}

\section{das Netzwerk für Globales Lernen in der beruflichen Bildung}

Bereits im Jahr 2014 hat das EPIZ Berlin das bundesweite Vernetzungstreffen GloNet ins Leben gerufen, um Organisationen mit dem Fokus auf Globales Lernen für die berufliche Bildung in Austausch miteinander zu bringen. Das Ziel der Treffen ist es, uns gegenseitig über unsere Arbeit zu informieren, voneinander zu lernen und gemeinsame Diskussionen über aktuelle Entwick- lungen im Bereich der beruflichen Bildung zu führen. Wir wollen dabei gemeinsame Herausforderungen ebenso ausloten wie die Kooperationsmöglichkeiten untereinander. Deutschlandweit sind heute viele Organisationen im Bereich des Globalen Lernens aktiv. Berufliche Bildung steht dabei nur bei sehr wenigen im Fokus - obwohl über 50\% aller Schülerinnen und Schü- 
ler in Deutschland eine Ausbildung beginnen. Sie sind somit eine besonders wichtige Zielgruppe für Globales Lernen und BNE. Denn sie können nicht nur für zivilgesellschaftliches Engagement und kritischen Konsum sensibilisiert werden, sondern auch für nachhaltiges Wirtschaften in ihrem Betrieb und ihrer Branche.

Für Organisationen, bei denen Globales Lernen mit Zielgruppen der Beruflichen Bildung einen Schwerpunkt ihrer Arbeit darstellt, ist die Vernetzung miteinander eine große Chance: Die Herausforderungen, denen sie gegenüber stehen, sind häufig andere als in der allgemeinbildenden Schule. Lehrkräfte wünschen sich Anknüpfungspunkte dicht am Ausbildungsberuf und eine hohe Fachspezifik. Auch die duale Ausbildung, bei der die Schülerinnen und Schüler meist nur ein oder zwei Tage pro Woche in der Schule sind, stellt außerschulische Träger mit ihren Angeboten vor besondere Herausforderungen in der Kooperation. Das Thema ,heterogene Zielgruppen" betrifft die berufliche Bildung in besonderem Maße, da sich in derselben Klasse unter Umständen sowohl ehemalige Schulverweigerer/-innen als auch Studienabbrecher/-innen befinden können. Sich z.B. mit den eigenen berufsbiographischen Erfahrungen im globalen Kontext auseinanderzusetzen und sich über Lösungsstrategien auszutauschen, wird von allen teilnehmenden Organisationen als befruchtend wahrgenommen: „Der Mehrwert unserer Treffen ist absolut gegeben“, berichtet eine GloNet-Teilnehmerin über ihre Erfahrungen mit dem Netzwerk. „Allein dadurch, dass wir Lehrkräfte aus den beruflichen Schulen über weitere Projekte zu Nachhaltigkeitsthemen passgenau informieren können, lässt schon Synergien entstehen“.

Die Treffen, die etwa alle zehn Monate stattfinden, werden meist im EPIZ-Berlin durchgeführt. Wenn Ihre Organisation auch Globales Lernen für die berufliche Bildung anbietet und Sie Interesse haben, an den Vernetzungstreffen teilzunehmen, melden Sie sich gerne bei uns unter Tel. 030/69264-19, Mail: epiz@epiz-berlin.de. Aktuell sind unter anderem folgende Organisationen, Personen und Projekte bei GloNet vertreten:

BUKO Pharma-Kampagne: Die BUKO Pharma-Kampagne setzt sich für das Menschenrecht auf Gesundheit ein, für eine global gerechte Arzneimittelversorgung und für eine bedürfnisorientierte Forschung, die auch die Krankheiten der Armen im Blick hat. Unterrichtsmaterialien, Unterrichtsbesuche und Theateraufführungen an weiterführenden Schulen und Berufsschulen sind Teil der Bildungsarbeit.

Ensa-Programm - Schulaustausch für eine global gerechtere Welt: Das ENSA-Programm fördert den Austausch zwi- schen Schulgruppen aus Deutschland und Afrika, Asien, Lateinamerika oder Südosteuropa. Schulpartnerschaften werden sowohl finanziell, als auch mit einer pädagogisch-inhaltlichen Vor- und Nachbereitung unterstützt. Das ENSA-Programm möchte den internationalen partnerschaftlichen Austausch von Berufsschulen mit dem Globalen Süden im besonderen Maße unterstützen.

EPIZ Berlin, Arbeitsbereich Berufliche Bildung: Das EPIZ Berlin bietet berufsspezifische Bildungsmaterialien, Schulkooperationen, Veranstaltungen für Berlin-Brandenburger Schüler/-innen und bundesweit kostenlose Fortbildungen für Referent/-innen, Lehramtsanwärter/-innen und Lehrkräfte an.

EXILE Kulturkoordination e.V., KaffeeGartenRuhr: Mit dem „KaffeeGartenRuhr“ haben Promotor/-innen von EXILE und dem Eine Welt Netz mit dem Röster Alex Kunkel einen außerschulischen Lernort geschaffen der Lernangebote für die berufliche Bildung insbesondere für die Fachbereiche Ernährungs- und Versorgungsmanagement, Agrarwirtschaft, Wirtschaft und Verwaltung anbietet.

Fachpromotorin für Globales Lernen und Nachhaltigkeit in Hessen: Als Eine Welt-Promotorin berät Lydia Koblofsky hessenweit zu inhaltlichen wie auch pädagogischen Fragen des Globalen Lernens. Ein wichtiger Schwerpunkt ist dabei die Zusammenarbeit mit Berufsschulen und Volkshochschulen (Projekttage, Pädagogische Tage, Bildungsurlaub).

ICJA, Projekt GLOBE - Globales Lernen an Berufsschulen: Ehrenamtliche Rückkehrer/-innen aus Freiwilligendienstprogrammen des ICJA bieten in Berufs- und Fachoberschulen bundesweit Workshops zu Globalisierungsthemen an. Ziel ist es, die Schüler/-innen für Globalisierungsprozesse und ihre eigene berufliche sowie persönliche Rolle darin zu sensibilisieren.

World University Service, Projekt Grenzenlos - Globales Lernen in der beruflichen Bildung: Bei Grenzenlos vermitteln Studierende aus Afrika, Asien und Lateinamerika Themen des Globalen Lernens an berufsbildenden Schulen in Baden Württemberg, Brandenburg, Hessen, Rheinland-Pfalz und im Saarland im Unterricht, in Projektwochen oder an Projekttagen.

Silvia Kröhn und Dr. Julia Boger, World University Service - Deutsches Komitee e.V. boger@wusgermany.de www.wusgermany.de/de/auslaenderstudium/grenzenlos doi.org/10.31244/zep.2018.02.11 
Rezensionen

\section{Deutscher Bundestag (2017). Drucksache 18/13665. Bericht der Bundesregierung zur Bildung für nachhaltige Entwick- lung - 18. Legislaturperiode. 136 S., Köln: Bundesanzeiger Verlag GmbH. Verfügbar unter: http://dipbt.bundestag.de/ dip21/btd/18/136/1813665.pdf}

Einmal pro Legislaturperiode legt die Bundesregierung einen Bericht zur Bildung für nachhaltige Entwicklung (BNE) vor. Der aktuelle sechste Bericht vom 22.09.2017 befasst sich mit dem Stand und der Entwicklung von BNE in Deutschland zwischen Mitte 2013 und Mitte 2017. Damit umfasst er die letzten zwei Jahre der UN-Dekade BNE sowie den Beginn des UNESCO-Weltaktionsprogramms BNE. Nach Einschätzung der Autorinnen und Autoren hat Bildung für nachhaltige Entwicklung im Berichtszeitraum einen starken Bedeutungszuwachs erfahren. Diese Entwicklung soll im Bericht durch die Darstellung von BNE-bezogenen Aktivitäten auf unterschiedlichen Ebenen verdeutlicht werden. Der Bericht ist in acht Kapitel gegliedert, von denen die Kapitel 3 bis 7 die wesentliche inhaltliche Darlegung enthalten; arrondiert wird diese durch eine Vorbemerkung und Zusammenfassung (Kapitel 1 und 2) sowie durch einen Ausblick (Kapitel 8).

In Kapitel 3 werden die Rahmenbedingungen der BNE, konkret die Sustainable Development Goals, die UN-Dekade BNE und das Weltaktionsprogramm BNE, skizziert. Im vierten Kapitel werden der Ausbau und die Stärkung der strukturellen Verankerung von BNE auf internationaler sowie nationaler Ebene in den letzten vier Jahren beschrieben. In internationaler Perspektive wird hierzu auf Aktivitäten der UNESCO (UN-Dekade BNE, Weltaktionsprogramm BNE), der Kommission für nachhaltige Entwicklung (Hochrangiges Politisches Forum für Nachhaltige Entwicklung), der Europäischen Union, der Wirtschaftskommission der vereinten Nationen für Europa (UNECE) und der Regionalen Kompetenzzentren (RCE) eingegangen. Mit Blick auf die nationale Ebene wird die Umsetzung der UN-Dekade BNE mit einem in 2013 zum Thema „Mobilität“ ausgeschriebenen Kreativwettbewerb, rund 300 ausgezeichneten Maßnahmen, Kommunen und Projekten (in 2013 und 2014), „zahlreichen Publikationen [...] zur strukturellen Verankerung der BNE“ (S. 10) und der Abschlusskonferenz „UN-Dekade mimt Wirkung - 10 Jahre Bildung für nachhaltige Entwicklung in Deutschland" in 2014 beschrieben. Die Autorinnen und Autoren sehen zum Abschluss der UN-Dekade eine „maßgebliche Stärkung der BNE“ (S. 11), halten gleichzeitig fest, dass verschiedene Aspekte nicht erreicht wurden. Hierzu gehören u.a. die „durchgängige Verankerung von BNE in der formalen und non-formalen Bildung", die „umfassende Kooperation von schulischen und außerschulischen Bildungsträgern“, die „Ausgestaltung ganzheitlicher und partizipativer Methoden" sowie die „Aus- und Weiterbildung von pädagogischen Fach- und Lehrkräften sowie andern Multiplikatorinnen und Multiplikatoren" (S. 11). Dementsprechend wird auf die Zielsetzung des Nationalkomitees der UN-Dekade im Positionspapier „Zukunftsstrategie 2015+“"verwiesen, dass BNE zukünftig „vom Projekt zur
Struktur" kommen soll. Hierzu wurde im Kontext des Weltaktionsprogramm in einem „breit angelegte[n] partizipative[n] Prozess" (S. 12) ein Nationaler Aktionsplan BNE erarbeitet, der von den Autorinnen und Autoren als zentraler Meilenstein beschrieben wird: „Mit dem Nationalen Aktionsplan besteht erstmals in Deutschland eine umfassende BNE-Strategie, die von Bund, Ländern und Kommunen unterstützt wird und das Potenzial hat, BNE im deutschen Bildungssystem strukturell zu verankern" (S. 4). Ebenso wurden die Gremien der UN-Dekade für die Weiterarbeit im Weltaktionsprogramm reformiert, die Auszeichnungskriterien für BNE-Projekte weiterentwickelt und dabei auch die Jugendbeteiligung ausgebaut. Im fünften und umfangreichsten Kapitel des Berichtes werden die verschiedenen Maßnahmen der 18 Bundesressorts - vom Bundeskanzelamt über das Auswärtige Amt, das Bundesministerium für Bildung und Forschung, das Bundesministerium der Justiz und für Verbraucherschutz bis hin zum Ressort Beauftragte der Bundesregierung für Kultur und Medien - dargestellt. Mit den Maßnahmen werden sowohl unterschiedliche Bildungsbereiche wie frühkindliche, schulische- und außerschulische Kinder- und Jugendbildung sowie (berufliche) Aus- und Weiterbildung angesprochen als auch Projekte und Programme in den thematischen Schwerpunkten Energie, Ernährung, Landwirtschaft, Gesundheit, Demokratie, Teilhabe, Migration, Kultur und Inklusion benannt (z.B. die Initiativen „Nachhaltigkeit in der Waldbewirtschaftung“ und „zu gut für die Tonne“ sowie das „Bundesprogramm Ökologischer Landbau").

Der Beitrag der Länder und deren Bedeutung für die strukturelle Verankerung der BNE im Bildungssystem werden im sechsten Kapitel beschrieben. Im siebten Kapitel werden schließlich die Rolle der Kommunen und damit die lokale Ebene thematisiert.

Der Bericht bietet eine umfassende Darstellung von BNE-Aktivitäten in Deutschland in den letzten vier Jahren und ist Ausdruck einer deutlich erkennbaren Entwicklung zur Stärkung der Bildung für nachhaltige Entwicklung. Gleichzeitig bleibt an einigen Stellen offen, ob alle beschriebenen Maßnahmen einer Bildung für nachhaltige Entwicklung zuzuordnen sind. Insbesondere unter den in Kapitel 5 beschriebenen Maßnahmen der einzelnen Bundesressorts werden auch Aktivitäten zur Entwicklung von Nachhaltigkeit in der Gesellschaft genannt, die zunächst keine inhaltlichen Bildungskomponenten zum Thema Nachhaltigkeit aufweisen (z.B. der vom Bundesministerium für Arbeit und Soziales geförderte Ausbau inklusiver Bildung, S. 25). Insgesamt ist die Berichterstattung schwerpunktmäßig auf Maßnahmen und Projekte mit einem Fokus auf die ökologische Dimension der Nachhaltigkeit ausgerichtet. Nur an vergleichsweise wenigen Stellen, z.B. bei den Aktivitäten des Auswärtigen Amtes und des Bundesministeriums für wirtschaftliche Zusammenarbeit und Entwicklung, werden beispielsweise mit dem Freiwilligendienst kulturweit, dem Schulwettbewerb des Bundespräsidenten „Alle für Eine Welt - Eine Welt für Alle“ oder dem entwicklungspolitischen Schulaustauschprogramm ENSA auch die soziale und kulturelle Dimension von Nachhaltigkeit 
berücksichtigt. Ansätze und Projekte, die im Bereich Globales Lernen verortet sind, erhalten in dieser Ausrichtung wenig Aufmerksamkeit. Diese enge Fokussierung auf ökologische Inhalte wurde in der Vergangenheit bereits beobachtet, z.B. im Kontext der Umsetzung der BNE-Dekade (Deutscher Bundestag, 2000, S. 24/25). Daher gilt es auch zukünftig zu beobachten, inwiefern sich das Verständnis der BNE in Richtung einer mehrdimensionalen Betrachtung, die sozialen und kulturellen Perspektiven größere Bedeutung beimisst, entwickelt.

\section{Literatur}

Deutscher Bundestag (14. Wahlperiode) (2000). Drucksache 14/3319. Bericht der Bundesregierung zur Bildung für eine Nachhaltige Entwicklung. Zugriff am 14.02.2018, http:/www.globaleslernen.de/sites/default/files/files/link-elements/bundesregierung_20zur_20bildung_20f_c3_bcr_20nachhaltige_20entwicklung_2c_202001. pdf

Marina Wagener und Sabine Lang doi.org/10.31244/zep.2018.02.12

\section{McCloskey, S. (2014): Development Education in Policy and Practice. 300 S., Palgrave: Macmillan. 66,88€}

Auch wenn die hier zu besprechende Veröffentlichung bereits aus dem Jahr 2014 stammt, nutzen wir mit dieser Rezension die Gelegenheit, auf diesen Sammelband hinzuweisen, der aus unserer Sicht für die Debatte zur Umsetzung der SDGs ein paar interessante Impulse geben kann.

Dieser Sammelband vereint ein breites Spektrum an Autor/ inn/en, welche die aktuellen Debatten und die Weite der Themen der entwicklungspolitischen/-bezogenen Bildung wiederspiegeln. Der Herausgeber verwendet „development education “ als Oberbegriff der vielfältigen Begrifflichkeiten, die in diesem Feld - wie auch von den Autor/inn/en der Publikation - verwendet werden (darunter global citizenship education, global education oder sustainable development). Dies ist ein ansprechender Ansatz, da dies andeutet, dass die langwierigen Diskussionen zu den Begrifflichkeiten (größtenteils?) überwunden und durch einen starken Fokus auf die geteilten Inhalte ersetzt wurden. Der Klappentext postuliert hierzu: „Development education is a radical form of learning that addresses the structural causes of poverty, inequality and injustice in the global North and South. It equips learners with the skills, values, attributes and understanding needed to take effective action toward social change."

Die Veröffentlichung ist in fünf Teile gegliedert. Der erste Teil („Soft versus critical development education“) konzentriert sich auf die Diskussion von „critical literacy“ als Ziel entwicklungspolitischer Bildungsprozesse. In diesem Teil präsentiert Audrey Bryan Forschungsergebnisse einer Lehrplananalyse anhand derer Sie aufzeigt, dass im post-primaren Bereich in Irland aktuell vor allem Ansätze von „Global Citizenship“ dominieren, die auf der personenbezogenen Ebene verweilen. Andreotti betont in Kapitel 2 die Verschiebung hin zu einer „critical global citizenship education“, die sich um die Reflexion der Ursprünge von sozialer Ungerechtigkeit bemüht. Teil II („Development Education Sectors") behandelt eine Auswahl von drei Bildungsbereichen: Jugend, Lehrerausbildung und Hochschulbildung. In diesem Teil betont Fionnuala Waldron in Kapitel 7, dass Lehramtsstudierende in ihrem Studium Möglichkeiten für die kritische Reflexion von selbstverständlich geglaubten Vorstellungen zum Lehren und Lernen benötigen, zum Beispiel anhand von „troublesome spaces“. Damit verbindet die Autorin die Hoffnung der Loslösung der vordringlich verwendeten „soft“-Ansätze in der entwicklungspolitischen Bildung, die Gefahr laufen bestehende Dominanzverhältnisse zu reproduzieren statt in Frage zu stellen. Zeitgemäß - am Ende der UN-Dekade - konzentriert sich Teil III auf den Zusammenhang von entwicklungsbezogener Bildung und nachhaltiger Entwicklung. So plädiert bspw. Glenn Strachan für einen ganzheitlichen Ansatz betreffend des Klimawandels, basierend auf der Erkenntnis, dass Fragen nach Ursachen und Auswirkungen ein Problem der Gleichberechtigung und der sozialen Gerechtigkeit sind - eng verknüpft mit wirtschaftlichen Interessen, Migrationsbewegungen und anderen inhaltlich verwandten Bereichen. In Teil IV („New Development Paradigms: Lessons for Development Education“) werden globale Kontexte betrachtet, in denen entwicklungspolitische Bildungsarbeit eingebunden ist, und in Teil V („Development Education's Shifting Policy Landscape") wird die sich wandelnde bildungspolitische Situation mit Blick auf deren Wirkung auf die Bildungspraxis diskutiert. Der letzte Artikel von Stephen McCloskey bietet ein nützliches Resümee der Veröffentlichung - etwas das Sammelbänden häufig fehlt.

Das Ziel des Herausgebers "to provide the reader with a text that captures the breadth of development education" (p. 12) wird mit dieser Publikation erfüllt - insbesondere durch das Abbild der breiten Palette des Themenfelds. Die Publikation zeichnet sich dadurch aus, dass der Herausgeber (vgl. Publikationstitel) sich explizit der Herausforderung stellt, die (häufig gegenläufigen) entwicklungspolitischen und praxisbezogenen Räume in einer Publikation zu verbinden. Eine prominentere Rolle von wissenschaftlichen Forschungsergebnissen hätte nützliche „Koordinaten “für die Gesamtschau des Felds liefern können. Die fünfTeile bilden eine kohärente Struktur, die auch durch die der Autor/ inn/en anklingende angemessene kritische Haltung betreffend der Evolution der entwicklungspolitischen Bildungsarbeit überzeugt. Die Publikation ist insbesondere für Lesende gewinnbringend, die sich vertiefende Einblicke zu dem Themenspektrum sowie zu den derzeitig diskutieren Ansätzen in der entwicklungspolitischen/-bezogenen Bildung aneignen möchten.

Sarah Lange doi.org/10.31244/zep.2018.02.13 


\section{Schlaglichter}

BNE-Sommerakademie 2018 (red.): Auch 2018 veranstaltet das FORUM Umweltbildung im Auftrag des Bundesministeriums für Nachhaltigkeit und Tourismus (BMNT) in Österreich die BNE-Sommerakademie. Diese wird vom 20. bis zum 23. August in Frankenfels in Niederösterreich stattfinden. Thematisch steht die Veranstaltung unter der Überschrift „Klimafrieden - Friedensklima“. Zusammenhänge zwischen Klima und Frieden sollen diskutiert und an die Frage geknüpft werden, inwiefern individuell und im Kollektiv Verantwortung für soziale Gerechtigkeit übernommen werden kann und soll. Teilnehmen können Lehramtsstudierende, Lehrerinnen und Lehrer, Pädagogisch Tätige aus dem Kindergarten- und Hortbereich, Pädagogen und Pädagoginnen sowie Multiplikatoren aus dem außerschulischen Bereich und alle Interessierten. Weitere Informationen sind unter http://www.umweltbildung.at/veranstaltungen/forum-veranstaltungen/bne-sommerakademie. html gebündelt.

Seminar zu partizipativen Methoden in der Entwicklungszusammenarbeit (red.): Vom 4.-7. Oktober 2018 bietet Brot für die Welt ein Seminar für Fachkräfte von Diensten und Missionswerken und für Mitarbeitende in kirchlichen Hilfswerken an. Es ermöglicht einen Überblick über unterschiedliche partizipative Methoden und Möglichkeiten der Anwendungen im Alltag entwicklungspädagogischer Projekte zu gewinnen. Zentral werden die Reflexion und Erprobung ausgewählter partizipativer Instrumente aus dem Methodenpaket des „Participatory Rural Appraisal“ (PRA) sein. Die Anforderungen an einen Beratenden, der zugleich Teil eines Projekts ist, werden dabei thematisiert sowie die Chancen und Grenzen partizipativer Arbeitsweisen.

Kongress zur Freire-Pädagogik (red.): Vom 9.-11. November 2018 veranstaltet die Paulo Freire Kooperation in Zusammenarbeit mit der Fakultät für Erziehungswissenschaft der Universität Hamburg einen Kongress mit dem Titel: „Dialogisches Denken und Bildung als Praxis der Freiheit. Mit Freire den Herausforderungen einer inklusiven Gesellschaft begegnen“. Es geht zum einen um eine Bestandsaufnahme des Wirkens von Freire in verschiedenen Ländern und Arbeitsbereichen. Die Vielfalt der Anwendungsfelder soll einem breiten Fachpublikum und der öffentlichen Debatte präsentiert werden. In Zeiten der Globalisierung und Digitalisierung sind die Erfahrungen mit seiner Pädagogik zum anderen neu zu bewerten. Wie können wir die Ansätze von Freire hier und heute erfolgreich nutzen?

Auf der Tagung sind verschiedene Diskussionsforen geplant, für die wir um Beiträge bitten. Weitere Informationen, das Tagungsprogramm, die Themen der Foren und ihre ModeratorInnen sowie die Anmeldungsmodalitäten finden sie unter www.freirehamburg2018.de.
Faire Ferien 2018 (red.): Die evangelischen und katholischen Jugendverbände AEJ und BDKJ haben gemeinsam mit MISEREOR eine neue Ausgabe der „Fairen Ferien“ veröffentlicht. Die Broschüre gibt Tipps für die Gestaltung einer nachhaltigen Ferienfreizeit. Dabei steht das 2018 erschienene Aktionsmaterial ganz unter dem Motto Gutes Leben und der damit verbundenen Frage, was junge Menschen für ein gutes Leben brauchen? Verknüpft wird diese Themenstellung mit dem Konzept der nachhaltigen Entwicklung, wodurch die Jugendlichen dazu angeregt werden sollen, eigene Vorstellungen und Gewohnheiten zu hinterfragen und zu reflektieren. Das Material liefert beispielsweise zielgruppengerechte Tipps für einen plastikfreien Tag oder verschiedene aktivierende Methoden, die mit Naturmaterialien durchgeführt werden können. Die Broschüre „Faire Ferien“ kann kostenfrei heruntergeladen werden:

https://www.misereor.de/informieren/fairer-handel/.

Filme für die Erde (red.): Die 2018 erschienene Publikation „Filme für die Erde“, die an der Leibnitz Universität Hannover erarbeitet wurde, bietet Unterrichtskonzepte und Materialien für den Einsatz von Dokumentarfilmen im Kontext einer Bildung für nachhaltige Entwicklung im Schulunterricht. Das Medium Film ist in außerordentlichem Maße dazu in der Lage - durch die Verbindung von Bildern, Klängen und Farben - Geschichten zu erzählen, die bewegen und in Erinnerung bleiben. Zur Verfügung stehen beispielsweise Materialien zu den Dokumentarfilmen „Unser täglich Brot“ und dem Umgang mit Tieren in der Lebensmittelindustrie, zu „Cowspiracy: The Sustainability Secret“ oder zu dem Film „The True Cost - Who Pays the Price for our Clothing?", der eine kritische Analyse der FastFashion-Industrie liefert. Die Publikation steht online zum Download bereit: https://www.didageo.uni-hannover.de/fileadmin/ institut/Filme_fuer_die_Erde_Webversion.pdf).

Globales Lernen mit Geflüchteten (red.): Geflüchtete werden häufig durch die gesetzlichen Regelungen für eine längere Zeitspanne zum Warten gezwungen und auf das Dasein als passive Hilfeempfangende reduziert. Deswegen initiierte das Bonner Netzwerk politik|atelier e. V. von 2015 bis 2017 zusammen mit dem Eine Welt Netz NRW und Engagement Global das Pilotprojekt „Geflüchtete als Bildungsbotschafter", wodurch die Schutzsuchenden in Veranstaltungen im Umfeld Globalen Lernens integriert wurden. Aus den gesammelten Erfahrungen ist die Publikation „Menschen in Bewegung. Leitfaden für Globales Lernen mit Geflüchteten“ entstanden, welche theoretische und praktische praktische Hinweise zur Einbindung von geflüchteten Personen in Referententätigkeiten gibt. Download kostenfrei möglich: https://eine-welt-netznrw.de/fileadmin/ewn/data/Material_fuer_Alle/BtE_GLmit_Gefluechteten.pdf zur Verfügung. 


\section{UNSERE BUCHEMPFEHLUNG}

vbw - Vereinigung der Bayerischen Wirtschaft e.V.

(Hrsg.)

\section{Bildung 2030 - veränderte Welt}

Fragen an die Bildungspolitik

2017, 232 Seiten, br., zweifarbig, $19,90 €$, ISBN 978-3-8309-3654-1

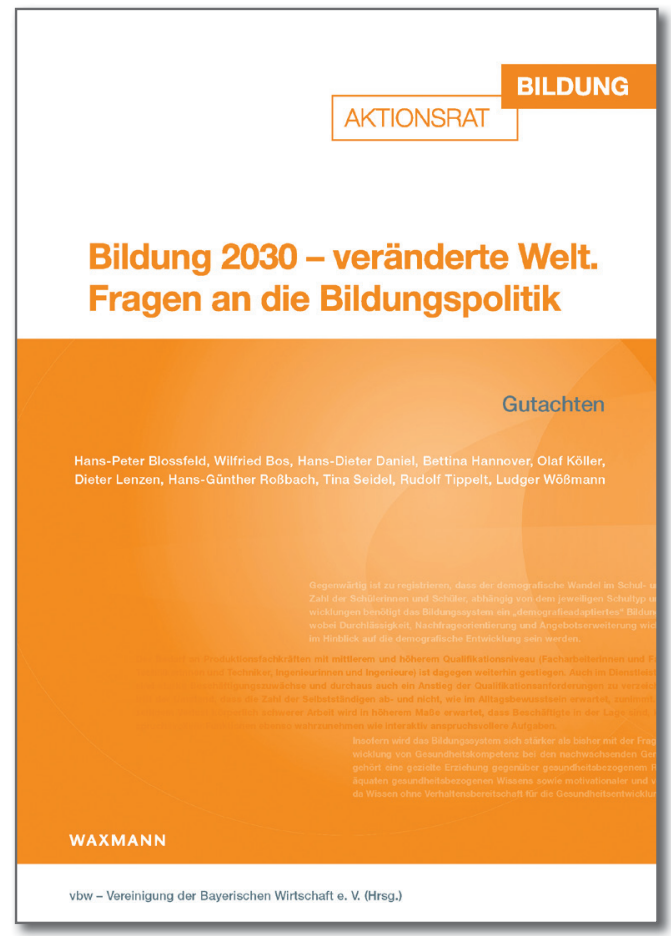

S eit der Veröffentlichung der ersten Studie im Jahr 2007 leistet der Ak-

$S$ tionsrat Bildung mit seinen Gutachten einen wichtigen Beitrag zur bildungspolitischen Diskussion und zu Reformprozessen innerhalb des deutschen Bildungssystems. Eine veränderte Welt, deren Herausforderungen vornehmlich aus dem raschen gesellschaftlichen, wirtschaftlichen, ökologischen und technologischen Wandel erwachsen, wird in der kommenden Dekade auch weitreichenden Einfluss auf die Rahmenbedingungen und Inhalte des Bildungssystems nehmen und entsprechende Anpassungen erfordern.

Im aktuellen Gutachten identifiziert der Aktionsrat Bildung zwölf bedeutende Wandlungs- und Entwicklungsprozesse in der deutschen Gesellschaft, die aus seiner Sicht für das Bildungssystem der Zukunft von zentraler Bedeutung sind. Die Beschreibung der Dynamik und Relevanz der einzelnen Bereiche für das Bildungssystem geht auf Kernfragen zu Curricula, Organisation, Struktur, Personal und Finanzen ein. Mit dem Gutachten hat sich der Aktionsrat Bildung das Ziel gesetzt, zu jedem Teilgebiet Leitfragen zu formulieren, von deren Beantwortung der Erfolg des deutschen Bildungswesens auch in Zukunft entscheidend abhängen wird. 